Universidade de Brasília

Instituto de Ciências Exatas

Departamento de Matemática

Uma Classe de Problemas Elípticos Assintoticamente Lineares em $\mathbb{R}^{N}$

$$
\text { por }
$$

Wesley de Freitas Mendes 


\title{
Uma Classe de Problemas Elípticos Assintoticamente Lineares em $\mathbb{R}^{N}$
}

por

\author{
Wesley de Freitas Mendes *
} Dissertação apresentada ao Departamento de Matemática da Universidade de Brasília, como parte dos
requisitos para obtenção do grau de

\section{MESTRE EM MATEMÁTICA}

Brasília, 01 de março de 2016

Comissão Examinadora:

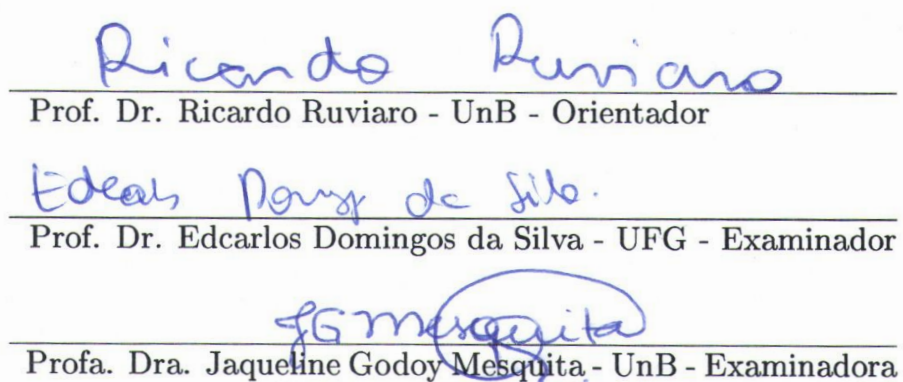

*O autor foi bolsista do CNPq durante a elaboração deste trabalho. 
Ficha catalográfica elaborada automaticamente, com os dados fornecidos pelo(a) autor(a)

Mendes, Wesley de Freitas

MM538c Uma Classe de Problemas Elípticos Assintoticamente Lineares em $\mathrm{R}^{\wedge} \mathrm{N}$. / Wesley de Freitas Mendes; orientador Ricardo Ruviaro. -- Brasília, 2016.

$77 \mathrm{p}$.

Dissertação (Mestrado - Mestrado em Matemática) -Universidade de Brasília, 2016.

1. Método variacional. 2. Passo da Montanha. 3. Problema elíptico. 4. Condição de Cerami. 5. Problema semilinear. I. Ruviaro, Ricardo, orient. II. Título. 
$O$ argumento mais convincente para explicar por que a cultura matemática dá tanta importância à prova de uma assertiva é o fato de que, ao contrário das outras ciências, temos o privilégio de poder fazê-lo. (A Música dos Números Primos) 


\section{Dedicatória}

Aos meus pais

José Carlos e Marli Maria 


\section{Agradecimentos}

Agradeço primeiramente a Deus, que me permitiu estar aqui e realizar meu sonho de conhecer esse mundo da Matemática. Por me mostrar como ser mais calmo e por revelar sua presença em minha vida. Agradeço por essa grande conquista.

Ao meu pai, José Carlos, por sempre estar presente em minha educação, pelos conselhos valiosos e pelo exemplo de homem que quero ser um dia. À minha mãe, Marli Maria, pelo amor incondicional e pelo carinho. À minha avó, Zelma, que para mim é sinônimo de amor. Agradeço pela paciência e pela confiança que depositaram em mim. Agradeço aos meus irmãos William, Wendel e Bárbara por iluminarem minha vida.

À minha namorada, Milene Soares, por estar sempre ao meu lado me motivando com um belo sorriso, por ser paciente e me aguentar falar de Matemática o tempo todo.

Aos meus melhores amigos, José Maria e Rony Lins, que fizeram de minha vida uma festa. À minha colega Mayra Soares, que esteve comigo desde o início do Mestrado me animando com seu jeito único de ser. Agradeço aos demais colegas de curso que fizeram parte dessa jornada, a eles meu sincero obrigado.

Aos professores do Departamento, agradeço pelos conhecimentos transmitidos e pelo tempo disponibilizado. Em especial agradeço à professora Liliane de Almeida, ao professor Mauro Rabelo e à professora Cátia Regina. Agradeço também à professora Jaqueline Godoy e ao professor Edcarlos Domingos por formarem minha banca examinadora.

Ao meu orientador Ricardo Ruviaro, agradeço pelos ensinamentos valiosos e por me colocar no caminho certo sempre que me desviava. Serei eternamente grato por ter me acolhido como orientando. Agradeço por ser esse exemplo de profissional dedicado e mais do que um orientador sinto que ganhei um amigo.

Agradeço à $C N P q$ pelo apoio financeiro à este trabalho. 


\section{Resumo}

Buscaremos neste trabalho estabelecer a existência de solução positiva para o problema semilinear

$$
\left(P_{\lambda}\right) \quad-\Delta u+\lambda u=f(x, u) u, \quad x \in \mathbb{R}^{N},
$$

onde $\lambda>0$ é um parâmetro e $f \in C\left(\mathbb{R}^{N} \times \mathbb{R}^{+}, \mathbb{R}^{+}\right)$satisfaz algumas hipóteses específicas. Para isso, usamos a técnica variacional e nossa principal ferramenta será o Teorema do Passo da Montanha com condição de Cerami. Estabeleceremos também resultados de multiplicidade para o problema $\left(P_{\lambda}\right)$ com uma condição extra de simetria na não linearidade.

Palavras-Chave: Problema Semilinear; Solução Positiva; Passo da Montanha; Condição de Cerami; Técnica Variacional; Resultados de Multiplicidade. 


\section{Abstract}

We seek in this work to establish the existence of positive solutions for the semilinear problem

$$
\left(P_{\lambda}\right) \quad-\Delta u+\lambda u=f(x, u) u, \quad x \in \mathbb{R}^{N}
$$

where $\lambda>0$ is a parameter and $f \in C\left(\mathbb{R}^{N} \times \mathbb{R}^{+}, \mathbb{R}^{+}\right)$satisfies some specifics hypotheses. For this, we use the variational technique and our main tool will be the Mountain-Pass Theorem with Cerami condition. We establish, as well, multiplicity results for the problem $\left(P_{\lambda}\right)$ with an extra symmetry condition on the nonlinearity.

Key-Words: Semilinear Problem; Positive Solution; Mountain-Pass; Cerami Condition; Variational Technique; Multiplicity Results. 


\section{Notações}

Ao longo deste trabalho, vamos utilizar as seguintes notações:

$B_{R}, B_{R}(0)$

$B_{R}(y), B_{R}+y$,

$p^{*}=\frac{N p}{N-p}$,

$(P S)_{c}$

$(C e)_{c}$,

$u_{n} \rightarrow u$,

$u_{n} \rightarrow u$,

supp

$\langle\cdot, \cdot\rangle$,

$C, C_{i}$,

$o(1)$

$\mathbb{R}^{+}$

$D(A)$,

$\sigma(A)$

$\delta_{i j}=\left\{\begin{array}{l}1, \text { se } i=j \\ 0, \text { se } i \neq j\end{array}\right.$, bola aberta centrada em zero e com raio $R$.

bola centrada em $y$ e com raio $R$.

expoente crítico de Sobolev.

condição de Palais-Smale no nível $c$.

condição de Cerami no nível $c$.

convergência forte (em norma).

convergência fraca.

suporte da função $f$.

produto interno.

denotam constantes positivas.

ordem pequena.

conjunto dos números reais não negativos.

domínio do operador $A$.

espectro do operador $A$.

delta de Kronecker. 


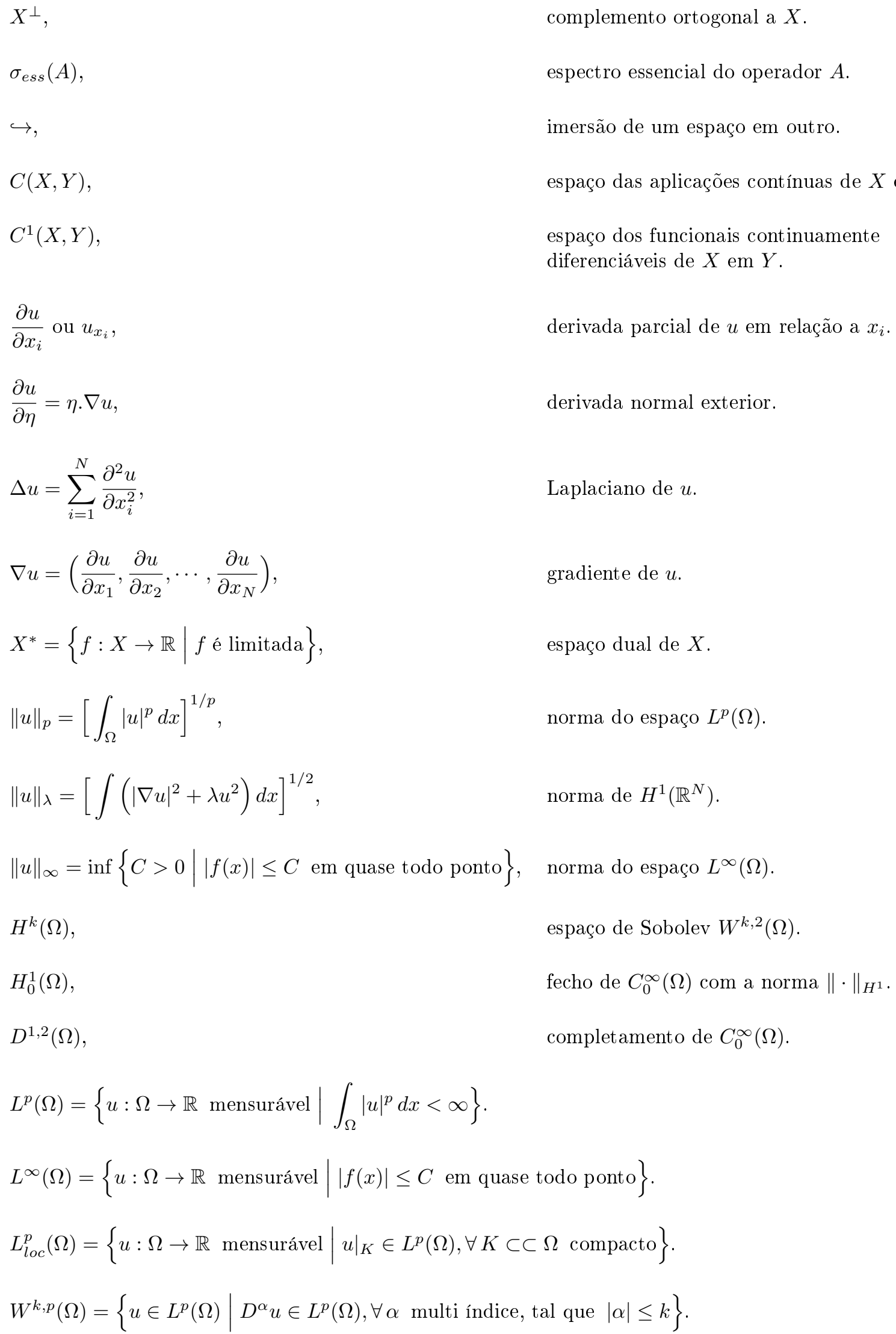




\section{Sumário}

1 Preliminares $\quad 3$

1.1 Operadores Lineares de Segunda Ordem . . . . . . . . . . . . . . . . . 3

1.2 Análise Funcional . . . . . . . . . . . . . . . . . . . . . . . . . . . . . . . . . 11

1.3 Teoria da Medida . . . . . . . . . . . . . . . . . . . . . . 13

1.4 Espaços de Sobolev . . . . . . . . . . . . . . . . . . . . . . 18

1.5 Caracterização de $\lambda_{1} \ldots \ldots \ldots \ldots \ldots \ldots \ldots \ldots \ldots$

2 Estrutura Variacional - A Condição de Cerami 23

2.1 Condições para $f \ldots \ldots \ldots \ldots \ldots \ldots \ldots \ldots \ldots$

2.2 Regularidade do Funcional $I_{\lambda} \ldots \ldots \ldots \ldots \ldots \ldots \ldots$. . . . . . . . . . . . . 27

2.3 Condição de Cerami . . . . . . . . . . . . . . . . . . . . . . . . 30

3 Existência de Solução Positiva $\quad 56$

3.1 Geometria do Passo da Montanha . . . . . . . . . . . . . . . . . . . 56

3.2 Existência de Solução Positiva . . . . . . . . . . . . . . . . . . . . . . . . 61

3.3 Exemplo de $f \ldots \ldots \ldots \ldots \ldots \ldots \ldots \ldots \ldots$

4 Existência de Múltiplas Soluções $\quad 68$

4.1 Simetria na não linearidade . . . . . . . . . . . . . . . . . . . . . . . . . 68

4.2 Resultado de Multiplicidade . . . . . . . . . . . . . . . . . . . . 70

$\begin{array}{lr}\text { Referências Bibliográficas } & \mathbf{7 6}\end{array}$ 


\section{Introdução}

Estudaremos neste trabalho a questão da existência de solução positiva devido a Costa e Tehrani [6], bem como resultados de multiplicidade do problema semilinear:

$$
\left(P_{\lambda}\right) \quad-\Delta u+\lambda u=f(x, u) u, \quad x \in \mathbb{R}^{N},
$$

onde $\lambda>0$ é um parâmetro e $f \in C\left(\mathbb{R}^{N} \times \mathbb{R}^{+}, \mathbb{R}^{+}\right)$satisfaz as seguintes condições (condições precisas serão indicadas no Capítulo 2):

$$
\lim _{s \rightarrow 0} f(x, s)=0, \quad \text { uniformemente em } x
$$

$f(x, s)$ é uma função não decrescente de $s \in[0, \infty)$, para todo $x \in \mathbb{R}^{N}$, e existem funções $g \in C\left(\mathbb{R}^{N}, \mathbb{R}^{+}\right)$ e $h \in C\left(\mathbb{R}^{+}, \mathbb{R}^{+}\right)$com:

$$
\lim _{s \rightarrow \infty} f(x, s)=g(x), \quad \lim _{|x| \rightarrow \infty} f(x, s)=h(s) \quad \text { e } \quad \lim _{|x| \rightarrow \infty, s \rightarrow \infty} f(x, s)=l_{\infty} \in(0, \infty)
$$

Nessas condições, $\left(P_{\lambda}\right)$ é um problema assintoticamente linear. Quando essa equação é considerada em um domínio limitado $\Omega \subset \mathbb{R}^{N}$ (com, digamos, a condição de fronteira de Dirichlet), existe uma extensa literatura que aborda existência de solução, bem como resultados de multiplicidade. De particular interesse é então o caso ressonante, onde $-\lambda \in \sigma(S)$ e $S$ é a linearização assintótica do problema. Em outras palavras, $S: D(S) \subset L^{2}(\Omega) \rightarrow L^{2}(\Omega)$ é o operador dado por:

$$
S u(x)=-\Delta u(x)-g(x) u(x) \quad \text { e } \quad D(S)=H_{0}^{1}(\Omega) \cap H^{2}(\Omega) .
$$

Nesse caso, a questão de existência de soluções é mais delicada. É claro que desde que $\Omega$ é limitado, $\sigma(S)$ consiste em um conjunto enumerável de autovalores com multiplicidades finitas e, portanto, ressonância é um fenômeno raro.

Por outro lado, para o nosso conhecimento, menos se tem feito quando $\Omega=\mathbb{R}^{N}$ no caso do problema $\left(P_{\lambda}\right)$. Uma das dificuldades nesse caso é o fato de que o espectro do operador $S$ inclui uma parte essencial, a saber $\left[-l_{\infty}, \infty\right)$, de modo que precisamos lidar com um problema ressonante muito mais complicado. A outra dificuldade em lidar com tais problemas em $\mathbb{R}^{N}$ é a falta de compacidade exibida pelo funcional energia correspondente, digamos, como medido pela conhecida condição de Palais-Smale. A seguir, descreveremos como este trabalho será apresentado. 
No Capítulo 1, apresentamos conceitos preliminares essenciais ao bom desenvolvimento dos nossos principais teoremas e lemas. No Capítulo 2, introduzimos a estrutura variacional e estudamos a condição de compacidade de Cerami. Primeiro, definiremos

$$
\Lambda=\inf \left\{\int\left[|\nabla u|^{2}-g(x) u^{2}\right] d x \mid u \in H^{1}\left(\mathbb{R}^{\mathbb{N}}\right), \int u^{2} d x=1\right\} .
$$

Se $\left(P_{\lambda}\right)$ possui uma solução então, necessariamente, devemos ter $\lambda<|\Lambda|$, de forma que assumimos $0<\lambda<|\Lambda|$ ao longo do trabalho. Ademais, explorando os resultados para problemas lineares de autovalor em $\mathbb{R}^{N}$, e sistematicamente usando o método de Concentração e Compacidade de Lions, somos capazes de mostrar que a compacidade de Cerami vale para um certo intervalo de valores de energia do funcional correspondente.

No Capítulo 3, provamos nosso principal resultado de existência e estabelecemos a existência de uma solução positiva de $\left(P_{\lambda}\right)$, para todo $0<\lambda<|\Lambda|$. Isto é feito primeiramente achando uma candidata para um nível crítico através do Teorema do Passo da Montanha. Então, sob uma condição adicional para $f(x, s)$, um argumento de comparação com o problema no infinito é usado para mostrar que nosso nível candidato é de fato o nível onde a compacidade de Cerami vale, permitindo assim a aplicação de teoremas de pontos críticos. Vale notar que, como $\Lambda$ é o menor ponto espectral de $S$ e $\sigma_{\text {ess }}(S)=\left[-l_{\infty}, \infty\right)$, temos $\Lambda \leq-l_{\infty}$ e então, se $0<\lambda<|\Lambda|$, pode muito bem acontecer que $-\lambda \in \sigma(S)$. Não obstante, nosso resultado de existência, que veremos no Teorema 3.1, é independente do problema ser ou não ressonante.

Finalmente, no Capítulo 4, consideramos a questão de existência de múltiplas soluções quando $f(x, s)$ é uma função par de $s$. Nossa principal ferramenta nessa seção é uma variante do teorema do ponto crítico abstrato para funcionais pares.

Salvo menção em contrário, todas as integrais serão tomadas sobre todo o $\mathbb{R}^{N}$ e $C, C_{i}$ representarão constantes positivas. 


\section{Capítulo}

\section{Preliminares}

Antes de começarmos nosso trabalho principal, precisamos conhecer alguns resultados que serão úteis no decorrer do mesmo.

\subsection{Operadores Lineares de Segunda Ordem}

Vamos conhecer primeiramente algumas propriedades de operadores lineares de $2^{\mathrm{a}}$ ordem, o lema de Hopf e o lema de Lions, que serão usados no decorrer do trabalho. No que segue, estamos nos baseando em Evans [8] e Adams [1].

Considere o operador linear de $2^{\text {a }}$ ordem dado pela expressão

$$
L u:=\sum_{i, j=1}^{N} a^{i j}(x) u_{x_{i} x_{j}}+\sum_{i=1}^{N} b^{i}(x) u_{x_{i}}+c(x) u
$$

onde $u \in C^{2}(\Omega)$, os coeficientes $a^{i j}, b^{i}, c: \Omega \rightarrow \mathbb{R}$ são funções dadas e $\Omega \subset \mathbb{R}^{N}$ é um aberto limitado. Observe que como $u \in C^{2}(\Omega)$, então pelo Teorema de Schwarz temos $u_{x_{i} x_{j}}=u_{x_{j} x_{i}}$, para todo $i, j \in$ $1, \ldots, N$. Logo, podemos supor que, para cada $x \in \Omega$, a matriz $A(x)=\left[a^{i j}(x)\right]_{N \times N}$ é simétrica.

Definição 1.1. Dizemos que o operador definido em (1.1) é elíptico no ponto $x \in \Omega$ se a forma quadrática associada à matriz $A(x)$ é positiva definida, ou seja, se $\lambda(x)$ for o menor autovalor de $A(x)$, então

$$
\sum_{i, j=1}^{N} a^{i j}(x) \xi_{i} \xi_{j} \geq \lambda(x)|\xi|^{2}>0
$$

para todo $\xi=\left(\xi_{1}, \ldots, \xi_{N}\right) \in \mathbb{R}^{N} \backslash\{0\}$. O operador é dito eliptico em $\Omega$ se for eliptico em cada ponto de $\Omega$. Finalmente, dizemos que $L$ é uniformemente eliptico em $\Omega$ se existe $\theta_{0}>0$ tal que $\lambda(x) \geq \theta_{0}$ para todo $x \in \Omega$. Dizemos que L está na forma divergente quando

$$
L u:=-\sum_{i, j}^{N}\left(a^{i j}(x) u_{x_{i}}\right)_{x_{j}}+\sum_{i=1}^{N} b^{i}(x) u_{x_{i}}+c(x) u
$$


Observe que quando $L$ é uniformemente elíptico, vale a desigualdade

$$
\xi A(x) \xi=\sum_{i, j=1}^{N} a^{i j}(x) \xi_{i} \xi_{j} \geq \theta_{0}|\xi|^{2}, \quad \xi \in \mathbb{R}^{N}
$$

E assim tomando $\xi=e_{i}$, vetor da base canônica de $\mathbb{R}^{N}$, obtemos

$$
e_{i} A(x) e_{i}=a^{i i}(x) \geq \theta_{0}\left|e_{i}\right|^{2}=\theta_{0}, \quad i=1, \ldots, N \text { e } x \in \Omega
$$

Teorema 1.1. Seja $L$ um operador uniformemente eliptico em $\Omega$ com c $\equiv 0$ em $\Omega$. Se $u \in C^{2}(\Omega) \cap C(\bar{\Omega})$ e $L u \geq 0$ em $\Omega$, então $\max _{\bar{\Omega}} u=\max _{\partial \Omega} u$.

Demonstração. Suponha inicialmente que $L u>0$ em $\Omega$ e que existe $\tilde{x} \in \Omega$ tal que $u(\tilde{x})=\max _{\bar{\Omega}} u$. Como $L$ é uniformemente elíptico, a matriz dos coeficientes $A=A(\tilde{x})$ é positiva definida. Por $A$ ser simétrica, existe uma matriz ortogonal $O=O_{N \times N}$, ou seja, $O^{-1}=O^{T}$, tal que

$$
O A O^{T}=\left[\begin{array}{cccc}
\lambda_{1} & 0 & \ldots & 0 \\
0 & \lambda_{2} & \cdots & 0 \\
: & : & \ddots & 0 \\
0 & 0 & \ldots & \lambda_{N}
\end{array}\right]
$$

e por $L$ ser uniformemente elíptico, temos $\lambda_{i} \geq \theta_{0}>0, i=1, \ldots, N$. O termo geral da matriz acima é dado por

$$
\delta_{k l} \lambda_{k}=\sum_{j=1}^{N} o_{k j} \sum_{i=1}^{N} a^{i j} o_{i l}^{T}=\sum_{i, j=1}^{N} o_{k j} a^{i j} o_{l i} .
$$

Considerando agora a nova variável $y(x):=\tilde{x}+O(x-\tilde{x})$, note que $y(\tilde{x})=\tilde{x}$ e

$$
\begin{aligned}
y-\tilde{x} & =O(x-\tilde{x}) & \Rightarrow \\
O^{T}(y-\tilde{x})=O^{T} O(x-\tilde{x}) & =O^{-1} O(x-\tilde{x})=x-\tilde{x} & \Rightarrow \\
\tilde{x}+O^{T}(y-\tilde{x}) & =x &
\end{aligned}
$$

assim

$$
u(x)=u\left(\tilde{x}+O^{T}(y-\tilde{x})\right):=v(y(x)) .
$$

Observe que $y(\tilde{x})$ é o ponto de máximo da função $v$, pois $\tilde{x}$ é ponto de máximo de $u$, e portanto

$$
\nabla u(\tilde{x})=\nabla v(y(\tilde{x}))=\nabla v(\tilde{x})=0 \quad \text { e } \quad D^{2} v(\tilde{x}) \leq 0
$$

com a segunda inequação acima significando que a matriz Hessiana do $v$ no ponto $\tilde{x}$ é não positiva. Se $y=\left(y_{1}, \ldots, y_{N}\right)$, então

$$
y_{k}=\tilde{x}_{k}+\sum_{j=1}^{N} o_{k j}\left(x_{j}-\tilde{x}_{j}\right) \Rightarrow \frac{\partial y_{k}}{\partial x_{i}}=o_{k i}
$$


para cada $k=1, \ldots, N$, logo

$$
u_{x_{i}}=\sum_{k=1}^{N} \frac{\partial v}{\partial y_{k}} \frac{\partial y_{k}}{\partial x_{i}}=\sum_{k=1}^{N} v_{y_{k}} o_{k i},
$$

e do mesmo modo

$$
u_{x_{i} x_{j}}=\sum_{k, l=1}^{N} v_{y_{k} y_{l} o_{k i} o_{l j}},
$$

para $i, j=1, \cdots, N$.

Como $\nabla u(\tilde{x})=0$, obtemos

$$
\begin{array}{rlrl}
L u(\tilde{x}) & =\sum_{i, j=1}^{N} a^{i j}(\tilde{x}) u_{x_{i} x_{j}}(\tilde{x})+\sum_{i=1}^{N} b^{i}(\tilde{x}) u_{x_{i}}(\tilde{x}), & & \text { pois } c \equiv 0, \text { em } \Omega \\
& =\sum_{i, j=1}^{N} a^{i j}(\tilde{x}) u_{x_{i} x_{j}}(\tilde{x}), & & \text { pois } \sum_{i=1}^{N} b^{i}(\tilde{x}) u_{x_{i}}(\tilde{x})=(b . \nabla u)(\tilde{x})=0 \\
& =\sum_{i, j=1}^{N} a^{i j}(\tilde{x}) \sum_{k, l=1}^{N} v_{y_{k} y_{l} o_{k i} o_{l j},} & & \text { por }(1.4) \\
& =\sum_{k, l=1}^{N} v_{y_{k} y_{l}} \sum_{i, j=1}^{N} a^{i j}(\tilde{x}) o_{k i} o_{l j} & & \\
& =\sum_{k, l=1}^{N} v_{y_{k} y_{l}} \delta_{k l} \lambda_{k}, & & \text { por }(1.3) \\
& =\sum_{k=1}^{N} v_{y_{k} y_{k}} \lambda_{k} .
\end{array}
$$

Uma vez que $D^{2} v(\tilde{x}) \leq 0$, temos que $e_{k} D^{2} v(\tilde{x}) e_{k} \leq 0$ e isto implica que $v_{y_{k} y_{k}}(\tilde{x}) \leq 0$, para $k=1, \ldots, N$. Como os números $\lambda_{i}{ }^{\prime} s$ são positivos, concluímos da expressão acima que

$$
L u(\tilde{x})=\sum_{k=1}^{N} v_{y_{k} y_{k}}(\tilde{x}) \lambda_{k} \leq 0,
$$

o que é um absurdo. Logo, se $L u>0 \mathrm{em} \Omega$, a função $u$ não pode assumir seu máximo em $\Omega$, isto é, $\max _{\bar{\Omega}} u=\max _{\partial \Omega} u$.

Consideremos agora o caso geral $L u \geq 0$. Seja $\gamma \in \mathbb{R}$ arbitrário, $\varepsilon>0$ e considere

$$
u_{\varepsilon}(x):=u(x)+\varepsilon e^{\gamma x_{1}}, \quad x=\left(x_{1}, \ldots, x_{N}\right) \in \Omega .
$$

Usando a definição de $L$, a equação (1.2), a regularidade dos coeficientes e $L u \geq 0$, obtemos

$$
\begin{aligned}
L u_{\varepsilon} & =L u+\varepsilon L\left(e^{\gamma x_{1}}\right) \\
& =L u+\varepsilon e^{\gamma x_{1}}\left(a^{11}(x) \gamma^{2}+b^{1}(x) \gamma\right) \\
& \geq \varepsilon e^{\gamma x_{1}}\left(\theta_{0} \gamma^{2}-\left\|b^{1}\right\|_{\infty} \gamma\right) .
\end{aligned}
$$

Escolhendo $\gamma>0$ suficientemente grande de modo que $L u_{\varepsilon}>0$, podemos usar a primeira parte da 
demonstração para concluir que

$$
\max _{\bar{\Omega}} u_{\varepsilon}=\max _{\partial \Omega} u_{\varepsilon}
$$

Mas $u \leq u_{\varepsilon}$, e portanto

$$
\max _{\bar{\Omega}} u \leq \max _{\bar{\Omega}} u_{\varepsilon}=\max _{\partial \Omega} u_{\varepsilon} \leq \max _{\partial \Omega} u+\varepsilon \max _{\partial \Omega} e^{\gamma x_{1}}
$$

Fazendo $\varepsilon \rightarrow 0^{+}$, concluímos que $\max _{\bar{\Omega}} u \leq \max _{\partial \Omega} u$. Uma vez que a desigualdade contrária é trivialmente satisfeita, concluímos que

$$
\max _{\bar{\Omega}} u=\max _{\partial \Omega} u
$$

Teorema 1.2. (Princípio do Máximo Fraco) Seja L um operador uniformemente elíptico em $\Omega$ com $c \leq 0$ em $\Omega$. Se $u \in C^{2}(\Omega) \cap C(\bar{\Omega})$ e Lu $\geq 0$ em $\Omega$, então $\max _{\bar{\Omega}} u \leq \max _{\partial \Omega} u^{+}$.

Demonstração. Seja $\Omega^{+}:=\{x \in \Omega \mid u(x)>0\}$. Se $\Omega^{+}$for vazio, então $u \leq 0$ em $\Omega$. Tome $x \in \partial \Omega$ e $\left(x_{n}\right) \subset \Omega$ tal que $x_{n} \rightarrow x$. Pela continuidade de $u$ até a fronteira, temos que $u(x)=\lim _{n \rightarrow \infty} u\left(x_{n}\right) \leq 0$, $\operatorname{assim} u \leq 0$ em $\bar{\Omega}$ e portanto

$$
\max _{\bar{\Omega}} u \leq 0=\max _{\partial \Omega} u^{+}
$$

onde $u^{+}(x):=\max \{u(x), 0\}$.

Logo, podemos supor que $\Omega^{+} \neq \varnothing$. Tome então $x \in \Omega^{+}$, logo $u(x)>0$ e como $u$ é contínua, então existe $r>0$ tal que $u>0$ em $B_{r}(x)$ e assim $\Omega^{+}$é aberto em $\Omega$ e, portanto, aberto em $\mathbb{R}^{N}$. Seja

$$
K u:=L u-c(x) u=\sum_{i, j=1}^{N} a^{i j}(x) u_{x_{i} x_{j}}+\sum_{i=1}^{N} b^{i}(x) u_{x_{i}},
$$

desse modo, como $c \leq 0$ em $\Omega$, obtemos $K u \geq 0$, para $u \in C^{2}\left(\Omega^{+}\right) \cap C\left(\overline{\Omega^{+}}\right)$. Segue então do Teorema 1.1 , aplicado ao operador $K$, que

$$
\max _{\overline{\Omega^{+}}} u=\max _{\partial \Omega^{+}} u .
$$

Uma vez que $\bar{\Omega}=\overline{\Omega^{+}} \cap \overline{\Omega \backslash \Omega^{+}}$e $u \leq 0$ em $\overline{\Omega \backslash \Omega^{+}}$, segue que

$$
\max _{\bar{\Omega}} u=\max _{\overline{\Omega^{+}}} u=\max _{\partial \Omega^{+}} u
$$

Assim, é suficiente mostrar que

$$
\max _{\partial \Omega^{+}} u \leq \max _{\partial \Omega} u^{+} .
$$

Considere $x_{0} \in \partial \Omega^{+}$tal que $u\left(x_{0}\right)=\max _{\partial \Omega^{+}} u$. A continuidade de $u$ e a definição de $\Omega^{+}$implicam que $u\left(x_{0}\right) \geq 0$. Temos dois casos a considerar:

Caso 1) $u\left(x_{0}\right)=0$ :

Neste caso devemos ter $u \leq 0$ em $\bar{\Omega}$ pois $u(x) \leq \max _{\bar{\Omega}} u=\max _{\partial \Omega^{+}} u=u\left(x_{0}\right)=0$. Logo, $u^{+}=0$ em $\partial \Omega \mathrm{e}$ portanto

$$
u\left(x_{0}\right)=\max _{\partial \Omega^{+}} u=0=\max _{\partial \Omega} u^{+}
$$


Caso 2) $u\left(x_{0}\right)>0$ :

Neste caso, como $\Omega^{+}$é aberto em $\Omega$, devemos ter $x_{0} \in \partial \Omega$. De fato, se não fosse assim, teríamos $x_{0} \in \Omega$ e como $u$ é contínua, então $u$ seria positiva em toda uma bola $B_{\varepsilon}\left(x_{0}\right) \subset \Omega^{+}$, contrariando o fato de que $x_{0} \in \partial \Omega^{+}$. Daí

$$
\max _{\partial \Omega^{+}} u=u\left(x_{0}\right)=u^{+}\left(x_{0}\right) \leq \max _{\partial \Omega} u^{+},
$$

e temos o resultado.

Teorema 1.3. (Princípio da Comparação). Seja L um operador uniformemente elíptico em $\Omega$ com $c \leq 0$ e $u \in C^{2}(\Omega) \cap C(\bar{\Omega})$. Se $L u \geq 0$ em $\Omega$ e $u \leq 0$ em $\partial \Omega$, então $u \leq 0$ em $\bar{\Omega}$.

Demonstração. Pelo Teorema 1.2, temos que

$$
u(x) \leq \max _{\bar{\Omega}} u \leq \max _{\partial \Omega} u^{+}=0,
$$

pois $u^{+}=0$ em $\partial \Omega, \operatorname{logo} u \leq 0$ em $\bar{\Omega}$.

Lema 1.1. (Lema de Hopf). Suponha que $B \subset \mathbb{R}^{N}$ é uma bola aberta, $L$ é um operador uniformemente elíptico em $B, u \in C^{2}(B)$ e $L u \geq 0$ em $B$. Suponha ainda que existe $x_{0} \in \partial B$ tal que u é contínua em $x_{0}$ e $u(x)<u\left(x_{0}\right)$, para todo $x \in B$. Então,

i) se $c=0$ em $B$ e existe a derivada normal $\frac{\partial u}{\partial \eta}\left(x_{0}\right)$, então $\frac{\partial u}{\partial \eta}\left(x_{0}\right)>0$;

ii) se $c \leq 0$ em $\Omega$ e $u\left(x_{0}\right) \geq 0$, então vale o mesmo resultado do item acima.

Antes de provar o lema de Hopf vale observar que se $x_{0} \in \partial B$ é um ponto de máximo local e existe $\frac{\partial u}{\partial \eta}\left(x_{0}\right)$, então é sempre verdade que

$$
\frac{\partial u}{\partial \eta}\left(x_{0}\right)=\lim _{h \rightarrow 0^{-}} \frac{u\left(x_{0}+h \eta\right)-u\left(x_{0}\right)}{h} \geq 0
$$

independente do sinal de $L u$. A informação adicional dada pelo lema é que a desigualdade é estrita.

Demonstração. Podemos supor, sem perda de generalidade, que $u \in C(\bar{B})$ e que $u(x)<u\left(x_{0}\right)$ para todo $x \in \bar{B} \backslash\left\{x_{0}\right\}$. De fato, se não for esse o caso, é suficiente tomar uma nova bola $B^{\prime} \subset B$ que é internamente tangente à $B$ no ponto $x_{0}$. Além disso, conforme veremos posteriormente, podemos também supor que $B=B_{r}(0)$.

Feitas as considerações acima, vamos assumir inicialmente as hipóteses do item (ii) e considerar, para $\gamma>0$ a ser determinado, a função

$$
v(x):=e^{-\gamma|x|^{2}}-e^{-\gamma r^{2}}, \quad x \in B .
$$

Para cada $i, j=1, \ldots, N$, temos que

$$
v_{x_{i}}=-2 \gamma x_{i} e^{-\gamma|x|^{2}}
$$

e

$$
v_{x_{i} x_{j}}= \begin{cases}4 \gamma^{2} x_{i} x_{j} e^{-\gamma|x|^{2}}, & \text { se } i \neq j, \\ 4 \gamma^{2} x_{i}^{2} e^{-\gamma|x|^{2}}-2 \gamma e^{-\gamma|x|^{2}}, & \text { se } i=j\end{cases}
$$


ou seja,

$$
v_{x_{i} x_{j}}=\left(4 \gamma^{2} x_{i} x_{j}-2 \gamma \delta_{i j}\right) e^{-\gamma|x|^{2}}
$$

de modo que

$$
\begin{aligned}
L v(x) & =\sum_{i, j=1}^{N} a^{i j}(x) v_{x_{i} x_{j}}+\sum_{i=1}^{N} b^{i}(x) v_{x_{i}}+c(x) v \\
& =e^{-\gamma|x|^{2}}\left(\sum_{i, j=1}^{N}\left(4 \gamma^{2} a^{i j}(x) x_{i} x_{j}-2 \gamma \delta_{i j} a^{i j}(x)\right)-2 \gamma \sum_{i=1}^{N}\left(b^{i}(x) x_{i}\right)+c(x)\right)-c(x) e^{-\gamma r^{2}}
\end{aligned}
$$

Usando as hipótese sobre os coeficientes de $L$, temos que

$$
\begin{gathered}
\sum_{i, j=1}^{N} a^{i j}(x) x_{i} x_{j} \geq \theta_{0}|x|^{2}, \quad \sum_{i=1}^{N} b^{i}(x) x_{i} \leq|x| \sum_{i=1}^{N}\left\|b^{i}\right\|_{\infty}=C_{1}, \\
\sum_{i, j=1}^{N} \delta_{i j} a^{i j}(x) \leq \sum_{i=1}^{N}\left\|a^{i j}\right\|_{\infty}=C_{2}
\end{gathered}
$$

e

$$
-c(x) e^{-\gamma r^{2}} \geq 0, \quad \text { pois } \quad c \leq 0
$$

com $C_{1}, C_{2} \geq 0$. As estimativas acima implicam que

$$
L v(x) \geq e^{-\gamma|x|^{2}}\left(4 \gamma^{2} \theta_{0}|x|^{2}-2 \gamma\left(C_{1}+C_{2}\right)-\|c\|_{\infty}\right) .
$$

Desse modo, fazendo $C_{3}:=C_{1}+C_{2}$ e denotando $A_{r}:=B_{r}(0) \backslash B_{r / 2}(0)$, temos que, para todo $x \in A_{r}$, vale

$$
L v(x) \geq e^{-\gamma|x|^{2}}\left(4 \gamma^{2} \theta_{0}\left(\frac{r}{2}\right)^{2}-2 \gamma C_{3}-\|c\|_{\infty}\right) .
$$

Escolhendo $\gamma>0$ grande o suficiente de modo que o termo entre parênteses acima seja positivo, concluímos que

$$
L v \geq 0, \text { em } A_{r}
$$

Note que se $x \in B=B_{r}(0)$, então

$$
\begin{aligned}
|x|^{2} & <r^{2} & \Rightarrow \\
-\gamma|x|^{2} & >-\gamma r^{2} & \Rightarrow \\
e^{-\gamma|x|^{2}} & >e^{-\gamma r^{2}}, &
\end{aligned}
$$

logo $v(x)=e^{-\gamma|x|^{2}}-e^{-\gamma r^{2}}>0$ em $B$ e, em particular, $v$ é positiva em $\partial B_{r / 2}(0)$ e uma vez que $x_{0}$ é um ponto de máximo estrito de $u$ e a função $v$ é contínua no compacto $\partial B_{r / 2}(0)$, podemos escolher $\varepsilon>0$ de tal modo que

$$
u\left(x_{0}\right) \geq u(x)+\varepsilon v(x), \quad x \in \partial B_{r / 2}(0)
$$

Note ainda que a desigualdade acima permanece válida em $\partial B_{r}(0)$ pois, nesse conjunto a função $v$ se anula. Desse modo, a função

$$
w(x)=u(x)+\varepsilon v(x)-u\left(x_{0}\right)
$$


é tal que

$$
\begin{cases}L w=L u+\varepsilon L v-c(x) u\left(x_{0}\right) \geq 0, & \text { em } A_{r}, \\ w \leq 0, & \text { em } \partial A_{r} .\end{cases}
$$

Segue então do Princípio da Comparação (Teorema 1.3) que $w \leq 0$ em $\overline{A_{r}}$. Observe agora que, como $x_{0} \in \partial B$, temos que $v\left(x_{0}\right)=0$. Logo, $w\left(x_{0}\right)=u\left(x_{0}\right)+\varepsilon v\left(x_{0}\right)-u\left(x_{0}\right)=0$ e, portanto, $x_{0}$ é um ponto de máximo de $w$ em $\bar{A}_{r}$. Desse modo, pela observação antes da demonstração, supondo que existe a derivada normal de $u$ no ponto $x_{0}$, devemos ter $\frac{\partial w}{\partial \eta}\left(x_{0}\right) \geq 0$, o que implica em

$$
\begin{aligned}
\frac{\partial w}{\partial \eta}\left(x_{0}\right) & =\nabla w\left(x_{0}\right) \cdot \eta \\
& =\nabla\left(u+\varepsilon v-u\left(x_{0}\right)\right)\left(x_{0}\right) \cdot \eta \\
& =\nabla u\left(x_{0}\right) \cdot \eta+\varepsilon \nabla v\left(x_{0}\right) \cdot \eta \geq 0,
\end{aligned}
$$

logo, notando que $\frac{x_{0}}{r}$ é o vetor normal unitário de $B_{r}(0)$, temos

$$
\begin{aligned}
\nabla u\left(x_{0}\right) \cdot \eta & =\frac{\partial u}{\partial \eta}\left(x_{0}\right) \\
& \geq-\varepsilon \nabla v\left(x_{0}\right) \cdot\left(\frac{x_{0}}{r}\right) \\
& =-\varepsilon\left(-2 \gamma x_{0} e^{-\gamma\left|x_{0}\right|^{2}}\right)\left(\frac{x_{0}}{r}\right) \\
& =2 \gamma \varepsilon \frac{\left|x_{0}\right|^{2}}{r} e^{-\gamma\left|x_{0}\right|^{2}}>0 .
\end{aligned}
$$

Isso estabelece a veracidade de (ii) no caso em que a bola $B$ está centrada na origem. Para o caso geral em que $B=B_{r}(y)$, basta considerar $v(x)=e^{-\gamma|x-y|^{2}}-e^{-\gamma r^{2}}$, para $x \in B_{r}(y)$ e proceder como acima. A prova do item (i) também pode ser feita repetindo os mesmos passos.

Precisamos também conhecer o famoso Lema de Lions.

Lema 1.2. (Lema de Lions) Sejam $R>0$ e $2 \leq q<2^{*}$. Se $\left(u_{n}\right)$ é limitada em $H^{1}\left(\mathbb{R}^{N}\right)$ e se

$$
\sup _{y \in \mathbb{R}^{N}} \int_{B_{R}(y)}\left|u_{n}\right|^{q} d x \rightarrow 0, \quad \text { quando } n \rightarrow+\infty
$$

então

$$
u_{n} \rightarrow 0 \text { em } L^{p}\left(\mathbb{R}^{N}\right), \text { para todo } 2<p<2^{*} .
$$

Demonstração. Vamos considerar o caso $N \geq 3$. Considere $q<s<2^{*}$ e $u \in H^{1}\left(\mathbb{R}^{N}\right)$. Pela desigualdade da interpolação e a imersão de Sobolev, temos que

$$
\begin{aligned}
{\left[\int_{B_{R}(y)}|u|^{s} d x\right]^{1 / s}=\|u\|_{L^{s}\left(B_{R}(y)\right)} } & \leq\|u\|_{L^{q}\left(B_{R}(y)\right)}^{1-\lambda}\|u\|_{L^{2^{*}}\left(B_{R}(y)\right)}^{\lambda} \\
& \leq C\|u\|_{L^{q}\left(B_{R}(y)\right)}^{1-\lambda}\|u\|_{H^{1}\left(B_{R}(y)\right)}^{\lambda} \\
& =C\|u\|_{L^{q}\left(B_{R}(y)\right)}^{1-\lambda}\left[\int_{B_{R}(y)}\left(|u|^{2}+|\nabla u|^{2}\right) d x\right]^{\frac{\lambda}{2}}
\end{aligned}
$$


$\log O$

$$
\int_{B_{R}(y)}|u|^{s} d x \leq C^{s}\|u\|_{L^{q}\left(B_{R}(y)\right)}^{(1-\lambda) s}\left[\int_{B_{R}(y)}\left(|u|^{2}+|\nabla u|^{2}\right) d x\right]^{\frac{\lambda}{2} s}
$$

onde $\lambda:=\left(\frac{s-q}{2^{*}-q}\right)\left(\frac{2^{*}}{s}\right)$, note que $0<\lambda<1$, já que $q<s<2^{*}$. Escolhendo $\lambda=\frac{2}{s}$ obtemos $(1-\lambda) s=s-2 \mathrm{e}$

$$
\int_{B_{R}(y)}|u|^{s} d x \leq C^{s}\|u\|_{L^{q}\left(B_{R}(y)\right)}^{s-2} \int_{B_{R}(y)}\left(|u|^{2}+|\nabla u|^{2}\right) d x .
$$

Considere agora uma família de bolas $\left\{B_{R}\left(y_{i}\right)\right\}_{i \in \mathbb{N}}$ que cobrem $\mathbb{R}^{N}$, de modo que cada ponto de $\mathbb{R}^{N}$ esteja contido em no máximo $N+1$ bolas, logo temos que

$$
\begin{aligned}
\int|u|^{s} d x & =\int_{\bigcup_{i=1}^{\infty} B_{R}\left(y_{i}\right)}|u|^{s} d x \\
& \leq \sum_{i=1}^{\infty} \int_{B_{R}\left(y_{i}\right)}|u|^{s} d x \\
& \leq \sum_{i=1}^{\infty} C^{s}\|u\|_{L^{q}\left(B_{R}\left(y_{i}\right)\right)}^{s-2} \int_{B_{R}\left(y_{i}\right)}\left(|u|^{2}+|\nabla u|^{2}\right) d x \\
& \leq C^{s} \sup _{i \in \mathbb{N}}\|u\|_{L^{q}\left(B_{R}\left(y_{i}\right)\right)}^{s-2} \sum_{i=1}^{\infty} \int\left(|u|^{2}+|\nabla u|^{2}\right) \cdot \chi_{B_{R}\left(y_{i}\right)} d x \\
& \leq C^{s} \sup _{y \in \mathbb{R}^{N}}\|u\|_{L^{q}\left(B_{R}(y)\right)}^{s-2}\left(|u|^{2}+|\nabla u|^{2}\right) \sum_{i=1}^{\infty} \chi_{B_{R}\left(y_{i}\right)} d x \\
& \leq(N+1) C^{s} \sup _{y \in \mathbb{R}^{N}}\|u\|_{L^{q}\left(B_{R}(y)\right)}^{s-2} \int\left(|u|^{2}+|\nabla u|^{2}\right) d x
\end{aligned}
$$

onde usamos o Teorema da Convergência Monótona de Lebesgue na penúltima desigualdade e

$$
\chi_{B_{R}\left(y_{i}\right)}(x)= \begin{cases}1, & \text { se } x \in B_{R}\left(y_{i}\right) \\ 0, & \text { se } x \notin B_{R}\left(y_{i}\right)\end{cases}
$$

Aplicando a desigualdade acima para $\left(u_{n}\right)$ e usando as hipóteses, chegamos em $u_{n} \rightarrow 0$, em $L^{s}\left(\mathbb{R}^{N}\right)$. Como $2<s<2^{*}$, então pelas desigualdades da interpolação e a imersão de Sobolev $H^{1}\left(\mathbb{R}^{N}\right) \hookrightarrow L^{r}\left(\mathbb{R}^{N}\right)$, para $2 \leq r \leq 2^{*}$, temos que

(a) se $2<p \leq s$, então

$$
\left\|u_{n}\right\|_{p} \leq\left\|u_{n}\right\|_{2}^{\beta}\left\|u_{n}\right\|_{s}^{1-\beta} \leq C\left\|u_{n}\right\|_{s}^{1-\beta}, \quad \text { onde } \beta=\left(\frac{s-p}{s-2}\right)\left(\frac{2}{p}\right) ;
$$

(b) se $s \leq p<2^{*}$, então

$$
\left\|u_{n}\right\|_{p} \leq\left\|u_{n}\right\|_{s}^{\mu}\left\|u_{n}\right\|_{2^{*}}^{1-\mu} \leq C\left\|u_{n}\right\|_{s}^{1-\mu}, \quad \text { onde } \quad \mu=\left(\frac{s-p}{s-2^{*}}\right)\left(\frac{2^{*}}{p}\right) .
$$

Logo, desde que $u_{n} \rightarrow 0$, em $L^{s}\left(\mathbb{R}^{N}\right)$ obtemos que $\left\|u_{n}\right\|_{p} \rightarrow 0$, para $2<p<2^{*}$, e temos o resultado. 


\subsection{Análise Funcional}

As demonstrações que apresentaremos a seguir podem ser vistas com maiores detalhes em Brezis [5] e Kreyszig [13].

Definição 1.2. Um espaço $H$ com produto interno é dito espaço de Hilbert se $H$ é completo com a norma induzida pelo produto interno.

Teorema 1.4. (Teorema de Representação de Riesz) Seja H um espaço de Hilbert com produto interno $\langle\cdot, \cdot\rangle_{H}$. Dado $g \in H^{*}$, existe um único $\bar{u} \in H$ tal que

$$
\langle\bar{u}, x\rangle_{H}=g(x), \text { para todo } x \in H
$$

Demonstração. Se $g=0$, então (1.5) é verdadeiro para $\bar{u}=0$. Seja então $g \neq 0$ e considere o núcleo de $g$, que é o espaço vetorial fechado denotado por $N(g)$. Como $g \neq 0$ então $N(g) \neq H$, e segue que o complemento ortogonal de $N(g)$ não é nulo, ou seja, $N^{\perp}(g) \neq 0$. Tome então $0 \neq u_{0} \in N^{\perp}(g)$ e defina

$$
v=g(x) u_{0}-g\left(u_{0}\right) x
$$

onde $x \in H$ é arbitrário. Aplicando $g$, obtemos

$$
g(v)=g(x) g\left(u_{0}\right)-g\left(u_{0}\right) g(x)=0 .
$$

Isto nos mostra que $v \in N(g)$. Como $u_{0} \perp N(g)$, temos

$$
\begin{aligned}
0 & =\left\langle v, u_{0}\right\rangle_{H} \\
& =\left\langle g(x) u_{0}-g\left(u_{0}\right) x, u_{0}\right\rangle_{H} \\
& =g(x)\left\langle u_{0}, u_{0}\right\rangle_{H}-g\left(u_{0}\right)\left\langle x, u_{0}\right\rangle_{H} .
\end{aligned}
$$

Como $\left\langle u_{0}, u_{0}\right\rangle_{H}=\left\|u_{0}\right\|_{H}^{2} \neq 0$, obtemos

$$
g(x)=\frac{g\left(u_{0}\right)}{\left\langle u_{0}, u_{0}\right\rangle_{H}}\left\langle x, u_{0}\right\rangle_{H}=\left\langle x, \frac{g\left(u_{0}\right)}{\left\langle u_{0}, u_{0}\right\rangle_{H}} u_{0}\right\rangle_{H} .
$$

Se escrevermos

$$
\bar{u}=\frac{g\left(u_{0}\right)}{\left\langle u_{0}, u_{0}\right\rangle_{H}} u_{0}
$$

obtemos

$$
g(x)=\langle x, \bar{u}\rangle_{H}
$$

e como $x \in H$ é arbitrário, fica provado (1.5).

Para provar a unicidade, suponha que, para todo $x \in H$, tenhamos

$$
g(x)=\left\langle x, u_{1}\right\rangle_{H}=\left\langle x, u_{2}\right\rangle_{H},
$$

então $\left\langle x, u_{1}-u_{2}\right\rangle_{H}=0$ para todo $x$. Em particular para $x=u_{1}-u_{2}$, temos

$$
\left\langle u_{1}-u_{2}, u_{1}-u_{2}\right\rangle_{H}=\left\|u_{1}-u_{2}\right\|_{H}^{2}=0
$$


portanto $u_{1}-u_{2}=0$, de modo que vale a unicidade.

Definição 1.3. Um espaço vetorial normado $\left(X,\|\cdot\|_{X}\right)$ é dito espaço de Banach se $X$ é completo com a norma $\|u\|_{X}$.

Definição 1.4. Seja $X$ um espaço vetorial normado e $\left(x_{n}\right) \subset X$ uma sequência. Dizemos que $\left(x_{n}\right)$ converge fracamente em $X$, se existe $x \in X$ tal que, para toda $f \in X^{*}$, tenhamos $\left\langle f, x_{n}\right\rangle \rightarrow\langle f, x\rangle$. Denotamos este fato por $x_{n} \rightarrow x$.

Teorema 1.5. Seja $\left(x_{n}\right)$ uma sequência em um espaço vetorial normado $X$.

(i) se $x_{n} \rightarrow x$, então $x_{n} \rightarrow x$ em $X$;

(ii) se $x_{n} \rightarrow x$ em $X$, então $\left(x_{n}\right)$ é limitada e $\|x\|_{X} \leq \liminf \left\|x_{n}\right\|_{X}$;

(iii) se $x_{n} \rightarrow x$ em $X$ e $f_{n} \rightarrow f$ em $X^{*}$, entã $\left\langle\left\langle f_{n}, x_{n}\right\rangle \stackrel{n \rightarrow \infty}{\rightarrow}\langle f, x\rangle\right.$.

Demonstração. Proposição 3.5, página 58 de Brezis [5].

Teorema 1.6. Seja $X$ em espaço de Banach reflexivo $e\left(x_{n}\right) \subset X$ uma sequência limitada. Então existe uma subsequência $\left(x_{n_{k}}\right) \subset\left(x_{n}\right)$ tal que $x_{n_{k}} \rightarrow x$ em $X$.

Demonstração. Pelo teorema de Kakutani (Teorema 3.17, página 67 de Brezis [5]), temos que a bola unitária de $X$ é fracamente compacta. Tome $\left(x_{n}\right)$ limitada, logo $\left(x_{n}\right) \subset \overline{B_{R}}$, para algum $R>0$, e por $\overline{B_{R}}$ ser fracamente compacta, temos que existe uma subsequência $\left(x_{j}\right) \subset\left(x_{n}\right)$ tal que $x_{j} \rightarrow x$, em $X$.

Teorema 1.7. Sejam $H$ um espaço de Hilbert separável e $T: H \rightarrow H$ um operador compacto e autoadjunto. Então $H$ admite uma base hilbertiana formada por autofunções de $T$, ou seja, admite uma base $\left(u_{j}\right)$ tal que $T u_{j}=\mu_{j} u_{j},\left\langle u_{i}, u_{j}\right\rangle_{H}=0$ para $i \neq j e\left\langle u_{j}, u_{j}\right\rangle_{H}=1$. Além disso, a dimensão de qualquer autoespaço é finita.

Demonstração. Teorema 6.11, página 167 de Brezis [5].

Seja agora um espaço normado real $E$ e $T: D(T) \subset E \rightarrow E$ linear. Para cada $\lambda \in \mathbb{R}$ defina

$$
\begin{aligned}
T_{\lambda}: D(T) & \rightarrow E \\
u & \mapsto T u-\lambda u,
\end{aligned}
$$

ou seja, $T_{\lambda}=T-\lambda I$.

Definição 1.5. O operador

$$
\begin{aligned}
R_{\lambda}: T_{\lambda}(D(T)) & \rightarrow D(T) \\
T_{\lambda} u & \mapsto u,
\end{aligned}
$$

quando existir, é chamado de operador resolvente de T. Em outras palavras $R_{\lambda}=T_{\lambda}^{-1}$.

Definição 1.6. Dizemos que $\lambda \in \mathbb{R}$ é um valor regular de $T$ se:

(i) $R_{\lambda}$ existir;

(ii) $R_{\lambda}$ for continuo;

(iii) $\overline{D\left(R_{\lambda}\right)}=E$. 
Definição 1.7. Com relação a um operador $T$, temos:

a) $O$ conjunto dos números reais que são valores regulares de $T$, denotado por $\rho(T)$, é chamado de resolvente de $T$.

b) $O$ complementar em $\mathbb{R}$ do resolvente de $T$, denotado por $\sigma(T)$, é chamado de espectro de $T$.

Teorema 1.8. Sejam $E$ um espaço normado com dimensão infinita e $T: E \rightarrow E$ um operador linear compacto. Então:

(1) $0 \in \sigma(T)$;

(2) $\sigma(T) \backslash\{0\}=A(T) \backslash\{0\}$, onde $A(T)$ é o conjuntos dos autovalores de $T$;

(3) Ocorre apenas uma das seguintes alternativas:

(i) $\sigma(T)=\{0\}$;

(ii) $\sigma(T) \backslash\{0\}$ é finito e, portanto, discreto;

(iii) $\sigma(T) \backslash\{0\}=\mu_{n} \rightarrow 0$.

Demonstração. Teorema 6.8, página 164 de Brezis [5].

\subsection{Teoria da Medida}

Mostraremos aqui resultados de Medida e Integração que serão usados tanto explícita quanto implicitamente neste trabalho. Os próximos resultados podem ser consultados em Bartle [3] e Brezis [5].

Seja $(A, \mathbf{A}, \mu)$ um espaço de medida onde $A$ é um conjunto, $\mathbf{A}$ é uma $\sigma$-álgebra e $\mu$ é uma medida. Denotaremos por $\mathbf{M}^{+}(A, \mathbf{A})$ as funções $\mathbf{A}$-mensuráveis não negativas de $A$ para $\mathbb{R}^{e}=\mathbb{R} \cup\{\infty\}$.

Teorema 1.9. (Teorema da Convergência Monótona de Lebesgue) Sejam $(A, \boldsymbol{A}, \mu)$ um espaço de medida $e\left(f_{n}\right)$ uma sequência de funçôes mensuráveis em $A$, e suponha que:

(i) $0 \leq f_{1} \leq f_{2} \leq \ldots$, em quase todo ponto de $A$;

(ii) $f_{n} \rightarrow f$, em quase todo ponto de A.

Então $f$ é mensurável, $e$

$$
\int_{A} f_{n} d \mu \rightarrow \int_{A} f d \mu
$$

quando $n \rightarrow \infty$.

Demonstração. Sabemos que o limite de um sequência de funções mensuráveis é mensurável, $\operatorname{logo} f$ é mensurável. Como $f_{n} \leq f_{n+1} \leq f, \forall n \in \mathbb{N}$, segue que

$$
\int_{A} f_{n} d \mu \leq \int_{A} f_{n+1} d \mu \leq \int_{A} f d \mu .
$$

Portanto

$$
\lim _{n \rightarrow \infty} \int_{A} f_{n} d \mu \leq \int_{A} f d \mu .
$$

Para obtermos a desigualdade oposta, seja $\alpha \in(0,1)$ e seja $\varphi$ uma função simples com $0 \leq \varphi \leq f$. Defina

$$
A_{n}=\left\{x \in A \mid f_{n}(x) \geq \alpha \varphi(x)\right\},
$$


$\log 0 A_{n} \in \mathbf{A}, A_{n} \subset A_{n+1}$, e $A=\bigcup_{n=1}^{\infty} A_{n}$. Teremos então

$$
\int_{A_{n}} \alpha \varphi d \mu \leq \int_{A_{n}} f_{n} d \mu \leq \int_{A} f_{n} d \mu .
$$

Como a sequência $\left(A_{n}\right)$ é monótona crescente e $A=\bigcup_{n=1}^{\infty} A_{n}$, segue que

$$
\int_{A} \varphi d \mu=\lim _{n \rightarrow \infty} \int_{A_{n}} \varphi d \mu
$$

Tomando o limite em (1.7), obtemos

$$
\alpha \int_{A} \varphi d \mu \leq \lim _{n \rightarrow \infty} \int_{A} f_{n} d \mu
$$

e como esta desigualdade vale para todo $\alpha \in(0,1)$, concluímos que

$$
\int_{A} \varphi d \mu \leq \lim _{n \rightarrow \infty} \int_{A} f_{n} d \mu .
$$

Como $\varphi$ é uma função simples arbitrária satisfazendo $0 \leq \varphi \leq f$, chegamos em

$$
\int_{A} f d \mu=\sup _{\varphi} \int_{A} \varphi d \mu \leq \lim _{n \rightarrow \infty} \int_{A} f_{n} d \mu .
$$

Combinando (1.6) e (1.8), obtemos o resultado.

Lema 1.3. (Lema de Fatou) $S e\left(f_{n}\right) \subset \boldsymbol{M}^{+}(A, \boldsymbol{A})$, então

$$
\int_{A}\left(\liminf _{n \rightarrow \infty} f_{n}\right) d \mu \leq \liminf _{n \rightarrow \infty} \int_{A} f_{n} d \mu .
$$

Demonstração. Seja $g_{m}=\inf \left\{f_{m}, f_{m+1, \ldots}\right\}$ de modo que $g_{m} \leq f_{n}$ sempre que $m \leq n$. Assim temos

$$
\int_{A} g_{m} d \mu \leq \int_{A} f_{n} d \mu
$$

$\log 0$

$$
\int_{A} g_{m} d \mu \leq \liminf _{n \rightarrow \infty} \int_{A} f_{n} d \mu .
$$

Como a sequência $\left(g_{m}\right)$ é não decrescente e converge para $\liminf _{n \rightarrow \infty} f_{n}$, o Teorema 1.9 da Convergência Monótona implica que

$$
\int_{A} \liminf _{n \rightarrow \infty} f_{n} d \mu=\lim _{n \rightarrow \infty} \int_{A} g_{m} d \mu \leq \liminf _{n \rightarrow \infty} \int_{A} f_{n} d \mu
$$

Teorema 1.10. (Teorema da Convergência Dominada) Sejam $(A, \boldsymbol{A}, \mu)$ um espaço de medida e $\left(f_{n}\right)$ 
uma sequência de funções mensuráveis em A, tal que

$$
f_{n} \rightarrow f, \text { em quase todo ponto de A. }
$$

Se existe uma função integrável g tal que

$$
\left|f_{n}\right| \leq g \text {, em quase todo ponto de } A,
$$

então $f$ é integrável $e$

$$
\int_{A} f d \mu=\lim _{n \rightarrow \infty} \int_{A} f_{n} d \mu .
$$

Demonstração. Temos que $f$ é integrável e como $g+f_{n} \geq 0$, podemos aplicar o Lema 1.3 de Fatou para obter

$$
\begin{aligned}
\int_{A} g d \mu+\int_{A} f d \mu & =\int_{A}(g+f) d \mu \\
& \leq \liminf _{n \rightarrow \infty} \int_{A}\left(g+f_{n}\right) d \mu \\
& =\liminf _{n \rightarrow \infty}\left(\int_{A} g d \mu+\int_{A} f_{n} d \mu\right) \\
& =\int_{A} g d \mu+\liminf _{n \rightarrow \infty} \int_{A} f_{n} d \mu
\end{aligned}
$$

Segue que

$$
\int_{A} f d \mu \leq \liminf _{n \rightarrow \infty} \int_{A} f_{n} d \mu
$$

Como $g-f_{n} \geq 0$, mais uma aplicação do Lema 1.3 de Fatou nos dá

$$
\begin{aligned}
\int_{A} g d \mu-\int_{A} f d \mu & =\int_{A}(g-f) d \mu \\
& \leq \liminf _{n \rightarrow \infty} \int_{A}\left(g-f_{n}\right) d \mu \\
& =\int_{A} g d \mu-\limsup _{n \rightarrow \infty} \int_{A} f_{n} d \mu
\end{aligned}
$$

ou seja,

$$
\limsup _{n \rightarrow \infty} \int_{A} f_{n} d \mu \leq \int_{A} f d \mu
$$

Assim, concluímos que

$$
\int_{A} f d \mu=\lim _{n \rightarrow \infty} \int_{A} f_{n} d \mu .
$$

Definição 1.8. Sejam $(A, \boldsymbol{A}, \mu)$ um espaço de medida, $f: A \rightarrow \mathbb{R}$ uma função mensurável $e 1 \leq p<\infty$. Definimos

$$
\|f\|_{p}=\left[\int_{A}|f|^{p} d \mu\right]^{1 / p}
$$


e $L^{p}(A)$ a coleção de todas as funções mensuráveis em A tais que

$$
\|f\|_{p}<\infty
$$

Definimos também $L^{\infty}(A)$ como o espaço vetorial de todas as funções mensuráveis $f$ tais que $|f(x)| \leq M$, em quase todo ponto de $A$, para algum $M>0$. Definimos a norma $\|f\|_{\infty}$ em $L^{\infty}(A)$ por

$$
\|f\|_{\infty}=\inf \{M>0|| f \mid<M \text {, em quase todo ponto de } A\} .
$$

Teorema 1.11. (Desigualdade de Hölder) Consideremos $(A, \boldsymbol{A}, \mu)$ um espaço de medida e $1 \leq p, q \leq \infty$ com $\frac{1}{p}+\frac{1}{q}=1$. Se $f \in L^{p}(A), g \in L^{q}(A)$, então $f g \in L^{1}(A) e$

$$
\|f g\|_{1} \leq\|f\|_{p}\|g\|_{q}
$$

Demonstração. Suponha $p=1$ e $q=\infty, \operatorname{logo}$

$$
\begin{aligned}
\left|\int_{A} f g d \mu\right| & \leq \int_{A}|f g| d \mu \\
& \leq\|g\|_{\infty} \int_{A}|f| d \mu \\
& =\|g\|_{\infty}\|f\|_{1} \leq \infty
\end{aligned}
$$

e temos o resultado no caso $p=1$.

Para o caso $p>1$, seja $\alpha \in(0,1)$ e $\varphi(t):=\alpha t-t^{\alpha}$ para $t \geq 0$. Logo, $\varphi^{\prime}(t)=\alpha-\alpha t^{\alpha-1}$ e assim $\varphi^{\prime}(t)<0$ para $0<t<1$ e $\varphi^{\prime}(t)>0$ para $t>1$. Logo, $t=1$ é ponto de mínimo de $\varphi$, ou seja, $\varphi(t) \geq \varphi(1)$ e $\varphi(t)=\varphi(1)$ se, e somente se, $t=1$.

Temos então que $\varphi(t) \geq \varphi(1)$ implica em

$$
t^{\alpha} \leq \alpha t+(1-\alpha), \quad t \geq 0
$$

Sejam $a, b$ não negativos e $t=\frac{a}{b}$, logo teremos

$$
a^{\alpha} b^{-\alpha} \leq \alpha a b^{-1}+(1-\alpha)
$$

e multiplicando por $b$, temos

$$
a^{\alpha} b^{1-\alpha} \leq \alpha a+(1-\alpha) b
$$

onde a igualdade vale se, e somente se, $a=b$.

Sejam agora $p$ e $q$ satisfazendo $1<p<\infty$ e $\frac{1}{p}+\frac{1}{q}=1$ e tome $\alpha=\frac{1}{p}$. Segue que se $A$ e $B$ são números reais não negativos, então

$$
A B \leq \frac{A^{p}}{p}+\frac{B^{q}}{q},
$$

e a igualdade ocorre se, e somente se, $A^{p}=B^{q}$.

Suponha que $f \in L^{p}, g \in L^{q}$ e $\|f\|_{p},\|g\|_{q} \neq 0$, então o produto $f g$ é mensurável e tomando $A=\frac{|f(x)|}{\|f\|_{p}}$ 
e $B=\frac{|g(x)|}{\|g\|_{q}}$, obtemos

$$
\frac{|f(x) g(x)|}{\|f\|_{p}\|g\|_{q}} \leq \frac{|f(x)|^{p}}{p\|f\|_{p}^{p}}+\frac{|g(x)|^{q}}{q\|g\|_{q}^{q}} .
$$

Como os dois termos à direita são integráveis, segue que $f g$ é integrável. Além disso, integrando, obtemos

$$
\frac{\|f g\|_{1}}{\|f\|_{p}\|g\|_{q}} \leq \frac{1}{p}+\frac{1}{q}=1
$$

o que prova nosso resultado.

Teorema 1.12. (Desigualdade de Interpolação) Sejam $1 \leq s \leq r \leq t \leq \infty$ e $\theta \in(0,1)$ tal que

$$
\frac{1}{r}=\frac{\theta}{s}+\frac{1-\theta}{t}
$$

Suponhamos também que $u \in L^{s}(\Omega) \cap L^{t}(\Omega)$, onde $\Omega$ é um domínio limitado. Então, $u \in L^{r}(\Omega) e$

$$
\|u\|_{r} \leq\|u\|_{s}^{\theta}\|u\|_{t}^{1-\theta} .
$$

Demonstração. Usando a desigualdade de Hölder com os expoentes conjugados $\frac{\theta r}{s}+\frac{(1-\theta) r}{t}=1$, obtemos

$$
\begin{aligned}
\|u\|_{r}^{r} & \leq \int_{\Omega}|u|^{r} d x \\
& =\int_{\Omega}|u|^{\theta r}|u|^{(1-\theta) r} d x \\
& \leq\left(\int_{\Omega}|u|^{\theta r \frac{s}{\theta r}} d x\right)^{\frac{\theta r}{s}}\left(\int_{\Omega}|u|^{(1-\theta) r \frac{t}{(1-\theta) r}} d x\right)^{\frac{(1-\theta) r}{t}} \\
& =\left(\int_{\Omega}|u|^{s} d x\right)^{\frac{\theta r}{s}}\left(\int_{\Omega}|u|^{t} d x\right)^{\frac{(1-\theta) r}{t}} \\
& =\|u\|_{s}^{\theta r}\|u\|_{t}^{(1-\theta) r},
\end{aligned}
$$

$\log 0$

$$
\|u\|_{r} \leq\|u\|_{s}^{\theta}\|u\|_{t}^{(1-\theta)},
$$

e como $u \in L^{s}(\Omega) \cap L^{t}(\Omega)$, temos o resultado.

Teorema 1.13. Considere uma sequência $\left(f_{n}\right) \subset L^{p}(\Omega)$ e $f \in L^{p}(\Omega)$, de modo que $\left\|f_{n}-f\right\|_{p} \rightarrow 0$, quando $n \rightarrow \infty$. Então existe uma subsequência $\left(f_{n_{k}}\right)$ tal que:

(i) $f_{n_{k}}(x) \rightarrow f(x)$, em quase todo ponto de $\Omega$;

(ii) $\left|f_{n_{k}}(x)\right| \leq g(x)$, em quase todo ponto de $\Omega$, com $g \in L^{p}(\Omega)$.

Demonstração. Teorema 4.9, página 94 de Brezis [5]. 


\subsection{Espaços de Sobolev}

Nesta seção, vamos abordar conceitos e resultados sobre os Espaços de Sobolev que serão utilizados por todo este trabalho. Os detalhes desta seção podem ser vistos em Brezis [5] e Evans [8].

Seja $\Omega$ um aberto de $\mathbb{R}^{N}, p \in \mathbb{R}$, com $1 \leq p \leq \infty$ e $k \in \mathbb{N}$.

Definição 1.9. O espaço de Sobolev $W^{k, p}(\Omega)$ é o conjunto de todas as funções $u \in L^{p}(\Omega)$ tais que, para todo multi-índice $\alpha$, com $|\alpha| \leq k$, tem-se $D^{\alpha} u \in L^{p}(\Omega)$, sendo $D^{\alpha}$ a derivada no sentido fraco. De forma sucinta,

$$
W^{k, p}(\Omega)=\left\{u \in L^{p}(\Omega)\left|D^{\alpha} u \in L^{p}(\Omega), \forall \alpha \operatorname{com}\right| \alpha \mid \leq k\right\} .
$$

Definição 1.10. Para $u \in W^{k, p}$ definimos a norma de $u$ por

$$
\|u\|_{W^{k, p}(\Omega)}=\left(\sum_{|\alpha| \leq k}\left\|D^{\alpha} u\right\|_{p}^{p}\right)^{1 / p}, \quad 1 \leq p<\infty
$$

$$
\|u\|_{W^{k, \infty}(\Omega)}=\sum_{|\alpha| \leq k}\left\|D^{\alpha} u\right\|_{\infty} .
$$

Observação 1.1. Se $p=2$, escrevemos $H^{k}(\Omega)=W^{k, 2}(\Omega)$.

Teorema 1.14. O espaço de Sobolev $W^{1, p}(\Omega)$ é um espaço de Banach para $1 \leq p \leq \infty$, reflexivo para $1<p<\infty$ e separável para $1 \leq p<\infty$.

Demonstração. Proposição 8.1, página 203 de Brezis [5].

Veremos agora as imerões dos espaços de Sobolev $W^{1, p}(\Omega)$. Lembramos agora que o expoente crítico de Sobolev é dado por

$$
p^{*}=\frac{N p}{N-p}, \quad \text { onde } 1 \leq p<N .
$$

Teorema 1.15. Seja $\Omega \subset \mathbb{R}^{N}$ um aberto limitado de classe $C^{1}$, então temos as seguintes imersões continuas:

$$
\begin{array}{lll}
W^{1, p}(\Omega) & \hookrightarrow L^{q}(\Omega), \quad 1 \leq q \leq p^{*}, & \text { se } 1 \leq p<N ; \\
W^{1, p}(\Omega) & \hookrightarrow L^{q}(\Omega), \quad q \geq 1, & \text { se } p=N ; \\
W^{1, p}(\Omega) & \hookrightarrow C^{0,1-\frac{N}{p}}(\bar{\Omega}), & \text { se } p>N .
\end{array}
$$

Demonstração. Teorema 5.4, página 97 de Adams [1].

\subsection{Caracterização de $\lambda_{1}$}

No que segue estamos nos baseando em Giovany [9]. Inicialmente vamos considerar o problema

$$
(P) \quad \begin{cases}-\Delta u=f(x), & \text { em } \Omega \\ u=0, & \text { sobre } \partial \Omega,\end{cases}
$$

onde $\Omega \subset \mathbb{R}^{N}$ é um domínio limitado e $f \in L^{2}(\Omega)$. 
Sabemos que

$$
\langle u, v\rangle_{H}=\int_{\Omega} \nabla u \nabla v d x
$$

é o produto interno usual de $H_{0}^{1}(\Omega)$, que é espaço de Hilbert com a norma induzida

$$
\|u\|_{H}=\left(\int_{\Omega}|\nabla u|^{2} d x\right)^{1 / 2} .
$$

Dada $f \in L^{2}(\Omega)$, considere $g: H_{0}^{1}(\Omega) \rightarrow \mathbb{R}$ definido por

$$
g(v)=\int_{\Omega} f v d x
$$

Logo, pela desigualdade de Hölder, $g$ está bem definido e além disso, $g$ é linear.

Tomando $v \in H_{0}^{1}(\Omega)$ e usando as imersões de Sobolev e novamente a desigualdade de Hölder, temos

$$
|g(v)| \leq\|f\|_{L^{2}}\|v\|_{L^{2}} \leq C\|f\|_{L^{2}}\|v\|_{H}
$$

o que mostra que $g$ é contínua.

Pelo Teorema 1.4 da Representação de Riesz, segue que existe único $\bar{u} \in H_{0}^{1}(\Omega)$ tal que

$$
\langle\bar{u}, v\rangle_{H}=g(v), \text { para todo } v \in H_{0}^{1}(\Omega),
$$

ou seja,

$$
\int_{\Omega} \nabla \bar{u} \nabla v d x=\int_{\Omega} f v d x, \text { para todo } v \in H_{0}^{1}(\Omega) .
$$

Concluímos então que $\bar{u} \in H_{0}^{1}(\Omega)$ é a única solução fraca do problema $(P)$.

Assim fica bem definido o operador

$$
\begin{aligned}
S: L^{2}(\Omega) & \rightarrow H_{0}^{1}(\Omega) \\
f & \mapsto u,
\end{aligned}
$$

onde $u$ é a única solução do problema $(P)$.

Vamos agora conhecer as propriedades do operador $S$ :

Lema 1.4. O operador $S$ é linear e contínuo.

Demonstração. Dadas $f_{1}, f_{2} \in L^{2}(\Omega)$ e $\alpha \in \mathbb{R}$, obtemos únicas $u_{1}, u_{2} \in H_{0}^{1}(\Omega)$ tais que $S\left(f_{1}\right)=u_{1}$ e $S\left(f_{2}\right)=u_{2}$, ou seja,

$$
\int_{\Omega} \nabla u_{1} \nabla v d x=\int_{\Omega} f_{1} v d x, \quad \forall v \in H_{0}^{1}(\Omega)
$$

e

$$
\int_{\Omega} \nabla u_{2} \nabla v d x=\int_{\Omega} f_{2} v d x, \forall v \in H_{0}^{1}(\Omega)
$$

Multiplicando a segunda equação por $\alpha$ e somando com a primeira equação, teremos

$$
\int_{\Omega} \nabla u_{1} \nabla v d x+\alpha \int_{\Omega} \nabla u_{2} \nabla v d x=\int_{\Omega} f_{1} v d x+\alpha \int_{\Omega} f_{2} v d x
$$


ou seja,

$$
\int_{\Omega} \nabla\left(u_{1}+\alpha u_{2}\right) \nabla v d x=\int_{\Omega}\left(f_{1}+\alpha f_{2}\right) v d x, \quad \forall v \in H_{0}^{1}(\Omega) .
$$

O que mostra que

$$
S\left(f_{1}+\alpha f_{2}\right)=S\left(f_{1}\right)+\alpha S\left(f_{2}\right) .
$$

Vamos mostrar agora que $S$ é contínuo. Da desigualdade de Hölder e das imersões de Sobolev, temos

$$
\begin{aligned}
\|S(f)\|_{H}^{2} & =\|u\|_{H}^{2} \\
& =\int_{\Omega} f u d x \\
& \leq\|u\|_{L^{2}}\|f\|_{L^{2}} \\
& \leq C\|u\|_{H}\|f\|_{L^{2}} \\
& =C\|S(f)\|_{H}\|f\|_{L^{2}},
\end{aligned}
$$

e assim

$$
\|S(f)\|_{H} \leq C\|f\|_{L^{2}}
$$

mostrando que $S$ é contínuo.

Observação 1.2. Note que:

$$
S: L^{2}(\Omega) \rightarrow H_{0}^{1}(\Omega) \hookrightarrow L^{2}(\Omega)
$$

onde a imersão acima é compacta. Como $S$ é continuo teremos

$$
S: L^{2}(\Omega) \rightarrow L^{2}(\Omega)
$$

um operador compacto. Daqui para frente o operador $S$ será o operador compacto acima.

Lema 1.5. O operador $S: L^{2}(\Omega) \rightarrow L^{2}(\Omega)$ é um operador positivo, ou seja,

$$
\langle S(f), f\rangle_{L^{2}} \geq 0
$$

Demonstração. Seja $S(f)=u$, logo

$$
\begin{aligned}
\langle S(f), f\rangle_{L^{2}} & =\langle u, f\rangle_{L^{2}} \\
& =\int_{\Omega} u f d x \\
& =\int_{\Omega} \nabla u \nabla u d x \\
& =\|u\|_{H}^{2} \geq 0 .
\end{aligned}
$$

Lema 1.6. O operador $S$ é autoadjunto, ou seja,

$$
\langle S(f), g\rangle_{L^{2}}=\langle f, S(g)\rangle_{L^{2}}
$$


Demonstração. Sejam $u_{1}=S\left(f_{1}\right)$ e $u_{2}=S\left(f_{2}\right)$, então

$$
\int_{\Omega} \nabla u_{1} \nabla v d x=\int_{\Omega} f_{1} v d x, \text { para todo } v \in H_{0}^{1}(\Omega)
$$

e

$$
\int_{\Omega} \nabla u_{2} \nabla w d x=\int_{\Omega} f_{2} w d x, \text { para todo } w \in H_{0}^{1}(\Omega) .
$$

Considerando $v=u_{2}$ em (1.9) e $w=u_{1}$ em (1.10), obtemos

$$
\int_{\Omega} f_{2} u_{1} d x=\int_{\Omega} \nabla u_{1} \nabla u_{2} d x=\int_{\Omega} f_{1} u_{2} d x
$$

o que nos mostra que

$$
\left\langle S\left(f_{1}\right), f_{2}\right\rangle_{L^{2}}=\left\langle S\left(f_{2}\right), f_{1}\right\rangle_{L^{2}}, \forall f_{1}, f_{2} \in L^{2}(\Omega)
$$

Lema 1.7. O operador $S$ é injetivo.

Demonstração. Temos que

$$
0=S(f)=\int_{\Omega} f v d x, \forall v \in H_{0}^{1}(\Omega),
$$

e como $L^{2}(\Omega) \subset L_{\text {loc }}^{1}(\Omega)$ e $C_{0}^{\infty}(\Omega) \subset H_{0}^{1}(\Omega)$, temos que $f \in L_{l o c}^{1}(\Omega)$ e

$$
\int_{\Omega} f v d x=0, \forall v \in C_{0}^{\infty}(\Omega)
$$

Assim, concluímos que $f=0$.

Observação 1.3. Segue do lema acima que o operador $S$ não possui autovalor nulo.

Lema 1.8. O operador $S: L^{2}(\Omega) \rightarrow L^{2}(\Omega)$ admite uma sequência $\mu_{n}$ de autovalores com $\lim _{n \rightarrow \infty} \mu_{n}=0 e$ $L^{2}(\Omega)$ possui uma base hilbertiana formada por autofunções de $S$.

Demonstração. Sabemos que $L^{2}(\Omega)$ é um espaço de Hilbert separável, $S: L^{2}(\Omega) \rightarrow L^{2}(\Omega)$ é um operador autoadjunto e compacto. Do Teorema $1.7, L^{2}(\Omega)$ possui uma base hilbertiana formada por autofunções de $S$.

Vamos mostrar agora que os itens $(i)$ e $(i i)$ do Teorema 1.8 não podem ocorrer. De fato, se $\sigma(S)=\{0\}$, desde que $A(T) \subset \sigma(S)$, teríamos que zero é um autovalor de $S$, o que é um absurdo. Se $\sigma(S) \backslash\{0\}$ fosse finito, do Teorema 1.7, teríamos que $L^{2}(\Omega)$ é finito, o que é um absurdo.

Temos então que

$$
\sigma(S) \backslash\{0\}=A(S) \backslash\{0\}=\mu_{n} \rightarrow 0
$$

é uma sequência que converge para zero quando $n \rightarrow \infty$.

Observação 1.4. Podemos reordenar $\left(\mu_{n}\right)$ de modo que

$$
\mu_{1} \geq \mu_{2} \geq \ldots \geq \mu_{n} \rightarrow 0
$$


Além disso, os autovalores de $S$ são positivos. De fato, seja $\mu$ autovalor de $S$, logo existe $0 \neq f \in L^{2}(\Omega)$ tal que $S(f)=\mu f$. Assim

$$
\langle S(f), f\rangle_{L^{2}}=\langle\mu f, f\rangle_{L^{2}}=\mu\|f\|_{L^{2}}^{2} \geq 0 .
$$

Portanto, $\mu \geq 0$ e como $\mu \neq 0$, então devemos ter $\mu>0$.

Definição 1.11. Dizemos que $\lambda$ é um autovalor do laplaciano se

$$
\int_{\Omega} \nabla \phi \nabla v d x=\lambda \int_{\Omega} \phi v d x
$$

para todo $v \in H_{0}^{1}(\Omega)$.

Lema 1.9. Temos que $\mu$ é autovalor de $S$ se, e somente se, $\lambda=\frac{1}{\mu}$ é autovalor de $\left(-\Delta, H_{0}^{1}(\Omega)\right)$.

Demonstração. Se $\mu$ é autovalor de $S$, então existe $0 \neq f \in L^{2}(\Omega)$ tal que

$$
S(f)=\mu f,
$$

$\log 0$

$$
\begin{aligned}
\int_{\Omega} \nabla(\mu f) \nabla v d x & =\int_{\Omega} f v d x, \quad \text { ou seja, } \\
\int_{\Omega} \nabla f \nabla v d x & =\lambda \int_{\Omega} f v d x
\end{aligned}
$$

onde $\lambda=\frac{1}{\mu}$.

Observação 1.5. Concluimos então que o operador $\left(-\Delta, H_{0}^{1}(\Omega)\right)$ possui uma sequência de autovalores $\left(\lambda_{n}\right)$ tal que

$$
\lambda_{1} \leq \lambda_{2} \leq \ldots \leq \lambda_{n} \rightarrow \infty .
$$

Teorema 1.16. O inverso do primeiro autovalor do operador $\left(-\Delta, H_{0}^{1}(\Omega)\right)$ é a menor constante que verifica a desigualdade de Poincaré, ou seja

$$
\int_{\Omega}|u|^{2} d x \leq \frac{1}{\lambda_{1}} \int_{\Omega}|\nabla u|^{2} d x
$$

Além disso

$$
\lambda_{1}=\min _{0 \neq u \in H_{0}^{1}(\Omega)} \frac{\|u\|_{H}^{2}}{\|u\|_{2}^{2}}=\min _{0 \neq u \in H_{0}^{1}(\Omega)} \frac{\int_{\Omega}|\nabla u|^{2} d x}{\int_{\Omega}|u|^{2} d x}
$$

é a caracterização variacional de $\lambda_{1}$.

Demonstração. Seção 4.5, página 50 de Figueiredo [9].

Teorema 1.17. O primeiro autovalor de $\left(-\Delta, H_{0}^{1}(\Omega)\right)$ é o único que tem a autofunção correspondente que não troca de sinal em $\Omega$.

Demonstração. Lema 4.8, página 53 de Figueiredo [9]. 


\section{Capítulo}

2

\section{Estrutura Variacional - A Condição de Cerami}

Começamos apresentando nosso principal problema, bem como as condições sobre a função $f$.

\subsection{Condições para $f$}

Neste capítulo, vamos considerar a questão de achar soluções positivas da equação

$$
\left(P_{\lambda}\right) \quad-\Delta u+\lambda u=f(x, u) u, \quad x \in \mathbb{R}^{N},
$$

onde $\lambda>0$ é um parâmetro e a função $f$ satisfaz as seguintes condições:

$\left(f_{1}\right) f \in C\left(\mathbb{R}^{N} \times \mathbb{R}^{+}, \mathbb{R}^{+}\right)$,

$$
\lim _{s \rightarrow 0} f(x, s)=0, \quad \text { uniformemente em } \quad x
$$

$\left(f_{2}\right)$ Para todo $x \in \mathbb{R}^{N}, f(x, s)$ é uma função não decrescente de $s$ em $[0, \infty)$ e existe uma função $g \in C\left(\mathbb{R}^{N}, \mathbb{R}^{+}\right)$tal que

$$
\lim _{s \rightarrow \infty} f(x, s)=g(x), \quad \text { uniformemente em } x ;
$$

$\left(f_{3}\right)$ Existe uma função $h \in C\left(\mathbb{R}^{+}, \mathbb{R}^{+}\right)$tal que

$$
\lim _{|x| \rightarrow \infty} f(x, s)=h(s), \quad \text { uniformemente em } s
$$

$\left(f_{4}\right) \lim _{|x| \rightarrow \infty, s \rightarrow \infty} f(x, s)=\lim _{s \rightarrow \infty} h(s)=\lim _{|x| \rightarrow \infty} g(x)=l_{\infty} \in(0, \infty)$

$\left(f_{5}\right) f(x, s) \geq \lim _{|x| \rightarrow \infty} f(x, s)=h(s)$ para todo $x \in \mathbb{R}^{N}, s \in \mathbb{R}^{+}$e $f(x, s)>h(s)$ para $x \in \omega, s \in \mathbb{R}^{+}$, onde $\omega \subset \mathbb{R}^{N}$ é um conjunto de medida positiva. 
Observação 2.1. Temos que $g \in C\left(\mathbb{R}^{N}\right) \cap L^{\infty}\left(\mathbb{R}^{N}\right)$. De fato, como

$$
\lim _{|x| \rightarrow \infty} g(x)=l_{\infty}
$$

da definição de limite, temos que $\forall \varepsilon>0$, existe $M>0$, tal que

$$
\left|g(x)-l_{\infty}\right| \leq \varepsilon, \text { para todo }|x|>M
$$

Assim, $g(x)<l_{\infty}+\varepsilon$, para todo $|x|>M$. Por outro lado, como $g \in C\left(\mathbb{R}^{N}, \mathbb{R}^{+}\right)$, temos que $g(\bar{x})=$ $\max _{x \in B_{M}} g(x)$, para algum $\bar{x} \in B_{M}$. Logo $g \in C\left(\mathbb{R}^{N}\right) \cap L^{\infty}\left(\mathbb{R}^{N}\right)$. Analogamente, $h \in C\left(\mathbb{R}^{+}\right) \cap L^{\infty}\left(\mathbb{R}^{+}\right)$. Por $\left(f_{2}\right)$, ainda temos que

$$
0 \leq f(x, s) \leq g(x)<\infty
$$

Além disso, por um argumento de regularidade que faremos a seguir, a solução $u \in H^{1}\left(\mathbb{R}^{N}\right)$ de $\left(P_{\lambda}\right)$ é tal que $u$ está em $W^{2, p}\left(\mathbb{R}^{N}\right) \cap C^{1}\left(\mathbb{R}^{N}\right)$, para todo $p \geq 2$, de modo que

$$
\lim _{|x| \rightarrow \infty} u(x)=0 \quad \text { e } \quad \lim _{|x| \rightarrow \infty} \nabla u(x)=0
$$

De fato, de acordo com Stuart e Zhou [18], temos o seguinte lema:

Lema 2.1. (a) Para cada função $h \in L^{p}\left(\mathbb{R}^{N}\right), 1 \leq p \leq \infty$, existe um única função $u \in L^{p}\left(\mathbb{R}^{N}\right)$, tal que $u=T h$, satisfazendo a equação $-\Delta u+u=h$ em $\mathbb{R}^{N}$;

(b) Seja $h \in L^{p}\left(\mathbb{R}^{N}\right)$ para algum $p$ com $1 \leq p \leq \infty$. Então, Th $\in L^{p}\left(\mathbb{R}^{N}\right)$ e $|T h|_{p} \leq|h|_{p}$. Caso $p \in(1, \infty)$, nós ainda temos que Th $\in W^{2, p}\left(\mathbb{R}^{N}\right)$ e existe uma constante $C(p, N)$ tal que:

$$
\|T h\|_{W^{2, p}\left(\mathbb{R}^{N}\right)} \leq C(p, N)|h|_{p} .
$$

Demonstração. Segue da Proposição 27, Capítulo II de Dautray e Lions [7] combinado com a estimativa de Calderon-Zygmund $\left|\partial_{i} \partial_{j} u\right|_{p} \leq A(p, N)|\Delta u|_{p}$ (Proposição 3 do Capítulo 3 de Stein [17]).

Por outro lado, como $0 \leq f(x, s) \leq g(x) \leq\|g\|_{\infty}<\infty, \forall x, s \geq 0$, temos que

$$
\|[\lambda+1+f(x, u)] u\|_{p} \leq\left(|\lambda+1|+\|g\|_{\infty}\right)\|u\|_{p}, \quad \forall p \geq 1
$$

Defina $\tilde{f}(x)=(-\lambda+1+f(x, u)) u$, em $\mathbb{R}^{N}$, então temos que $\tilde{f} \in L^{p}\left(\mathbb{R}^{N}\right)$, para $2 \leq p \leq \frac{2 N}{N-2}$, e $u \in L^{p}\left(\mathbb{R}^{N}\right)$ satisfaz

$$
\tilde{f}(x)=(-\lambda+1+f(x, u)) u=u-\lambda u+f(x, u) u=u-\Delta u, \quad \text { em } \quad \mathbb{R}^{N} .
$$

Pelo Lema $2.1(a)$, temos $u=T \tilde{f}$ e por $(b)$ temos $u \in W^{2, p}\left(\mathbb{R}^{N}\right)$, para $2 \leq p \leq \frac{2 N}{N-2}$. Portanto $u$, e consequentemente $\tilde{f}$, pertence a $L^{p}\left(\mathbb{R}^{N}\right)$ para $2 \leq p \leq \infty$ se $N=6$ e $2 \leq p \leq \frac{2 N}{N-6}$ se $N>6$. Um argumento de bootstrap padrão completa a prova de que $u \in W^{2, p}\left(\mathbb{R}^{N}\right), p \geq 2$.

A segunda parte é verdadeira para todo elemento $u \in W^{2, p}\left(\mathbb{R}^{N}\right) \cap C^{1}\left(\mathbb{R}^{N}\right)$ desde que $p>N$. Além disso, como $u(x) \geq 0$, em quase todo ponto de $\mathbb{R}^{N}$, então para qualquer solução de

$$
-\Delta u+\lambda u=f(x, u) u \geq 0, \quad \lambda>0,
$$


o Princípio do Máximo Forte (Teorema 8.19 de Gilbarg e Trudinger [11]) nos mostra que $u(x)>0$ para todo $x \in \mathbb{R}^{N}$.

Ao longo deste trabalho assumiremos, sem perda de generalidade, que $f(x, s)$ e $h(s)$ são definidos para todo $s \in \mathbb{R}$ e $f(x, s)=h(s)=0$ para $s \leq 0$. Nós vamos encontrar soluções positivas de $\left(P_{\lambda}\right)$ como pontos críticos do funcional energia correspondente:

$$
I_{\lambda}(u)=\frac{1}{2}\|u\|_{\lambda}^{2}-\int F(x, u) d x, \quad u \in H^{1}\left(\mathbb{R}^{N}\right),
$$

onde

$$
F(x, s):=\int_{0}^{s} f(x, t) t d t
$$

uma vez que uma solução fraca do problema $\left(P_{\lambda}\right)$ é uma função $u \in H^{1}\left(\mathbb{R}^{N}\right)$ tal que

$$
\int(\nabla u \nabla v+\lambda u v) d x=\int f(x, u) u v d x, \text { para todo } v \in C_{0}^{\infty}\left(\mathbb{R}^{N}\right) .
$$

Além disso, temos que

$$
\|u\|_{\lambda}^{2}:=\int|\nabla u|^{2} d x+\lambda \int|u|^{2} d x
$$

define uma norma equivalente a norma usual de $H^{1}\left(\mathbb{R}^{N}\right)$, a saber, $\|u\|_{H}^{2}=\int|\nabla u|^{2} d x+\int|u|^{2} d x$. De fato, a norma $\|\cdot\|_{\lambda}$ provém do produto interno

$$
\langle u, v\rangle_{\lambda}:=\int \nabla u \nabla v d x+\lambda \int u v d x
$$

Vamos provar que $\langle\cdot, \cdot\rangle_{\lambda}$ é produto interno:

(i)

$$
\begin{aligned}
\langle u, v\rangle_{\lambda} & =\int \nabla u \nabla v d x+\lambda \int u v d x \\
& =\int \nabla v \nabla u d x+\lambda \int v u d x \\
& =\langle v, u\rangle_{\lambda} ;
\end{aligned}
$$

(ii)

$$
\begin{aligned}
\langle\alpha u, v\rangle_{\lambda} & =\int \nabla \alpha u \nabla v d x+\lambda \int \alpha u v d x \\
& =\alpha \int \nabla u \nabla v d x+\lambda \alpha \int u v d x \\
& =\alpha\left(\int \nabla u \nabla v d x+\lambda \int u v d x\right) \\
& =\alpha\langle u, v\rangle_{\lambda} ;
\end{aligned}
$$


(iii)

$$
\begin{aligned}
\langle u+v, w\rangle_{\lambda} & =\int \nabla(u+v) \nabla w d x+\lambda \int(u+v) w d x \\
& =\int(\nabla u \nabla w+\nabla v \nabla w) d x+\lambda \int(u w+v w) d x \\
& =\left(\int \nabla u \nabla w d x+\lambda \int u w d x\right)+\left(\int \nabla v \nabla w d x+\lambda \int v w d x\right) \\
& =\langle u, w\rangle_{\lambda}+\langle v, w\rangle_{\lambda} ;
\end{aligned}
$$

$(i v)$

$$
\langle u, u\rangle_{\lambda}=\int|\nabla u|^{2} d x+\lambda \int|u|^{2} d x \geq 0, \text { já que } \lambda>0 .
$$

Além disso,

$$
\min \{1, \lambda\}\|u\|_{H}^{2} \leq\|u\|_{\lambda}^{2} \leq \max \{1, \lambda\}\|u\|_{H}^{2},
$$

logo as normas são equivalentes.

Vale notar que como $\lim _{s \rightarrow 0} f(x, s)=0$, então para $s$ suficientemente pequeno, temos

$$
\begin{aligned}
& |f(x, s)|<\varepsilon \quad \Rightarrow \\
& -\varepsilon<f(x, s) \quad<\varepsilon \quad \Rightarrow \\
& -\varepsilon s<f(x, s) s \quad<\varepsilon s \quad \Rightarrow \\
& -\varepsilon \frac{s^{2}}{2}<F(x, s) \quad<\varepsilon \frac{s^{2}}{2} \quad \Rightarrow \\
& |F(x, s)|<\varepsilon \frac{s^{2}}{2},
\end{aligned}
$$

e como $\lim _{|x| \rightarrow \infty, s \rightarrow \infty} f(x, s)=l_{\infty}$, então para $|x|$ e $s$ suficientemente grandes, temos

$$
\begin{aligned}
\left|f(x, s)-l_{\infty}\right| & <\varepsilon \quad \Rightarrow \\
f(x, s) & <\varepsilon+l_{\infty} \Rightarrow \\
f(x, s) s & <\varepsilon s+s l_{\infty}
\end{aligned}
$$

e integrando a última desigualdade acima, temos que

$$
F(x, s)<\varepsilon \frac{s^{2}}{2}+\frac{s^{2}}{2} l_{\infty}=\left(\frac{\varepsilon}{2}+\frac{l_{\infty}}{2}\right) s^{2},
$$

assim, $0 \leq F(x, s) \leq C\left(s^{+}\right)^{2}$, para todo $x \in \mathbb{R}^{N}$ e $s \in \mathbb{R}$.

Como pretendemos aplicar o Teorema do Passo da Montanha com condição de Cerami, precisamos saber agora da regularidade do nosso funcional. 


\subsection{Regularidade do Funcional $I_{\lambda}$}

Vamos mostrar que $I_{\lambda}$ é um funcional de classe $C^{1}$ em $H^{1}\left(\mathbb{R}^{N}\right)$. Para isso, precisamos das seguintes definições:

Definição 2.1. Dado um espaço de Banach $X$ e um funcional $I: X \rightarrow \mathbb{R}$, dizemos que I possui Derivada de Fréchet no ponto $u \in X$ quando existe um funcional linear $T \in X^{*}$ tal que

$$
\lim _{\|v\| \rightarrow 0} \frac{I(u+v)-I(u)-T u}{\|v\|}=0 .
$$

A derivada de Fréchet no ponto u, quando existe, é única. Vamos denotá-la por $I^{\prime}(u)$.

Definição 2.2. Se $A$ é um aberto de $X$, dizemos que $I$ é de classe $C^{1}$ em $A$ ou que $I \in C^{1}(A, \mathbb{R})$, quando a derivada de Fréchet de I existe em todo ponto $u \in A$ e a aplicação $I^{\prime}: A \rightarrow X^{*}$ é continua.

Definição 2.3. Dado um espaço de Banach $X$ e um funcional $I: X \rightarrow \mathbb{R}$, dizemos que I possui Derivada de Gateaux no ponto $u \in X$ quando existe um funcional linear $T \in X^{*}$ tal que

$$
\lim _{t \rightarrow 0} \frac{I(u+t v)-I(u)-T v}{t}=0, \quad \text { para todo } v \in X .
$$

A derivada de Gateaux no ponto u, quando existe, é unica. Vamos denotá-la por DI $(u)$.

Observação 2.2. I é de classe $C^{1}$ em A quando a derivada de Gateaux de I em A existe e o operador $D I: X \rightarrow X^{*}$ existe e é contínuo.

Lema 2.2. O funcional $I_{\lambda}$ definido em (2.1) é de classe $C^{1}$.

Demonstração. Já conhecemos as estimativas

$$
\begin{aligned}
f(x, s) s & <\varepsilon s+s l_{\infty} \quad \mathrm{e} \\
F(x, s) & <\left(\frac{\varepsilon}{2}+\frac{l_{\infty}}{2}\right) s^{2}=C\left(s^{+}\right)^{2}
\end{aligned}
$$

vamos usá-las nesta demonstração.

Considere

$$
I_{\lambda}(u)=\frac{1}{2}\|u\|_{\lambda}^{2}-\int F(x, s) d x
$$

e vamos mostrar que $I_{\lambda}$ é de classe $C^{1}$ com

$$
I_{\lambda}^{\prime}(u) v=\int|\nabla u \nabla v| d x+\lambda \int u v d x-\int f(x, u) u v d x .
$$

Considere $I_{\lambda}(u)=J_{0}(u)-J_{1}(u)$, onde

$$
\begin{aligned}
J_{0}(u) & :=\frac{1}{2}\|u\|_{\lambda}^{2}=\frac{1}{2}\left[\int|\nabla u|^{2} d x+\lambda \int|u|^{2} d x\right] \quad \mathrm{e} \\
J_{1}(u) & :=\int F(x, s) d x
\end{aligned}
$$


e vamos mostrar que $J_{0}$ e $J_{1}$ são classe $C^{1}$ com

$$
\begin{aligned}
J_{0}^{\prime}(u) v & =\int|\nabla u \nabla v| d x+\lambda \int u v d x=\langle u, v\rangle_{\lambda} \\
J_{1}^{\prime}(u) v & =\int f(x, u) u v d x
\end{aligned}
$$

Inicialmente, vamos calcular a derivada de Gateaux $D J_{0}$ :

$$
\begin{aligned}
\frac{J_{0}(u+t v)-J_{0}(u)}{t} & =\frac{1}{t}\left[\frac{1}{2} \int|\nabla(u+t v)|^{2} d x+\frac{\lambda}{2} \int(u+t v)^{2} d x-\frac{1}{2} \int|\nabla u|^{2} d x-\frac{\lambda}{2} \int|u|^{2} d x\right] \\
& =\frac{1}{2 t}\left[\int \nabla(u+t v) \nabla(u+t v) d x-\int|\nabla u|^{2} d x+\lambda\left(\int(u+t v)(u+t v) d x-\int|u|^{2} d x\right)\right] \\
& =\frac{1}{2 t}\left[2 t \int \nabla u \nabla v d x+t^{2} \int|\nabla v|^{2} d x+\lambda\left(2 t \int u v d x+t^{2} \int v^{2} d x\right)\right] \\
& =\int \nabla u \nabla v d x+\frac{t}{2} \int|\nabla v|^{2} d x+\lambda \int u v d x+\frac{\lambda t}{2} \int|v|^{2} d x
\end{aligned}
$$

$\log O$

$$
\begin{aligned}
D J_{0}(u) v & =\lim _{t \rightarrow 0} \frac{J_{0}(u+t v)-J_{0}(u)}{t} \\
& =\int \nabla u \nabla v d x+\lambda \int u v d x \\
& =\langle u, v\rangle_{\lambda} .
\end{aligned}
$$

Vamos mostrar agora que $D J_{0}$ é contínua. Seja $\left(u_{n}\right) \subset H^{1}\left(\mathbb{R}^{N}\right)$ tal que $u_{n} \rightarrow u$ em $H^{1}\left(\mathbb{R}^{N}\right)$ e tome $v \in H^{1}\left(\mathbb{R}^{N}\right)$ com $\|v\|_{\lambda} \leq 1, \operatorname{logo}$

$$
\begin{aligned}
\left|\left[D J_{0}\left(u_{n}\right)-D J_{0}(u)\right] v\right| & =\left|\left\langle u_{n}, v\right\rangle_{\lambda}-\langle u, v\rangle_{\lambda}\right| \\
& =\left|\left\langle u_{n}-u, v\right\rangle_{\lambda}\right| \\
& \leq\left\|u_{n}-u\right\|_{\lambda}\|v\|_{\lambda} \\
& \leq\left\|u_{n}-u\right\|_{\lambda} .
\end{aligned}
$$

Obtemos então

$$
\left\|D J_{0}\left(u_{n}\right)-D J_{0}(u)\right\|_{H^{*}}:=\sup _{\|v\|_{\lambda} \leq 1}\left|\left[D J_{0}\left(u_{n}\right)-D J_{0}(u)\right] v\right| \leq\left\|u_{n}-u\right\|_{\lambda} \rightarrow 0
$$

Pela Observação 2.2, temos que $D J_{0}=J_{0}^{\prime}$ é de classe $C^{1}$ e $J_{0}^{\prime}(u) v=\langle u, v\rangle_{\lambda}$.

Vamos calcular agora a derivada de Gateaux $D J_{1}$. Para isso, considere $h:[0,1] \rightarrow \mathbb{R}$ dado por $h(s)=F(x, u+s t v)$ onde $t \in \mathbb{R} \operatorname{com} 0<|t|<1, x \in \mathbb{R}^{N}$ e $u, v \in H^{1}\left(\mathbb{R}^{N}\right)$. Observe que

$$
\begin{aligned}
h^{\prime}(s) & =f(x, u+s t v)(u+s t v) t v \\
h(1) & =F(x, u+t v) \\
h(0) & =F(x, u) .
\end{aligned}
$$

Como $h$ é contínua em $[0,1]$ e diferenciável em $(0,1)$, segue do teorema do valor médio que existe 
$\gamma \in(0,1)$ tal que

$$
h(1)-h(0)=h^{\prime}(\gamma)
$$

de onde concluímos que

$$
\left|\frac{F(x, u+t v)-F(x, u)}{t}\right|=|f(x, u+\gamma t v)(u+\gamma t v) v| .
$$

Da condição de crescimento de $f$ dado em (2.2), obtemos

$$
\begin{aligned}
|f(x, u+\gamma t v)(u+\gamma t v) v| & <|\varepsilon(u+\gamma t v) v|+\left|(u+\gamma t v) v l_{\infty}\right| \\
& <\varepsilon\left|u v+v^{2}\right|+\left|u v+v^{2}\right| l_{\infty} \\
& =C_{\varepsilon}\left|u v+v^{2}\right| \in L^{1}\left(\mathbb{R}^{N}\right) .
\end{aligned}
$$

Tomando uma sequência $\left|t_{n}\right| \rightarrow 0$, usamos o Teorema 1.10 da Convergência Dominada de Lebesgue e obtemos

$$
\begin{aligned}
\lim _{t \rightarrow 0} \frac{J_{1}(u+t v)-J_{1}(u)}{t} & =\lim _{t \rightarrow 0} \frac{\int F(x, u+t v) d x-\int F(x, u) d x}{t} \\
& =\lim _{n \rightarrow \infty} \int f\left(x, u+\gamma t_{n} v\right)\left(u+\gamma t_{n} v\right) v d x \\
& =\int f(x, u) u v d x .
\end{aligned}
$$

Portanto

$$
D J_{1}(u) v=\int f(x, u) u v d x
$$

Vamos agora mostrar que $D J_{1}$ é contínuo. Seja $\left(u_{n}\right) \subset H^{1}\left(\mathbb{R}^{N}\right)$ tal que $u_{n} \rightarrow u$ em $H^{1}\left(\mathbb{R}^{N}\right)$. Das imersões contínuas de Sobolev, temos

$$
u_{n} \rightarrow u \text { em } L^{r}\left(\mathbb{R}^{N}\right), \text { com } 1 \leq r \leq 2^{*}, \text { para } N \geq 3 .
$$

Usando o Teorema 1.13, existe $\left(u_{j}\right) \subset\left(u_{n}\right)$ e $g \in L^{r}\left(\mathbb{R}^{N}\right)$ tal que

$$
\begin{aligned}
u_{j}(x) & \rightarrow u(x), \text { em quase todo ponto de } \mathbb{R}^{N} \text { e } \\
\left|u_{j}(x)\right| & \leq g(x), \text { em quase todo ponto de } \mathbb{R}^{N} .
\end{aligned}
$$

Pela continuidade de $f$, temos que

$$
\left|f\left(x, u_{j}(x)\right) u_{j}(x)-f(x, u(x)) u(x)\right|^{p / p-1} \rightarrow 0, \text { em quase todo ponto de } \mathbb{R}^{N},
$$

e da condição (2.2), obtemos

$$
\begin{aligned}
\left|f\left(x, u_{j}(x)\right) u_{j}(x)-f(x, u(x)) u(x)\right|^{p / p-1} & \leq C\left[\left|f\left(x, u_{j}(x)\right) u_{j}(x)\right|^{p / p-1}+|f(x, u(x)) u(x)|^{p / p-1}\right] \\
& \leq C\left[\varepsilon u_{j}+u_{j} l_{\infty}+\varepsilon u+u l_{\infty}\right] \\
& \leq C_{\varepsilon} g(x) \in L^{1}\left(\mathbb{R}^{N}\right) .
\end{aligned}
$$


Do Teorema 1.9 da Convergência Monótona de Lebesgue, temos que

$$
f\left(x, u_{j}(x)\right) u_{j}(x) \rightarrow f(x, u(x)) u(x), \quad \text { em } L^{\frac{p}{p-1}}\left(\mathbb{R}^{N}\right) .
$$

Assim para todo $v \in H^{1}\left(\mathbb{R}^{N}\right)$ com $\|v\|_{\lambda} \leq 1$, obtemos

$$
\left|\left[D J_{1}\left(u_{j}\right)-D J_{1}(u)\right] v\right|=\int\left[f\left(x, u_{j}\right) u_{j}-f(x, u) u\right] v d x
$$

e como $\frac{p}{p-1}$ e $p$ são expoentes conjugados, então da desigualdade de Hölder, chegamos em

$$
\left|\left[D J_{1}\left(u_{j}\right)-D J_{1}(u)\right] v\right| \leq\left\|f\left(x, u_{j}\right) u_{j}-f(x, u) u\right\|_{\frac{p}{p-1}}\|v\|_{p}
$$

e das imersões contínuas de Sobolev, obtemos

$$
\begin{aligned}
\left|\left[D J_{1}\left(u_{j}\right)-D J_{1}(u)\right] v\right| & \leq C\left\|f\left(x, u_{j}\right) u_{j}-f(x, u) u\right\|_{\frac{p}{p-1}}\|v\|_{\lambda} \\
& \leq C\left\|f\left(x, u_{j}\right) u_{j}-f(x, u) u\right\|_{\frac{p}{p-1}} .
\end{aligned}
$$

Portanto

$$
\begin{aligned}
\left\|D J_{1}\left(u_{j}\right)-D J_{1}(u)\right\| & :=\sup _{\|v\|_{\lambda} \leq 1}\left|\left[D J_{1}\left(u_{j}\right)-D J_{1}(u)\right] v\right| \\
& \leq\left\|f\left(x, u_{j}\right) u_{j}-f(x, u) u\right\|_{\frac{p}{p-1}}
\end{aligned}
$$

o que implica em

$$
\lim _{j \rightarrow \infty} D J_{1}\left(u_{j}\right)=D J_{1}(u)
$$

e, novamente pela Observação 2.2, concluímos que $D J_{1}(u)=J_{1}^{\prime}(u)$ é contínuo e temos o resultado.

Além disso, os pontos críticos de $I_{\lambda}$ são soluções fracas de $\left(P_{\lambda}\right)$. Em particular, se $u$ é um ponto crítico de $I_{\lambda}$, então definindo $u^{+}=\max \{0, u\}$ e $u^{-}=\min \{0, u\}$ temos que $u=u^{+}+u^{-}, u^{+} u^{-}=0$ e $\nabla u^{+} \nabla u^{-}=0$, logo, lembrando que $f(x, u)=0$ para $u \leq 0$, temos

$$
\begin{aligned}
0=I_{\lambda}^{\prime}(u)\left(u^{-}\right) & =\int \nabla u \cdot \nabla u^{-} d x+\lambda \int u u^{-} d x-\int f(x, u) u u^{-} d x \\
& =\int\left|\nabla u^{-}\right|^{2} d x+\lambda \int\left|u^{-}\right|^{2} d x=\left\|u^{-}\right\|_{\lambda}^{2} .
\end{aligned}
$$

Portanto obtemos $u=u^{+} \geq 0$.

Vamos apresentar agora nossa principal ferramenta e a contemplação de uma de suas hipóteses pelo nosso funcional $I_{\lambda}$.

\subsection{Condição de Cerami}

A fim de estabelecer a existência de um ponto crítico não nulo de $I_{\lambda}$, usaremos o Teorema do Passo da Montanha com a condição de Cerami.

Teorema 2.1. Seja $H$ um espaço de Hilbert $e I \in C^{1}(H, \mathbb{R})$ satisfazendo as seguintes condições: 
(a) $\exists \rho, \alpha>0$ tal que $I(u) \geq 0$ para $\|u\| \leq \rho$ e $I(u) \geq \alpha$ para $\|u\|=\rho$;

(b) $I(0)=0$ e existe $e \in H$ com $\|e\|>\rho$ tal que $I(e)<0$.

Seja $\Gamma=\{\gamma \in C([0,1], H) \mid \gamma(0)=0, I(\gamma(1))<0\}$ e defina

$$
c:=\inf _{\gamma \in \Gamma} \sup _{0 \leq t \leq 1} I(\gamma(t))
$$

Se, além disso, o funcional I satisfaz a condição de compacidade de Cerami no nível c, então existe $u \in H$ tal que $I(u)=c \geq \alpha$ e $I^{\prime}(u)=0$.

Recordamos que $I \in C^{1}(H, \mathbb{R})$ satisfaz a condição de Cerami no nível $c \in \mathbb{R}$, que chamaremos de $(C e)_{c}$, se qualquer sequência $\left(u_{n}\right)$, tal que

$$
I\left(u_{n}\right) \rightarrow c \quad \text { e } \quad\left(1+\left\|u_{n}\right\|_{H}\right)\left\|I^{\prime}\left(u_{n}\right)\right\|_{H^{*}} \rightarrow 0
$$

possui uma subsequência convergente.

No resto deste capítulo, vamos mostrar que o funcional $I_{\lambda}$ definido em (2.1) satisfaz a condição $(\mathrm{Ce})_{c}$, quando $c \in \mathbb{R}$ é adequadamente restringido, como veremos no Teorema 2.2. No próximo capítulo, consideramos as condições geométricas do Teorema do Passo da Montanha (condições (a) e (b) acima). Para isso, definimos

$$
\Lambda=\inf \left\{\int\left[|\nabla u|^{2}-g(x) u^{2}\right] d x \mid u \in H^{1}\left(\mathbb{R}^{N}\right), \int u^{2} d x=1\right\} .
$$

Como em Berezin e Shubin [4], temos que $\Lambda=\inf \sigma(S)$, onde $\sigma(S)$ é o espectro do operador definido por

$$
S u(x)=-\Delta u(x)-g(x) u(x), \quad D(S)=H^{2}\left(\mathbb{R}^{N}\right) .
$$

Além disso, do fato que o espectro essencial de $S$ é $\sigma_{e s s}(S)=\left[-l_{\infty}, \infty\right)$, temos

$$
-\|g\|_{\infty} \leq \Lambda \leq-l_{\infty}<0
$$

Observação 2.3. Temos que $\sigma_{\text {ess }}(S)$ consiste nos pontos de acumulação $\sigma(S)$ mais os autovalores de $S$ com multiplicidade infinita. Como $\sigma(S)$ é fechado, temos $\sigma_{\text {ess }}(S) \subset \sigma(S)$.

Começamos com dois lemas técnicos que serão usados nas provas do nosso primeiro resultado de compacidade, a Proposição 2.1.

Lema 2.3. Sob hipótese $\left(f_{1}\right)$, assuma que $\lim _{|x| \rightarrow \infty, s \rightarrow \infty} f(x, s)=l_{\infty} \in \mathbb{R}$ e sejam $\left(v_{n}\right) \subset H^{1}\left(\mathbb{R}^{N}\right), \quad\left(t_{n}\right) \subset$ $\mathbb{R}^{+}$sequências satisfazendo

(i) $v_{n} \rightarrow v, v_{n}(x) \rightarrow v(x)$, para algum $v \in H^{1}\left(\mathbb{R}^{N}\right)$;

(ii) $t_{n} \rightarrow \infty e \frac{I_{\lambda}^{\prime}\left(t_{n} v_{n}\right)}{t_{n}} \rightarrow 0$, quando $n \rightarrow \infty$.

Então qualquer sequência $\left(y_{n}\right) \subset \mathbb{R}^{N}$ tal que $\int_{y_{n}+B_{1}} v_{n}^{2} d x \geq \alpha>0$ é necessariamente limitada.

Demonstração. Vamos fazer a prova por contradição. Seja $\left(y_{n}\right) \subset \mathbb{R}^{N}$ uma sequência tal que

$$
\int_{y_{n}+B_{1}} v_{n}^{2} d x \geq \alpha>0
$$


e suponha que exista uma subsequência de $\left(y_{n}\right)$ ainda denotada por $\left(y_{n}\right)$ tal que $\left|y_{n}\right| \rightarrow \infty$. Definimos agora

$$
\tilde{v}_{n}(x)=v_{n}\left(x+y_{n}\right)
$$

e usando mudança de variáveis $z=x+y_{n}$, temos

$$
\begin{aligned}
\left\|\tilde{v}_{n}\right\|_{\lambda}^{2} & =\int\left|\nabla \tilde{v}_{n}(x)\right|^{2} d x+\lambda \int\left|\tilde{v}_{n}(x)\right|^{2} d x \\
& =\int\left|\nabla v_{n}\left(x+y_{n}\right)\right|^{2} d x+\lambda \int\left|v_{n}\left(x+y_{n}\right)\right|^{2} d x \\
& =\int\left|\nabla v_{n}(z)\right|^{2} d z+\lambda \int\left|v_{n}(z)\right|^{2} d z \\
& =\left\|v_{n}\right\|_{\lambda}^{2}
\end{aligned}
$$

e

$$
\int_{B_{1}} \tilde{v}_{n}^{2} d x=\int_{B_{1}} v_{n}^{2}\left(x+y_{n}\right) d x=\int_{B_{1}+y_{n}} v_{n}^{2}(z) d z \geq \alpha
$$

Sabemos que $\left(\tilde{v}_{n}\right)$ é limitada em $H^{1}\left(\mathbb{R}^{N}\right)$, já que $v_{n} \rightarrow v$ em $H^{1}\left(\mathbb{R}^{N}\right)$. Podemos então obter $\tilde{v}$ em $H^{1}\left(\mathbb{R}^{N}\right)$ de modo que, a menos de subsequência,

$$
\begin{aligned}
\tilde{v}_{n} & \rightarrow \tilde{v}, & \text { em } H^{1}\left(\mathbb{R}^{N}\right) ; \\
\tilde{v}_{n}(x) & \rightarrow \tilde{v}(x), & \text { em quase todo ponto de } \mathbb{R}^{N} .
\end{aligned}
$$

Por outro lado, como o operador restrição de $H^{1}\left(\mathbb{R}^{N}\right) \hookrightarrow H^{1}\left(B_{1}\right)$ é contínuo, e a imersão

$$
H^{1}\left(B_{1}\right) \hookrightarrow L^{r}\left(B_{1}\right), \quad 1 \leq r<2^{*}
$$

é compacta, temos que

$$
\tilde{v}_{n} \rightarrow \tilde{v}, \quad \text { em } L^{2}\left(B_{1}\right)
$$

Assim,

$$
\int_{B_{1}} \tilde{v}^{2} d x=\lim _{n \rightarrow \infty} \int_{B_{1}} \tilde{v}_{n}^{2} d x \geq \alpha>0
$$

e portanto $\tilde{v} \neq 0$.

Considere agora $\phi \in C_{0}^{\infty}\left(\mathbb{R}^{N}\right)$, denotando $\phi_{n}(x)=\phi\left(x-y_{n}\right)$ e fazendo a mudança de variáveis $x=z+y_{n}$, obtemos

$$
\begin{aligned}
\frac{I_{\lambda}^{\prime}\left(t_{n} v_{n}\right)}{t_{n}}\left(\phi_{n}\right) & =\int\left(\nabla v_{n}(x) \nabla \phi_{n}(x)+\lambda v_{n}(x) \phi_{n}(x)\right) d x-\int f\left(x, t_{n} v_{n}(x)\right) v_{n}(x) \phi_{n}(x) d x \\
& =\int\left(\nabla v_{n}\left(z+y_{n}\right) \nabla \phi_{n}\left(z+y_{n}\right)+\lambda v_{n}\left(z+y_{n}\right) \phi_{n}\left(z+y_{n}\right)\right) d z \\
& -\int f\left(z+y_{n}, t_{n} v_{n}\left(z+y_{n}\right)\right) v_{n}\left(z+y_{n}\right) \phi_{n}\left(z+y_{n}\right) d z \\
& =\int\left(\nabla \tilde{v}_{n}(z) \nabla \phi(z)+\lambda \tilde{v}_{n}(z) \phi(z)\right) d z-\int f\left(z+y_{n}, t_{n} v_{n}\left(z+y_{n}\right)\right) v_{n}\left(z+y_{n}\right) \phi(z) d z
\end{aligned}
$$


Por $(i i)$, temos

$$
\frac{I_{\lambda}^{\prime}\left(t_{n} v_{n}\right)}{t_{n}} \phi_{n}=o(1)
$$

logo vamos analisar a convergência de

$$
\int\left(\nabla \tilde{v}_{n} \cdot \nabla \phi+\lambda \tilde{v}_{n} \phi\right) d z
$$

e de

$$
\int f\left(z+y_{n}, t_{n} v_{n}\left(z+y_{n}\right)\right) v_{n}\left(z+y_{n}\right) \phi(z) d z .
$$

Uma vez que $\tilde{v}_{n} \rightarrow \tilde{v}$, obtemos

$$
\int\left(\nabla \tilde{v}_{n} \cdot \nabla \phi+\lambda \tilde{v}_{n} \phi\right) d z \rightarrow \int(\nabla \tilde{v} \nabla \phi+\lambda \tilde{v} \phi) d z .
$$

Vamos agora analisar a convergência de $\int f\left(z+y_{n}, t_{n} v_{n}\left(z+y_{n}\right)\right) v_{n}\left(z+y_{n}\right) \phi(z) d z$. Primeiramente note que estamos assumindo $f(x, s)=0$ para $s \leq 0$ e assim $f\left(z+y_{n}, t_{n} v_{n}\left(z+y_{n}\right)\right) v_{n}\left(z+y_{n}\right) \geq 0$. Agora, como $\tilde{v}_{n} \rightarrow \tilde{v}$, em quase todo ponto de $\mathbb{R}^{N}$, então $\left|t_{n} \tilde{v}_{n}\right| \rightarrow \infty$ e assim, utilizando $\lim _{|x| \rightarrow \infty, s \rightarrow \infty} f(x, s)=l_{\infty}$, chegamos em

$$
f\left(z+y_{n}, t_{n} v_{n}\left(z+y_{n}\right)\right) v_{n}\left(z+y_{n}\right) \phi(z) \rightarrow l_{\infty} \tilde{v}^{+}(z) \phi(z),
$$

em quase todo ponto de $\mathbb{R}^{N}$, quando $n \rightarrow \infty$.

Temos, como antes, que $\tilde{v}_{n} \rightarrow \tilde{v}$ em $L^{1}\left(B_{R}\right)$, onde $\operatorname{supp}(\phi) \subset B_{R}$. Lembramos agora do Teorema 1.13, que nos diz que se tivermos uma sequência $\left(u_{n}\right) \subset L^{p}(\Omega)$ com $\Omega$ limitado, e $u \in L^{p}(\Omega)$ tal que $\left\|u_{n}-u\right\|_{L^{p}(\Omega)} \rightarrow 0$, então existe uma subsequência $u_{n_{k}}$ tal que $u_{n_{k}}(x) \rightarrow u(x)$, em quase todo ponto de $\Omega$ e além disso, existe uma função $r \in L^{p}(\Omega)$ tal que $\left|u_{n_{k}}(x)\right| \leq r(x)$, em quase todo ponto de $\Omega$. No nosso caso, existe $r \in L^{1}\left(B_{R}\right)$ tal que, a menos de subsequência,

$$
\left|\tilde{v}_{n}(x)\right| \leq r(x), \text { em } B_{R}
$$

e

$$
\left|f\left(z+y_{n}, t_{n} v_{n}\left(z+y_{n}\right)\right) v_{n}\left(z+y_{n}\right) \phi(z)\right| \leq C r(z), \quad \forall z \in \mathbb{R}^{N} \text { e } C=l_{\infty}\|\phi\|_{\infty} .
$$

Como $\left|y_{n}\right| \rightarrow \infty$ e $t_{n} \rightarrow \infty$, segue do Teorema 1.10 da Convergência Dominada de Lebesgue que

$$
\int f\left(z+y_{n}, t_{n} v_{n}\left(z+y_{n}\right)\right) v_{n}\left(z+y_{n}\right) \phi(z) d z \rightarrow \int l_{\infty} \tilde{v}^{+}(z) \phi(z) d z .
$$

Assim, concluímos que

$$
\int(\nabla \tilde{v} \nabla \phi+\lambda \tilde{v} \phi) d z-\int l_{\infty} \tilde{v}^{+}(z) \phi(z) d z=0, \quad \forall \phi \in C_{0}^{\infty}\left(\mathbb{R}^{N}\right)
$$

Desta forma, $\tilde{v}$ é uma solução fraca não trivial do problema

$$
-\Delta \tilde{v}=\left(l_{\infty}-\lambda\right) \tilde{v}, \quad \text { em } \mathbb{R}^{N} .
$$

Por outro lado, sabemos que se $u \in H^{1}\left(\mathbb{R}^{N}\right)$ for uma solução fraca do problema limite

$$
-\Delta u+\lambda u=l_{\infty} u, \quad \lambda>0
$$


então $u \in H^{2}\left(\mathbb{R}^{N}\right)$ e portanto, para nosso caso particular, teremos $\tilde{v} \in H^{2}\left(\mathbb{R}^{N}\right)$. Mas isso gera uma contradição, pois se $\lambda \in \mathbb{R}$ e $u \in H^{2}\left(\mathbb{R}^{N}\right)$ é solução do problema do autovalor

$$
-\Delta u=\lambda u, \quad \text { em } \mathbb{R}^{N}
$$

então $u=0$. Portanto, a contradição vem de supormos que $\left(y_{n}\right)$ não é limitado, logo $\left(y_{n}\right)$ deve ser limitado e temos o resultado.

Eis nosso segundo principal lema desta seção.

Lema 2.4. Sob a hipótese $\left(f_{1}\right)$, assuma que $\lim _{s \rightarrow \infty} f(x, s)=g(x) \in C\left(\mathbb{R}^{N}, \mathbb{R}^{+}\right), \liminf _{|x| \rightarrow \infty} g(x)=l_{\infty} \in \mathbb{R} e$ sejam $\left(v_{n}\right) \subset H^{1}\left(\mathbb{R}^{N}\right),\left(t_{n}\right) \subset \mathbb{R}^{+}$sequências satisfazendo

(i) $\quad v_{n} \rightarrow v, v_{n}(x) \rightarrow v(x)$, para algum $v \in H^{1}\left(\mathbb{R}^{N}\right)$;

(ii) $\frac{I_{\lambda}^{\prime}\left(t_{n} v_{n}\right)}{t_{n}} \rightarrow 0$, quando $n \rightarrow \infty$;

Se $t_{n} \rightarrow \infty$, então $v=0$ ou $\lambda=-\Lambda$, onde $\Lambda$ é dado por (2.4).

Demonstração. Vamos assumir que $v \neq 0$ e concluir que necessariamente $\lambda=-\Lambda$. De fato, usando (ii) obtemos, para $\phi \in C_{0}^{\infty}\left(\mathbb{R}^{N}\right)$ arbitrário, que

$$
\int\left(\nabla v_{n} . \nabla \phi+\lambda v_{n} \phi\right) d x-\int f\left(x, t_{n} v_{n}\right) v_{n} \phi d x=\frac{I_{\lambda}^{\prime}\left(t_{n} v_{n}\right)}{t_{n}} \phi=o(1)
$$

Como $v_{n} \rightarrow v$ em $H^{1}\left(\mathbb{R}^{N}\right)$, obtemos

$$
\int\left(\nabla v_{n} \nabla \phi+\lambda v_{n} \phi\right) d x \rightarrow \int(\nabla v \nabla \phi+\lambda v \phi) d x .
$$

Como feito no Lema 2.3, tem-se

$$
f\left(x, t_{n} v_{n}(x)\right) v_{n}(x) \phi(x) \rightarrow g(x) v^{+}(x) \phi(x), \text { em quase todo ponto de } \mathbb{R}^{N}
$$

e

$$
\left|f\left(x, t_{n} v_{n}\right) v_{n} \phi\right| \leq C r
$$

para algum $r \in L^{1}\left(B_{R}\right) \operatorname{com} C=\|g\|_{\infty}\|\phi\|_{\infty}, \forall x \in \mathbb{R}^{N}$, de modo que o Teorema 1.10 da Convergência Dominada de Lebesgue e o fato de que $t_{n} \rightarrow \infty$, nos fornecem

$$
\int f\left(x, t_{n} v_{n}\right) v_{n} \phi d x \rightarrow \int g(x) v^{+} \phi d x .
$$

Assim passando (2.7) ao limite, temos que

$$
\int(\nabla v \nabla \phi+\lambda v \phi) d x-\int g(x) v^{+} \phi d x=0
$$

e considerando $\phi=v^{-}$, obtemos

$$
\left\|v^{-}\right\|_{\lambda}^{2}=\int\left(\nabla v^{-} \nabla v^{-}+\lambda v^{-} v^{-}\right) d x=\int g(x) v^{+} v^{-} d x=0
$$

e segue que $v=v^{+} \geq 0$ é uma solução fraca de $-\Delta v+\lambda v=g(x) v$. Concluímos pelo Princípio do Máximo que $v(x)>0$ para todo $x \in \mathbb{R}^{N}$ e, por regularidade elíptica, que $v \in H^{2}\left(\mathbb{R}^{N}\right)$. Além disso, segue da 
identidade de Green que

$$
\int[-\Delta v+(\lambda-g(x)) v] \phi d x=0, \quad \forall \phi \in C_{0}^{\infty}\left(\mathbb{R}^{N}\right)
$$

Sabemos, agora, do resultado que diz que se $u \in L_{l o c}^{1}(\Omega)$ é tal que $\int_{\Omega} u \phi d x=0, \forall \phi \in C_{0}^{\infty}(\Omega)$, então $u=0$, em quase todo ponto de $\Omega$ e assim, no nosso caso, temos que

$$
-\Delta v+(\lambda-g(x)) v=0, \quad \text { em quase todo ponto de } \mathbb{R}^{N},
$$

desde que $-\Delta v+(\lambda-g(x)) v$ esteja em $L_{l o c}^{1}\left(\mathbb{R}^{N}\right)$. Como $v \in H^{2}\left(\mathbb{R}^{N}\right)$, então $-\Delta v \in L^{2}\left(\mathbb{R}^{N}\right), \operatorname{logo}-\Delta v \in$ $L_{l o c}^{1}\left(\mathbb{R}^{N}\right)$ e, analogamente, $v \in L_{l o c}^{1}\left(\mathbb{R}^{N}\right)$. Além disso, como $-g(x) \leq\|g\|_{\infty}<\infty$, então $-\int_{K} g(x) d x \leq$ $\|g\|_{\infty}|K|<\infty$, já que $K$ é limitado, então teremos

$$
\begin{aligned}
\int_{K}(-\Delta v+(\lambda-g(x)) v) d x & =\int_{K}-\Delta v d x+\lambda \int_{K} v d x-\int_{K} g(x) v d x \\
& \leq \int_{K}-\Delta v d x+\lambda \int_{K} v d x+\|g\|_{\infty} \int_{K} v d x<\infty
\end{aligned}
$$

Ainda por (2.9), concluímos que

$$
S v=-\lambda v
$$

onde $S v=-\Delta v-g(x) v$.

Assim, $v$ é uma autofunção positiva de $S$ com o autovalor $-\lambda$. Tomando $\phi=v$ em (2.8) com $\|v\|_{L^{2}\left(\mathbb{R}^{N}\right)}=1$, obtemos

$$
\int\left(|\nabla v|^{2}-g(x) v^{2}\right) d x=-\lambda
$$

e assim, pela definição de $\Lambda$, tem-se

$$
\Lambda \leq-\lambda
$$

Lembrando também que $\Lambda \leq-l_{\infty}$ por (2.6), vamos considerar dois casos:

Caso 1) $\Lambda<-l_{\infty}$.

Considerando

$$
\begin{aligned}
S_{0}: D\left(S_{0}\right) & \rightarrow L^{2}\left(\mathbb{R}^{N}\right) \\
u & \mapsto S_{0}(u)=-\Delta u+v(x) u,
\end{aligned}
$$

$\operatorname{com} v \in L_{l o c}^{1}\left(\mathbb{R}^{N}\right)$ e $D\left(S_{0}\right)=C_{0}^{\infty}\left(\mathbb{R}^{N}\right) \subset L^{2}\left(\mathbb{R}^{N}\right)$ e se

$$
\liminf _{|x| \rightarrow \infty} v(x)=\lim _{r \rightarrow \infty} \inf _{|x| \rightarrow r} v(x) \geq \alpha
$$

então o operador $S_{0}$ é limitado inferiormente. Além disso, para cada $a^{\prime}<a$ temos que $\sigma\left(S_{0}\right) \cap\left(-\infty, a^{\prime}\right)$ consiste de um número finito de elementos (autovalores de multiplicidade finita) pertencentes a $\sigma_{d}\left(S_{0}\right)$.

Desta forma, para o nosso caso, temos que $S$ possui um número finito de autovalores $\lambda_{1}, \cdots, \lambda_{k}$ ou 
uma sequência $\left(\lambda_{n}\right)$ de autovalores tal que $\lambda_{n} \rightarrow-l_{\infty}$, onde cada $\lambda_{i}$ possui multiplicidade finita para cada $i$.

Lembramos agora do resultado, que pode ser visto em [4], que nos diz que se $T: D(T) \subset H \rightarrow H$ for um operador auto-adjunto, semi limitado inferiormente e $K \subset H$ um subespaço denso no qual $T$ é auto-adjunto e da caracterização variacional dos autovalores, temos que

$$
\lambda_{1}=\inf _{0 \neq x \in K} \frac{\langle T x, x\rangle_{\lambda}}{\langle x, x\rangle_{\lambda}} \quad \text { e } \quad \lambda_{n+1}=\sup _{L \subset K} \inf _{0 \neq x \in K \cap L^{\perp}} \frac{\langle T x, x\rangle_{\lambda}}{\langle x, x\rangle_{\lambda}} .
$$

Logo, temos que $\Lambda$ é o menor autovalor de $S$, ou seja, existe $u_{0} \in H^{1}\left(\mathbb{R}^{N}\right)$ com $\left\|u_{0}\right\|_{L^{2}\left(\mathbb{R}^{N}\right)}=1$, tal que

$$
\int\left(\left|\nabla u_{0}\right|^{2}-g(x) u_{0}^{2}\right) d x=\Lambda
$$

onde $u_{0}$ é a autofunção correspondente ao autovalor $\Lambda$. Usando (2.12), temos que $u_{0} \geq 0$. Lembramos agora do resultado que nos diz que se $u \geq 0$ é solução fraca não trivial de $-\Delta u+\lambda u=g(x) u$, então $u>0$. Obtemos assim que $u_{0}>0$. Logo

$$
\Lambda=-\lambda,
$$

já que, caso contrário, $S$ teria duas autofunções positivas, $v$ e $u_{0}$, associadas a autovalores distintos, o que é um absurdo, pois autofunções associadas a autovalores distintos são ortogonais.

Caso 2) $\Lambda=-l_{\infty}$.

No Caso 2 , temos $\Lambda=-l_{\infty} \leq-\lambda$ e, novamente, mostraremos que $\Lambda=-l_{\infty}=-\lambda$. De fato, se $\Lambda=-l_{\infty}<-\lambda$, definimos $\delta:=\frac{l_{\infty}-\lambda}{2}>0$ e seja $R_{1}>0$, tal que

$$
g(x) \geq l_{\infty}-\delta, \text { para todo }|x| \geq R_{1},
$$

e tome $R_{2}>R_{1}$ suficientemente grande, tal que

$$
0<\mu_{1} \leq \frac{\delta}{2}
$$

onde $\mu_{1}$ é o primeiro autovalor de $-\Delta$ no anel $A=\left\{x\left|R_{1}<\right| x \mid<R_{2}\right\}$ com a condição de contorno de Dirichlet. Seja $\psi$ a autofunção correspondente e, por ter sinal definido, tomamos, sem perda de generalidade, $\psi>0$, e obtemos de (2.10) e (2.13) que

$$
\begin{aligned}
S v & =-\lambda v \Rightarrow \\
-\Delta v-g v & =-\lambda v \Rightarrow \\
\int_{A}-\Delta v \psi d x-\int_{A} g v \psi d x & =-\lambda \int_{A} v \psi d x
\end{aligned}
$$


Logo,

$$
\begin{aligned}
\int_{A}-\Delta v \psi d x & =\int_{A}(g-\lambda) v \psi d x \\
& \geq \int_{A}\left(l_{\infty}-\delta-\lambda\right) v \psi d x \\
& =\int_{A}\left(l_{\infty}-\frac{\left(l_{\infty}-\lambda\right)}{2}-\lambda\right) v \psi d x \\
& =\int_{A} \frac{\left(l_{\infty}-\lambda\right)}{2} v \psi d x \\
& =\delta \int_{A} v \psi d x,
\end{aligned}
$$

ou seja,

$$
\int_{A}(-\Delta v) \psi d x \geq \delta \int_{A} v \psi d x
$$

Por outro lado, como

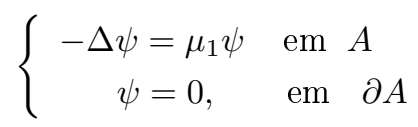

e, pelo Lema 1.1 de Hopf,

$$
\frac{\partial \psi}{\partial n}<0, \quad \text { em } \partial A
$$

(note que estamos usando $L \psi=-\Delta \psi>0$ e que $L$ está na forma divergente), usamos a identidade de Green

$$
\int_{A}(-\Delta v) \psi d x=\int_{A}(-\Delta \psi) v d x+\int_{\partial A} v \frac{\partial \psi}{\partial n} d x-\int_{\partial A} \psi \frac{\partial v}{\partial n} d x
$$

para concluir que

$$
\int_{A}(-\Delta v) \psi d x \leq \int_{A}(-\Delta \psi) v d x=\mu_{1} \int_{A} v \psi d x
$$

e como $0<\mu_{1} \leq \frac{\delta}{2}$, então

$$
\int_{A}(-\Delta v) \psi d x \leq \frac{\delta}{2} \int_{A} v \psi d x
$$

o que contradiz (2.15). Portanto, também devemos ter $\Lambda=-\lambda$ no Caso 2. Assim a prova fica completa.

A seguir apresentaremos uma proposição fundamental para a demonstração da condição de Cerami.

Proposição 2.1. Assuma as condições $\left(f_{1}\right)-\left(f_{4}\right)$ e suponha que $\left(u_{n}\right)$ é uma sequência de Cerami no nivel $c>0$ para o funcional $I_{\lambda}$. Então $\left(u_{n}\right)$ é limitada desde que $\lambda \neq-\Lambda$.

Demonstração. Suponha, por contradição, que $\left(u_{n}\right)$ é uma sequência de Cerami no nível $c>0$ para o funcional $I_{\lambda}$ com $\left\|u_{n}\right\|_{\lambda} \rightarrow \infty$. Defina $v_{n}=2 \sqrt{c}\left(\frac{u_{n}}{\left\|u_{n}\right\|_{\lambda}}\right)$. Então, $\left\|v_{n}\right\|_{\lambda}=2 \sqrt{c}$ e assim $\left(v_{n}\right)$ é limitado.

Vamos agora usar o Teorema 1.6, que nos diz que se $X$ é um espaço de Banach reflexivo e se $\left(u_{n}\right)$ é uma sequência limitada em $X$, então $\left(u_{n}\right)$ possui uma subsequência que converge fracamente. Como $H^{1}\left(\mathbb{R}^{N}\right)$ é um espaço de Banach reflexivo, temos que existe $v \in H^{1}\left(\mathbb{R}^{N}\right)$ tal que

$$
v_{n} \rightarrow v, \text { em } H^{1}\left(\mathbb{R}^{N}\right) \quad \mathrm{e}
$$




$$
v_{n}(x) \rightarrow v(x) \text {, em quase todo ponto de } \mathbb{R}^{N} .
$$

Por outro lado, o operador restrição de $H^{1}\left(\mathbb{R}^{N}\right)$ em $H^{1}\left(B_{R}\right)$ é contínuo e a imersão de $H^{1}\left(B_{R}\right)$ em $L^{p}\left(B_{R}\right), 1 \leq p<2^{*}$ é compacta, logo

$$
v_{n} \rightarrow v, \quad \text { em } \quad L^{p}\left(B_{R}\right)
$$

para todo $R>0$. Em outras palavras, temos que $v_{n} \rightarrow v$ em $L_{l o c}^{p}\left(\mathbb{R}^{N}\right)$, para qualquer $2 \leq p<2^{*}$, onde $2^{*}=\frac{2 N}{N-2}$ se $N \geq 3$ e $2^{*}=\infty$ se $N=1,2$.

Como $\left(u_{n}\right)$ é uma sequência de Cerami no nível $c$, obtemos

$$
\left(1+\left\|u_{n}\right\|_{\lambda}\right)\left\|I_{\lambda}^{\prime}\left(u_{n}\right)\right\| \rightarrow 0
$$

e assim

$$
\left\|u_{n}\right\|_{\lambda}\left\|I_{\lambda}^{\prime}\left(u_{n}\right)\right\| \leq\left(1+\left\|u_{n}\right\|_{\lambda}\right)\left\|I_{\lambda}^{\prime}\left(u_{n}\right)\right\|
$$

logo podemos assumir, sem perda de generalidade, que

$$
\left\|u_{n}\right\|_{\lambda}\left\|I_{\lambda}^{\prime}\left(u_{n}\right)\right\| \leq \frac{1}{n}
$$

Então, $(2.16)$ e $\left(f_{2}\right)$ implicam que

$$
I_{\lambda}\left(\tau u_{n}\right) \leq \frac{1+\tau^{2}}{2 n}+I_{\lambda}\left(u_{n}\right)
$$

para todo $\tau>0$ e $n \in \mathbb{N}$. De fato, considere

$$
F(x, s):=\int_{0}^{s} f(x, \tau) \tau d \tau
$$

e o funcional

$$
I_{\lambda}(u):=\frac{1}{2}\|u\|_{\lambda}^{2}-\int F(x, u(x)) d x
$$

para $u \in H^{1}\left(\mathbb{R}^{N}\right)$.

Seja $\left(u_{n}\right) \subset H^{1}\left(\mathbb{R}^{N}\right)$ tal que $I_{\lambda}\left(u_{n}\right) \rightarrow c,\left\|u_{n}\right\|_{\lambda} \rightarrow \infty$ e $\left\|I_{\lambda}^{\prime}\left(u_{n}\right)\right\|\left\|u_{n}\right\|_{\lambda}<\frac{1}{n}, \operatorname{logo}$

$$
-\frac{1}{n}<I_{\lambda}^{\prime}\left(u_{n}\right) u_{n}=\left\|u_{n}\right\|_{\lambda}^{2}-\int f\left(x, u_{n}\right) u_{n}^{2} d x<\frac{1}{n} .
$$

Defina

$$
\theta(\tau):=\frac{1}{2} \tau^{2} f\left(x, u_{n}\right) u_{n}^{2}-F\left(x, \tau u_{n}\right)
$$

$\log 0$

$$
\theta^{\prime}(\tau)=\tau f\left(x, u_{n}\right) u_{n}^{2}-f\left(x, \tau u_{n}\right) \tau u_{n}^{2}=\tau u_{n}^{2}\left[f\left(x, u_{n}\right)-f\left(x, \tau u_{n}\right)\right]
$$

e como $f$ é não decrescente na segunda coordenada e $\tau u_{n}^{2}>0$, temos que

$$
\begin{cases}\theta^{\prime}(\tau) \geq 0, & 0<\tau \leq 1 \\ \theta^{\prime}(\tau) \leq 0, & \tau \geq 1\end{cases}
$$

portanto $\tau=1$ é o ponto de máximo de $\theta$, para $\tau>0$, $\log o$ 


$$
\theta(\tau) \leq \theta(1)
$$

Por outro lado, de (2.18) e (2.19), temos

$$
\begin{aligned}
I_{\lambda}\left(\tau u_{n}\right) & =\frac{1}{2} \tau^{2}\left\|u_{n}\right\|_{\lambda}^{2}-\int F\left(x, \tau u_{n}\right) d x \\
& <\frac{\tau^{2}}{2}\left[\frac{1}{n}+\int f\left(x, u_{n}\right) u_{n}^{2} d x\right]-\int F\left(x, \tau u_{n}\right) d x \\
& =\frac{\tau^{2}}{2 n}+\int\left[\frac{\tau^{2}}{2} f\left(x, u_{n}\right) u_{n}^{2}-F\left(x, \tau u_{n}\right)\right] d x \\
& \leq \frac{\tau^{2}}{2 n}+\int\left[\frac{1}{2} f\left(x, u_{n}\right) u_{n}^{2}-F\left(x, u_{n}\right)\right] d x .
\end{aligned}
$$

Além disso, por (2.18), temos

$$
I_{\lambda}\left(u_{n}\right)=\frac{1}{2}\left\|u_{n}\right\|_{\lambda}^{2}-\int F\left(x, u_{n}\right) d x \geq \frac{1}{2}\left[-\frac{1}{n}+\int f\left(x, u_{n}\right) u_{n}^{2} d x\right]-\int F\left(x, u_{n}\right) d x
$$

o que implica em

$$
\int\left[\frac{1}{2} f\left(x, u_{n}\right) u_{n}^{2}-F\left(x, u_{n}\right)\right] d x \leq I_{\lambda}\left(u_{n}\right)+\frac{1}{2 n}
$$

e assim

$$
I_{\lambda}\left(\tau u_{n}\right) \leq \frac{\tau^{2}}{2 n}+\frac{1}{2 n}+I_{\lambda}\left(u_{n}\right)=\frac{1+\tau^{2}}{2 n}+I_{\lambda}\left(u_{n}\right)
$$

e provamos $(2.17)$.

Considere $v_{n}=\tau_{n} u_{n}, \operatorname{com} \tau_{n}=\frac{2 \sqrt{c}}{\left\|u_{n}\right\|_{\lambda}} \rightarrow 0, \log \mathrm{O}$

$$
\begin{aligned}
I_{\lambda}\left(v_{n}\right) & =I_{\lambda}\left(\tau_{n} u_{n}\right) \\
& \leq \frac{1+\tau_{n}^{2}}{2 n}+I_{\lambda}\left(u_{n}\right) \\
& \leq I_{\lambda}\left(u_{n}\right)+o(1) \\
& =c+o(1),
\end{aligned}
$$

para $n$ suficientemente grande.

Afirmação: $v \neq 0$.

Para provar essa afirmação, precisamos das seguintes estimativas, que vamos demonstrar logo em seguida

$$
F(x, s) \leq \frac{1}{2} f(x, s) s^{2}
$$

e

$$
\left|f(x, s) s^{2}\right| \leq \varepsilon s^{2}+C(\varepsilon, q) s^{q}
$$

para qualquer $x \in \mathbb{R}^{N}, s \in \mathbb{R}^{+}, \varepsilon>0$ e $2<q \leq 2^{*}$. De fato, como $f(x, s)$ é não decrescente em $s$, segue que

$$
F(x, s)=\int_{0}^{s} f(x, t) t d t \leq f(x, s) \int_{0}^{s} t d t=\frac{1}{2} f(x, s) s^{2}
$$


provando assim a estimativa dada em (2.21). Agora por $\left(f_{1}\right)$, dado $\varepsilon>0$, existe $\delta>0$, tal que

$$
|f(x, s)|<\varepsilon, \quad \text { se } s<\delta,
$$

e por $\left(f_{2}\right)$, existe $K \geq 1$ de modo que

$$
f(x, s)<g(x)+\varepsilon \leq\|g\|_{\infty}+\varepsilon=C_{\varepsilon}, \text { para } s>K .
$$

Assim temos, para $2<q \leq 2^{*}$, que

$$
\begin{gathered}
f(x, s) s^{2}<\varepsilon s^{2}, \quad \text { para } s<\delta \\
f(x, s) s^{2}<C_{\varepsilon} s^{2}<C_{\varepsilon} s^{q}, \quad \text { para } s>K .
\end{gathered}
$$

Definimos agora, para cada $q \in\left(2,2^{*}\right]$

$$
\begin{aligned}
\phi_{q}:[\delta, K] & \rightarrow \mathbb{R} \\
s & \rightarrow \phi_{q}(s)=\frac{f(x, s) s^{2}}{s^{q}} .
\end{aligned}
$$

Logo, $\phi_{q}$ é contínua e assume máximo no compacto $[\delta, K]$, isto é,

$$
\frac{f(x, s) s^{2}}{s^{q}} \leq C(\varepsilon, q), \quad \text { para s pertencente a }[\delta, K],
$$

e está provada a estimativa $(2.22)$.

A fim de provar que $v \neq 0$, consideramos a função concentração de $\left|v_{n}\right|^{2}$, dada por

$$
Q_{n}(t)=\sup _{y \in \mathbb{R}^{N}} \int_{y+B_{t}}\left|v_{n}\right|^{2} d x, \quad t>0 .
$$

Agora, se $\lim _{n \rightarrow \infty} Q_{n}\left(t_{0}\right)=0$, para algum $t_{0}>0$, o Lema 1.2 de Lions nos fornece $v_{n} \rightarrow 0 \in L^{p}\left(\mathbb{R}^{N}\right)$ para qualquer $2<p<2^{*}$. Assim, por (2.21) e (2.22), temos

$$
\begin{aligned}
\int F\left(x, v_{n}\right) d x & \leq \frac{1}{2} \int f\left(x, v_{n}\right) v_{n}^{2} d x \\
& \leq \int\left(\frac{1}{2} \varepsilon\left|v_{n}\right|^{2}+C(\varepsilon, q)\left|v_{n}\right|^{q}\right) d x \\
& =\frac{1}{2} \varepsilon\left\|v_{n}\right\|_{L^{2}\left(\mathbb{R}^{N}\right)}^{2}+C(\varepsilon, q)\left\|v_{n}\right\|_{L^{q}\left(\mathbb{R}^{N}\right)}^{q}=o(1),
\end{aligned}
$$

portanto

$$
I_{\lambda}\left(v_{n}\right)=\frac{1}{2}\left\|v_{n}\right\|_{\lambda}^{2}-\int F\left(x, v_{n}\right) d x \geq 2 c+o(1),
$$

o que contradiz (2.20), já que $c>0$. Portanto, podemos assumir que $\lim _{n \rightarrow \infty} Q_{n}(t)>0, \forall t>0$ e, em particular para $t=1$ temos, a menos de subsequência,

$$
Q_{n}(1)=\sup _{y \in \mathbb{R}^{N}} \int_{y+B_{1}}\left|v_{n}\right|^{2} d x>\alpha>0
$$


para algum $\alpha>0$ e para todo $n \in \mathbb{N}$. Segue, para alguma sequência $\left(y_{n}\right) \subset \mathbb{R}^{N}$, que

$$
\int_{y_{n}+B_{1}}\left|v_{n}\right|^{2} d x \geq \alpha>0
$$

De fato, se

$$
\int_{y_{n}+B_{1}}\left|v_{n}\right|^{2} d x<\alpha
$$

para toda sequência $\left(y_{n}\right) \subset \mathbb{R}^{N}$, então, em particular, para a sequência constante $y_{n}=y$, teremos

$$
Q_{n}(1)=\sup _{y \in \mathbb{R}^{N}} \int_{y+B_{1}}\left|v_{n}\right|^{2} d x<\alpha
$$

o que é um absurdo.

Em vista de (2.16), tomando $t_{n}:=\tau_{n}^{-1}=\frac{\left\|u_{n}\right\|_{\lambda}}{2 \sqrt{c}}$, temos que

$$
\begin{aligned}
t_{n} & \rightarrow \infty, \\
t_{n} v_{n} & =u_{n} \quad \mathrm{e} \\
\left\|I_{\lambda}^{\prime}\left(t_{n} v_{n}\right)\right\| & =\left\|I_{\lambda}^{\prime}\left(u_{n}\right)\right\| \rightarrow 0 .
\end{aligned}
$$

Desta forma, podemos aplicar o Lema 2.3, para concluir que $\left(y_{n}\right)$ é limitado, digamos $\left\|y_{n}\right\| \leq R$ para algum $R>0$. Portanto, como $y_{n}+B_{1} \subset B_{R+1}$, obtemos

$$
\int_{y_{n}+B_{1}}\left|v_{n}\right|^{2} d x \leq \int_{B_{R+1}}\left|v_{n}\right|^{2} d x
$$

e assim

$$
\int_{B_{R+1}}\left|v_{n}\right|^{2} d x \geq \alpha>0
$$

e, como $v_{n} \rightarrow v$ em $L^{2}\left(B_{R+1}\right)$, segue que

$$
\int_{B_{R+1}}|v|^{2} d x \geq \alpha>0
$$

mostrando que de fato $v \neq 0$.

Por fim, como $v \neq 0$ e $\lambda \neq-\Lambda$, a contrapositiva do Lema 2.4, nos mostra que não podemos ter $t_{n}=\frac{\left\|u_{n}\right\|_{\lambda}}{2 \sqrt{c}} \rightarrow \infty$, logo devemos ter $\left(u_{n}\right)$ limitada. A prova está completa.

Para provar nosso próximo teorema, vamos precisar do seguinte lema, cuja formulação e demonstração podem ser encontradas em Kavian [12] e Lions [14].

Lema 2.5. (Lema de Concentração e Compacidade de Lions) Seja $\left(\rho_{n}\right)$ uma sequência em $L^{1}\left(\mathbb{R}^{N}\right)$, tal que $\rho_{n}>0$ em $\mathbb{R}^{N} e$

$$
\int \rho_{n} d x=\alpha,
$$

onde $\alpha>0$ é um número real fixado. Então existe uma subsequência $\left(\rho_{n_{k}}\right)$ satisfazendo uma das três condições: 
(i) (Anulamento) Para todo $t>0$,

$$
\lim _{k \rightarrow \infty} \sup _{y \in \mathbb{R}^{N}} \int_{y+B_{t}} \rho_{n_{k}} d x=0
$$

(ii) (Dicotomia) Existe $\alpha_{0} \in(0, \alpha)$ tal que, para todo $\varepsilon>0$, existem $k_{0} \geq 1$, uma sequência $\left(y_{n}\right) \subset \mathbb{R}^{N}$, $R>0$ e uma sequência $\left(R_{n}\right) \subset \mathbb{R}^{+}$com $R<R_{1}$ e $R_{n}<R_{n+1} \rightarrow \infty$, de modo que, se:

$$
\bar{\rho}_{n}=\rho_{n} \chi_{\left\{\left|x-y_{n}\right| \leq R\right\}} \quad e \quad \hat{\rho}_{n}=\rho_{n} \chi_{\left\{\left|x-y_{n}\right| \geq R_{n}\right\}}
$$

onde $\chi_{A}$ denota a função característica de $A$, então

$$
\begin{gathered}
\left|\int \bar{\rho}_{k}(x) d x-\alpha_{0}\right| \leq \varepsilon, \quad\left|\int \hat{\rho}_{n}(x) d x-\left(\alpha-\alpha_{0}\right)\right| \leq \varepsilon \quad e \\
\int\left|\rho_{n_{k}}(x)-\left(\bar{\rho}_{k}+\hat{\rho}_{k}\right)(x)\right| d x \leq \varepsilon,
\end{gathered}
$$

para todo $k \geq k_{0} e$

$$
\operatorname{dist}\left(\operatorname{supp}\left(\bar{\rho}_{k}\right), \operatorname{supp}\left(\hat{\rho}_{k}\right)\right) \rightarrow \infty
$$

quando $k \rightarrow \infty$;

(iii) (Compacidade) Existe uma sequência $\left(y_{n}\right) \subset \mathbb{R}^{N}$ tal que, dado $\varepsilon>0$, existe $R>0$ de modo que

$$
\int_{y_{k}+B_{R}} \rho_{n_{k}} d x \geq \alpha-\varepsilon
$$

para todo $k$.

Agora, relembrando a definição da função $h(s)$ em $\left(f_{3}\right)$, vamos considerar o funcional

$$
I_{\lambda}^{\infty}(u)=\frac{1}{2}\|u\|_{\lambda}^{2}-\int H(u) d x, \quad u \in H^{1}\left(\mathbb{R}^{N}\right),
$$

onde $H(s)=\int_{0}^{s} h(t) t d t$. Temos que $I_{\lambda}^{\infty} \in C^{1}\left(\mathbb{R}^{N}, \mathbb{R}\right)$, é tal que

$$
I_{\lambda}^{\infty \prime}(u) v=\int(\nabla u \nabla v+\lambda u v) d x-\int h(u) u v d x, \quad \forall v \in C_{0}^{\infty}\left(\mathbb{R}^{N}\right) .
$$

Definimos a variedade de Nehari dada por

$$
M_{\lambda}^{\infty}=\left\{u \neq 0 \mid I_{\lambda}^{\infty \prime}(u) u=\|u\|_{\lambda}^{2}-\int h(u) u^{2} d x=0\right\} \subset H^{1}\left(\mathbb{R}^{N}\right) .
$$

Também definimos

$$
\begin{aligned}
& 0<m_{\lambda}^{\infty}=\inf _{u \in M_{\lambda}^{\infty}} I_{\lambda}^{\infty}(u), \text { se } M_{\lambda}^{\infty} \neq \varnothing \\
& \text { e } \quad m_{\lambda}^{\infty}=\infty, \quad \text { se } M_{\lambda}^{\infty}=\varnothing \text {. }
\end{aligned}
$$

Estamos prontos para enunciar e provar nosso principal resultado de compacidade. 
Teorema 2.2. Assuma $\left(f_{1}\right)-\left(f_{4}\right)$. Se $0<\lambda<|\Lambda|$, então o funcional $I_{\lambda}$ satisfaz a condição de Cerami $(\mathrm{Ce})_{c}$, para todo $0<c<m_{\lambda}^{\infty}$.

Demonstração. Seja $\left(u_{n}\right)$ uma sequência de Cerami no nível $c \in\left(0, m_{\lambda}^{\infty}\right)$, ou seja

$$
I_{\lambda}\left(u_{n}\right)=\frac{1}{2}\left\|u_{n}\right\|_{\lambda}^{2}-\int F\left(x, u_{n}\right) d x \rightarrow c<m_{\lambda}^{\infty}
$$

e

$$
\left|I_{\lambda}^{\prime}\left(u_{n}\right) \phi\right|=\left|\int\left(\nabla u_{n} \nabla \phi+\lambda u_{n} \phi\right) d x-\int f\left(x, u_{n}\right) u_{n} \phi d x\right| \leq o(1)\|\phi\|_{\lambda} .
$$

Como $\lambda \neq-\Lambda$, então, pela Proposição 2.1, $\left(u_{n}\right)$ é limitado e, sem perda de generalidade, vamos assumir que $\left\|u_{n}\right\|_{\lambda}>0, \quad \forall n \in \mathbb{N}$. Definimos

$$
\rho_{n}:=\left|\nabla u_{n}\right|^{2}+\lambda\left|u_{n}\right|^{2}
$$

logo $\left(\rho_{n}\right)$ é uma sequência em $L^{1}\left(\mathbb{R}^{N}\right)$, pois

$$
\int \rho_{n} d x=\int\left|\nabla u_{n}\right|^{2} d x+\lambda \int\left|u_{n}\right|^{2} d x=\left\|u_{n}\right\|_{\lambda}^{2}<\infty
$$

e passando a uma subsequência se necessário, podemos supor que

$$
\int \rho_{n} d x \rightarrow \alpha \geq 0
$$

quando $n \rightarrow \infty$. Se $\alpha=0$, então $\left\|u_{n}\right\|_{\lambda}^{2} \rightarrow 0$ e por

$$
f(x, s) s^{2} \leq \varepsilon s^{2}+C(\varepsilon, q) s^{q}
$$

e

$$
F(x, s) \leq \frac{1}{2} f(x, s) s^{2},
$$

juntamente com as imersões de Sobolev $H^{1}\left(\mathbb{R}^{N}\right) \hookrightarrow L^{q}\left(\mathbb{R}^{N}\right)$, para $2 \leq q<2^{*}$, temos que

$$
\begin{aligned}
\int F\left(x, u_{n}\right) d x & \leq \frac{1}{2} \int f\left(x, u_{n}\right) u_{n}^{2} d x \\
& \leq \frac{1}{2} \int\left(\varepsilon\left|u_{n}\right|^{2}+C(\varepsilon, q)\left|u_{n}\right|^{q}\right) d x \\
& \leq \frac{1}{2} \varepsilon\left\|u_{n}\right\|_{\lambda}^{2}+C(\varepsilon, q)\left\|u_{n}\right\|_{\lambda}^{q} \rightarrow 0
\end{aligned}
$$

$\operatorname{logo} I_{\lambda}\left(u_{n}\right)=\frac{1}{2}\left\|u_{n}\right\|_{\lambda}^{2}-\int F\left(x, u_{n}\right) d x \rightarrow 0$, o que é uma contradição, pois $I_{\lambda}\left(u_{n}\right) \rightarrow c>0$. Portanto, $\alpha>0$.

Definimos agora

$$
\tilde{\rho}_{n}=\frac{\rho_{n}}{\int \rho_{n} d x}
$$

de modo que $\int \tilde{\rho}_{n} d x=1>0, \forall n \in \mathbb{N}$. Como $\left(\tilde{\rho}_{n}\right)$ satisfaz as hipóteses do Lema 2.5 de Concentração e 
Compacidade de Lions para $\alpha=1$, podemos assumir que $\left(\rho_{n}\right)$ está nas hipóteses do lema, logo

$$
\int \rho_{n} d x=\left\|u_{n}\right\|_{\lambda}^{2} \rightarrow \alpha>0
$$

Vamos considerar cada uma das três possibilidades que pode ocorrer:

1. Anulamento: Suponha que o anulamento ocorra, isto é, para todo $t>0$, temos

$$
\lim _{n \rightarrow \infty} \sup _{y \in \mathbb{R}^{N}} \int_{y+B_{t}}\left(\left|\nabla u_{n}\right|^{2}+\lambda\left|u_{n}\right|^{2}\right) d x=0 .
$$

Neste caso, como vimos na prova da Proposição 2.1, pelo Lema 1.2 de Lions, temos que $u_{n} \rightarrow 0 \mathrm{em}$ $L^{p}\left(\mathbb{R}^{N}\right)$, para qualquer $2<p<2^{*}$. Por $(2.22)$, chegamos em

$$
\int f\left(x, u_{n}\right)\left(u_{n}\right)^{2} d x \leq \varepsilon\left\|u_{n}\right\|_{\lambda}^{2}+C(\varepsilon, q)\left\|u_{n}\right\|_{\lambda}^{q}, \quad \text { para } q \in\left(2,2^{*}\right) .
$$

Tomando $\phi=u_{n}$ em (2.27), seque que

$$
I_{\lambda}^{\prime}\left(u_{n}\right) u_{n}=\left\|u_{n}\right\|_{\lambda}^{2}-\int f\left(x, u_{n}\right) u_{n}^{2} d x \leq o(1)\left\|u_{n}\right\|_{\lambda}, \quad \forall n \in \mathbb{N},
$$

e por (2.28), obtemos

$$
I_{\lambda}^{\prime}\left(u_{n}\right) u_{n} \geq\left\|u_{n}\right\|_{\lambda}^{2}-\varepsilon\left\|u_{n}\right\|_{\lambda}^{2}-C(\varepsilon, q)\left\|u_{n}\right\|_{\lambda}^{q} .
$$

Como $\left(u_{n}\right)$ é limitada em $L^{2}\left(\mathbb{R}^{N}\right),\left\|u_{n}\right\|_{L^{q}\left(\mathbb{R}^{N}\right)} \rightarrow 0$ e $\left\|u_{n}\right\|_{\lambda}^{2} \rightarrow \alpha>0$, então para todo $\varepsilon>0$ pequeno, temos

$$
o(1)+\frac{\alpha}{2} \leq I_{\lambda}^{\prime}\left(u_{n}\right) u_{n} \leq o(1),
$$

o que é uma contradição, logo não ocorre o anulamento.

2. Dicotomia: Suponha que a dicotomia ocorra. Então existe $\alpha_{0}$ tal que $0<\alpha_{0}<\alpha$ onde, para $\varepsilon>0$ dado, existem $R>0$ e sequências

$$
\left(y_{n}\right) \subset \mathbb{R}^{N}, \quad\left(R_{n}\right) \subset \mathbb{R}^{+},
$$

$\operatorname{com} R<R_{1}, R_{n}<R_{n+1} \rightarrow \infty$, tal que

$$
\begin{gathered}
\alpha_{0}-\varepsilon \leq \int_{\left|x-y_{n}\right| \leq R / 2}\left(\left|\nabla u_{n}\right|^{2}+\lambda\left|u_{n}\right|^{2}\right) d x \leq \alpha_{0}+\varepsilon, \\
\alpha-\alpha_{0}-\varepsilon \leq \int_{\left|x-y_{n}\right| \geq 3 R_{n}}\left(\left|\nabla u_{n}\right|^{2}+\lambda\left|u_{n}\right|^{2}\right) d x \leq\left(\alpha-\alpha_{0}\right)+\varepsilon,
\end{gathered}
$$

e, em particular, sabendo que

$$
\alpha+o(1)=\int\left(\left|\nabla u_{n}\right|^{2}+\lambda\left|u_{n}\right|^{2}\right) d x
$$


teremos

$$
\begin{aligned}
\int_{R / 2 \leq\left|x-y_{n}\right| \leq 3 R_{n}}\left(\left|\nabla u_{n}\right|^{2}+\lambda\left|u_{n}\right|^{2}\right) d x & =\alpha+o(1)-\int_{\left|x-y_{n}\right| \geq 3 R_{n}}\left(\left|\nabla u_{n}\right|^{2}+\lambda\left|u_{n}\right|^{2}\right) d x \\
& -\int_{\left|x-y_{n}\right| \leq R / 2}\left(\left|\nabla u_{n}\right|^{2}+\lambda\left|u_{n}\right|^{2}\right) d x \\
& \leq \alpha+o(1)-\alpha_{0}+\varepsilon-\alpha+\alpha_{0}+\varepsilon \\
& =2 \varepsilon+o(1),
\end{aligned}
$$

isto é,

$$
\int_{R / 2 \leq\left|x-y_{n}\right| \leq 3 R_{n}}\left(\left|\nabla u_{n}\right|^{2}+\lambda\left|u_{n}\right|^{2}\right) d x \leq 2 \varepsilon+o(1) .
$$

Considere $\zeta \in C_{0}^{\infty}\left(\mathbb{R}^{N}\right)$, tal que $0 \leq \zeta(x) \leq 1 \mathrm{e}$

$$
\zeta(x)= \begin{cases}1, & \text { se }|x| \leq 1 \\ 0, & \text { se }|x| \geq 2\end{cases}
$$

e considere também $\varphi=1-\zeta$, logo $0 \leq \varphi \leq 1 \mathrm{e}$

$$
\varphi(x)= \begin{cases}0, & \text { se }|x| \leq 1 \\ 1, & \text { se }|x| \geq 2\end{cases}
$$

Definimos as sequências

$$
\zeta_{n}(x)=\zeta\left(\frac{x-y_{n}}{R}\right) \text { e } \varphi_{n}(x)=\varphi\left(\frac{x-y_{n}}{R_{n}}\right), \text { para } x \in \mathbb{R}^{N},
$$

e defina também

$$
u_{n}^{1}(x):=\zeta_{n}(x) u_{n}(x) \quad \text { e } \quad u_{n}^{2}(x):=\varphi_{n}(x) u_{n}(x) .
$$

Logo, temos que, para todo $n \in \mathbb{N}$,

$$
u_{n}^{1}(x)= \begin{cases}u_{n}(x), & \text { se }\left|x-y_{n}\right| \leq R \\ 0, & \text { se }\left|x-y_{n}\right| \geq 2 R\end{cases}
$$

$$
u_{n}^{2}(x)= \begin{cases}0, & \text { se }\left|x-y_{n}\right| \leq R_{n} \\ u_{n}(x), & \text { se }\left|x-y_{n}\right| \geq 2 R_{n}\end{cases}
$$

Afirmamos que

$$
I_{\lambda}\left(u_{n}\right) \geq I_{\lambda}\left(u_{n}^{1}\right)+I_{\lambda}\left(u_{n}^{2}\right)-C \varepsilon,
$$

para $C>0$. De fato, denotando por

$$
\rho_{n}^{i}=\left|\nabla u_{n}^{i}\right|^{2}+\lambda\left(u_{n}^{i}\right)^{2}, \quad i=1,2,
$$

temos

$$
\begin{gathered}
\left|I_{\lambda}\left(u_{n}\right)-I_{\lambda}\left(u_{n}^{1}\right)-I_{\lambda}\left(u_{n}^{2}\right)\right|= \\
\left|\frac{1}{2} \int \rho_{n} d x-\int F\left(x, u_{n}\right) d x-\frac{1}{2} \int \rho_{n}^{1} d x+\int F\left(x, u_{n}^{1}\right) d x-\frac{1}{2} \int \rho_{n}^{2} d x+\int F\left(x, u_{n}^{2}\right) d x\right| .
\end{gathered}
$$


Definindo $A_{n}:=\left\{x \in \mathbb{R}^{N}\left|\frac{R}{2} \leq\right| x-y_{n} \mid \leq 3 R_{n}\right\}$, temos:

$$
\begin{aligned}
\int \rho_{n} d x-\int \rho_{n}^{1} d x-\int \rho_{n}^{2} d x & =\int_{\left|x-y_{n}\right| \leq R / 2} \rho_{n} d x+\int_{A_{n}} \rho_{n} d x+\int_{\left|x-y_{n}\right| \geq 3 R_{n}} \rho_{n} d x \\
& -\int_{\left|x-y_{n}\right| \leq R / 2} \rho_{n}^{1} d x-\int_{A_{n}} \rho_{n}^{1} d x-\int_{\left|x-y_{n}\right| \geq 3 R_{n}} \rho_{n}^{1} d x \\
& -\int_{\left|x-y_{n}\right| \leq R / 2} \rho_{n}^{2} d x-\int_{A_{n}} \rho_{n}^{2} d x-\int_{\left|x-y_{n}\right| \geq 3 R_{n}} \rho_{n}^{2} d x
\end{aligned}
$$

e pela definição de $u_{n}^{i}$ e de $\rho_{n}$, temos que

$$
\int \rho_{n} d x-\int \rho_{n}^{1} d x-\int \rho_{n}^{2} d x=\int_{A_{n}} \rho_{n} d x-\int_{A_{n}} \rho_{n}^{1} d x-\int_{A_{n}} \rho_{n}^{2} d x
$$

e, analogamente,

$$
-\int F\left(x, u_{n}\right) d x+\int F\left(x, u_{n}^{1}\right) d x+\int F\left(x, u_{n}^{2}\right) d x=-\int_{A_{n}} F\left(x, u_{n}\right) d x+\int_{A_{n}} F\left(x, u_{n}^{1}\right) d x+\int_{A_{n}} F\left(x, u_{n}^{2}\right) d x .
$$

Assim, temos que

$$
\begin{aligned}
\left|I_{\lambda}\left(u_{n}\right)-I_{\lambda}\left(u_{n}^{1}\right)-I_{\lambda}\left(u_{n}^{2}\right)\right| & =\mid \frac{1}{2} \int_{A_{n}} \rho_{n} d x-\int_{A_{n}} F\left(x, u_{n}\right) d x-\frac{1}{2} \int_{A_{n}} \rho_{n}^{1} d x \\
& +\int_{A_{n}} F\left(x, u_{n}^{1}\right) d x-\frac{1}{2} \int_{A_{n}} \rho_{n}^{2} d x+\int_{A_{n}} F\left(x, u_{n}^{2}\right) d x \mid .
\end{aligned}
$$

Temos $\int_{A_{n}} \rho_{n} d x \leq 2 \varepsilon+o(1)$, por (2.31). Vamos agora estimar os outros termos da expressão dada em (2.33), vamos estimar primeiramente $\int_{A_{n}}\left|\nabla u_{n}^{1}\right|^{2} d x$. Como

$$
\nabla u_{n}^{1}=\frac{1}{R}\left(\nabla \zeta_{n}\right) u_{n}+\zeta_{n}\left(\nabla u_{n}\right)
$$

temos

$$
\begin{aligned}
\int_{A_{n}}\left|\nabla u_{n}^{1}\right|^{2} d x & =\int_{A_{n}}\left|\frac{1}{R}\left(\nabla \zeta_{n}\right) u_{n}+\zeta_{n}\left(\nabla u_{n}\right)\right|^{2} d x \\
& \leq \frac{2}{R^{2}} \int_{A_{n}}\left|\nabla \zeta_{n}\right|^{2}\left(u_{n}\right)^{2} d x+2 \int_{A_{n}} \zeta_{n}\left|\nabla u_{n}\right|^{2} d x \\
& \leq \frac{2}{R^{2}} \int_{A_{n}}\left|\nabla \zeta_{n}\right|^{2}\left(u_{n}\right)^{2} d x+4 \varepsilon
\end{aligned}
$$

Agora, utilizando a desigualdade de Hölder com os expoentes conjugados $\frac{1}{N / 2}+\frac{1}{N /(N-2)}=1$, teremos que

$$
\int_{A_{n}}\left|\nabla \zeta_{n}\right|^{2}\left(u_{n}\right)^{2} d x \leq\left(\int_{A_{n}}\left|\nabla \zeta_{n}\right|^{N} d x\right)^{2 / N}\left(\int_{A_{n}}\left|u_{n}\right|^{2^{*}} d x\right)^{2 / 2^{*}}
$$


e como $\left\|\nabla \zeta_{n}\right\|_{L^{N}\left(\mathbb{R}^{N}\right)}=\|\nabla \zeta\|_{L^{N}\left(\mathbb{R}^{N}\right)}$ e $H^{1}\left(A_{n}\right) \hookrightarrow L^{2^{*}}\left(A_{n}\right)$, teremos

$$
\int_{A_{n}}\left|\nabla \zeta_{n}\right|^{2}\left(u_{n}\right)^{2} d x \leq\|\nabla \zeta\|_{L^{N}\left(\mathbb{R}^{N}\right)}^{2}\left(\int_{A_{n}}\left(\left|\nabla u_{n}\right|^{2}+\lambda\left(u_{n}\right)^{2}\right) d x\right) \leq C \varepsilon .
$$

Portanto

$$
\int_{A_{n}}\left|\nabla u_{n}^{1}\right|^{2} d x \leq C \varepsilon
$$

Agora com o fato de que $\left|u_{n}^{1}\right| \leq\left|u_{n}\right|$ e usando (2.31), obtemos

$$
\int_{A_{n}} \rho_{n}^{1} d x \leq C \varepsilon+\int_{A_{n}} \lambda\left(u_{n}\right)^{2} d x \leq C \varepsilon
$$

e, analogamente,

$$
\int_{A_{n}} \rho_{n}^{2} d x \leq C \varepsilon .
$$

Como $0 \leq F(x, s) \leq C s^{2}$ e $\left|u_{n}^{1}\right| \leq\left|u_{n}\right|$, segue que

$$
\int_{A_{n}} F\left(x, u_{n}^{1}\right) d x \leq C \int_{A_{n}}\left(u_{n}^{1}\right)^{2} d x \leq \int_{A_{n}}\left(u_{n}\right)^{2} d x \leq C \varepsilon,
$$

e, portanto,

$$
\int_{A_{n}} F\left(x, u_{n}^{1}\right) d x \leq C \varepsilon \quad \text { e } \quad \int_{A_{n}} F\left(x, u_{n}^{2}\right) d x \leq C \varepsilon .
$$

Assim, segue de (2.33) - (2.37), que

$$
\left|I_{\lambda}\left(u_{n}\right)-I_{\lambda}\left(u_{n}^{1}\right)-I_{\lambda}\left(u_{n}^{2}\right)\right| \leq C \varepsilon,
$$

mostrando (2.32).

Afirmamos agora que

$$
\left|\left\|u_{n}^{1}\right\|_{\lambda}^{2}-\int f\left(x, u_{n}^{1}\right)\left(u_{n}^{1}\right)^{2} d x\right| \leq C \varepsilon
$$

para alguma constante $C>0$. De fato, da definição de $u_{n}^{1}$, temos

$$
\begin{array}{r}
\left|I_{\lambda}^{\prime}\left(u_{n}\right)\left(u_{n}^{1}\right)-\left[\left\|u_{n}^{1}\right\|_{\lambda}^{2}-\int f\left(x, u_{n}^{1}\right)\left(u_{n}^{1}\right)^{2} d x\right]\right| \\
=\mid \int_{A_{n}}\left(\nabla u_{n} \nabla u_{n}^{1}+\lambda u_{n} u_{n}^{1}\right) d x-\int_{A_{n}} f\left(x, u_{n}\right) u_{n} u_{n}^{1} d x \\
-\int_{A_{n}}\left(\left|\nabla u_{n}^{1}\right|^{2}+\lambda\left(u_{n}^{1}\right)^{2}\right) d x+\int_{A_{n}} f\left(x, u_{n}^{1}\right)\left(u_{n}^{1}\right)^{2} d x \mid .
\end{array}
$$

Usando o fato de que $f$ é não-decrescente na segunda coordenada, $\left|u_{n}^{1}\right| \leq\left|u_{n}\right|$ e (2.22), obtemos

$$
\begin{aligned}
f\left(x, u_{n}^{1}\right)\left(u_{n}^{1}\right)^{2} & \leq f\left(x, u_{n}\right)\left|u_{n} u_{n}^{1}\right| \\
& \leq f\left(x, u_{n}\right)\left(u_{n}\right)^{2} \\
& \leq \varepsilon\left|u_{n}\right|^{2}+C(\varepsilon, q)\left|u_{n}\right|^{q} .
\end{aligned}
$$


Agora da imersão $H^{1}\left(A_{n}\right) \hookrightarrow L^{q}\left(A_{n}\right)$, temos

$$
\begin{aligned}
\left|\int_{A_{n}} f\left(x, u_{n}\right) u_{n} u_{n}^{1} d x\right| & \leq \int_{A_{n}}\left(\varepsilon\left|u_{n}\right|^{2}+C(\varepsilon, q)\left|u_{n}\right|^{q}\right) d x \\
& \leq \varepsilon\left\|u_{n}\right\|_{L^{2}\left(A_{n}\right)}^{2}+C(\varepsilon, q)\left[\int_{A_{n}}\left(\left|\nabla u_{n}\right|^{2}+\lambda\left(u_{n}\right)^{2}\right) d x\right]^{q / 2} \\
& \leq C \varepsilon
\end{aligned}
$$

e de forma análoga, mostra-se que

$$
\int_{A_{n}} f\left(x, u_{n}^{1}\right)\left(u_{n}^{1}\right)^{2} d x \leq C \varepsilon
$$

Usando a desigualdade de Hölder, juntamente com (2.34), segue que

$$
\int_{A_{n}}\left(\nabla u_{n} \nabla u_{n}^{1}+\lambda u_{n} u_{n}^{1}\right) d x \leq\left(\int_{A_{n}} \rho_{n} d x\right)^{1 / 2}\left(\int_{A_{n}} \rho_{n}^{1} d x\right)^{1 / 2} \leq C \varepsilon .
$$

Logo, por (2.39) - (2.42), obtemos

$$
\left|I_{\lambda}^{\prime}\left(u_{n}\right)\left(u_{n}^{1}\right)-\left(\left\|u_{n}^{1}\right\|_{\lambda}^{2}-\int f\left(x, u_{n}^{1}\right)\left(u_{n}^{1}\right)^{2} d x\right)\right| \leq C \varepsilon
$$

ou seja,

$$
\left|\left\|u_{n}^{1}\right\|_{\lambda}^{2}-\int f\left(x, u_{n}^{1}\right)\left(u_{n}^{1}\right)^{2} d x\right| \leq\left|I_{\lambda}^{\prime}\left(u_{n}\right)\left(u_{n}^{1}\right)\right|+C \varepsilon
$$

Usando que

$$
\left|I_{\lambda}^{\prime}\left(u_{n}\right) \phi\right|=\left|\int\left(\nabla u_{n} \nabla \phi+\lambda u_{n} \phi\right) d x-\int f\left(x, u_{n}\right) u_{n} \phi d x\right| \leq \varepsilon\|\phi\|_{\lambda},
$$

obtemos

$$
\left|\left\|u_{n}^{1}\right\|_{\lambda}^{2}-\int f\left(x, u_{n}^{1}\right)\left(u_{n}^{1}\right)^{2} d x\right| \leq C \varepsilon
$$

para algum $C>0$ e $n$ suficientemente grande.

De forma inteiramente análoga, mostra-se que

$$
\left|\left\|u_{n}^{2}\right\|_{\lambda}^{2}-\int f\left(x, u_{n}^{2}\right)\left(u_{n}^{2}\right)^{2} d x\right| \leq C \varepsilon
$$

Para mostrar que a dicotomia não ocorre, consideraremos dois casos:

Caso 1: $\left(y_{n}\right) \subset \mathbb{R}^{N}$ é limitada.

Como $\left(y_{n}\right)$ é limitada, temos que os centros das bolas $B_{R_{n}}\left(y_{n}\right)$ não convergem para o infinito, e como $R_{n} \rightarrow \infty$, temos que as bolas crescem de acordo com $n$, a partir de um $n_{0}$ suficientemente grande. Desde que $u_{n}^{2}(x)=0$ se $\left|x-y_{n}\right| \leq R_{n}$, tem-se

$$
\operatorname{supp}\left(u_{n}^{2}\right) \subset \mathbb{R}^{N} \backslash B_{R_{n}}\left(y_{n}\right)
$$


Do fato $\lim _{|x| \rightarrow \infty} f(x, s)=h(s)$, temos que dado $\varepsilon>0$, obtemos

$$
\left|f\left(x, u_{n}^{2}\right)-h\left(u_{n}^{2}\right)\right| \leq \varepsilon^{1 / 2},
$$

para $|x|>r>0$ suficientemente grande.

Como $\left(u_{n}^{2}\right)^{2}$ é integrável, temos, para $r$ grande, que

$$
\int_{|x|>r}\left(u_{n}^{2}\right)^{2} d x \leq \varepsilon^{1 / 2} .
$$

Para $n$ grande, ainda temos que

$$
\operatorname{supp}\left(u_{n}^{2}\right) \subset \mathbb{R}^{N} \backslash B_{R_{n}}\left(y_{n}\right) \subset \mathbb{R}^{N} \backslash B_{r}(0) .
$$

Portanto,

$$
\begin{aligned}
\left|\int\left[f\left(x, u_{n}^{2}\right) u_{n}^{2}-h\left(u_{n}^{2}\right) u_{n}^{2}\right] d x\right| & =\left|\int_{\operatorname{supp}\left(u_{n}^{2}\right)}\left[f\left(x, u_{n}^{2}\right)-h\left(u_{n}^{2}\right)\right] u_{n}^{2} d x\right| \\
& \leq \varepsilon^{1 / 2} \int_{\operatorname{supp}\left(u_{n}^{2}\right)}\left(u_{n}^{2}\right)^{2} d x \\
& \leq \varepsilon .
\end{aligned}
$$

De (2.45) e (2.46), segue que

$$
\begin{aligned}
\left|I_{\lambda}^{\infty \prime}\left(u_{n}^{2}\right)\left(u_{n}^{2}\right)\right| & =\left|\left\|u_{n}^{2}\right\|_{\lambda}^{2}-\int h\left(u_{n}^{2}\right)\left(u_{n}^{2}\right)^{2} d x\right| \\
& \leq\left|\left\|u_{n}^{2}\right\|_{\lambda}^{2}-\int f\left(x, u_{n}^{2}\right)\left(u_{n}^{2}\right)^{2} d x\right|+\left|\int\left[f\left(x, u_{n}^{2}\right) u_{n}^{2}-h\left(u_{n}^{2}\right) u_{n}^{2}\right] d x\right| \\
& \leq C \varepsilon .
\end{aligned}
$$

Dado $\varepsilon>0$, temos por $\lim _{|x| \rightarrow \infty} f(x, s)=h(s)$ que

$$
|f(x, s)-h(s)| \leq \varepsilon^{1 / 2},
$$

para $s$ grande. Logo

$$
\begin{aligned}
\left|\int\left[F\left(x, u_{n}^{2}\right)-H\left(u_{n}^{2}\right)\right] d x\right| & \leq \int_{\operatorname{supp}\left(u_{n}^{2}\right)} \int_{0}^{u_{n}^{2}}|f(x, s)-h(s)| s d s d x \\
& \leq \int_{\operatorname{supp}\left(u_{n}^{2}\right)} \int_{0}^{u_{n}^{2}} \varepsilon^{1 / 2} s d s d x \\
& =\frac{1}{2} \varepsilon^{1 / 2} \int_{\operatorname{supp}\left(u_{n}^{2}\right)}\left(u_{n}^{2}\right)^{2} d x \leq \varepsilon .
\end{aligned}
$$

Portanto,

$$
\left|I_{\lambda}\left(u_{n}^{2}\right)-I_{\lambda}^{\infty}\left(u_{n}^{2}\right)\right|=\left|-\int F\left(x, u_{n}^{2}\right) d x+\int H\left(u_{n}^{2}\right) d x\right| \leq \varepsilon
$$

e assim,

$$
I_{\lambda}\left(u_{n}^{2}\right)=I_{\lambda}^{\infty}\left(u_{n}^{2}\right)+o(1)
$$


Mostraremos que, se a dicotomia ocorre, então $M_{\lambda}^{\infty} \neq \varnothing$. Para isso, vamos definir

$$
w_{n}^{2}:=u_{n}^{2}(\sigma x), \quad 0 \neq \sigma \in \mathbb{R}
$$

Por mudança de variáveis, temos

$$
\begin{aligned}
I_{\lambda}^{\infty \prime}\left(w_{n}^{2}\right)\left(w_{n}^{2}\right) & =\int\left(\left|\nabla w_{n}^{2}\right|^{2}+\lambda\left(w_{n}^{2}\right)^{2}\right) d x-\int h\left(w_{n}^{2}\right)\left(w_{n}^{2}\right)^{2} d x \\
& =\int\left(\sigma^{2}\left|\nabla u_{n}^{2}(\sigma x)\right|^{2}+\lambda\left(u_{n}^{2}(\sigma x)\right)^{2}\right) d x-\int h\left(u_{n}^{2}(\sigma x)\right)\left(u_{n}^{2}(\sigma x)\right)^{2} d x \\
& =\int\left(\sigma^{2-N}\left|\nabla u_{n}^{2}(x)\right|^{2}+\sigma^{-N} \lambda\left(u_{n}^{2}(x)\right)^{2}\right) d x-\sigma^{-N} \int h\left(u_{n}^{2}(x)\right)\left(u_{n}^{2}(x)\right)^{2} d x \\
& =\sigma^{-N}\left[\left(\sigma^{2}-1\right) \int\left|\nabla u_{n}^{2}\right|^{2} d x+\int\left(\left|\nabla u_{n}^{2}\right|^{2}+\lambda\left(u_{n}^{2}\right)^{2}\right) d x-\int h\left(u_{n}^{2}\right)\left(u_{n}^{2}\right)^{2} d x\right] .
\end{aligned}
$$

Por (2.47), denotamos

$$
\varepsilon_{n}=\int\left(\left|\nabla u_{n}^{2}\right|^{2}+\lambda\left(u_{n}^{2}\right)^{2}\right) d x-\int h\left(u_{n}^{2}\right)\left(u_{n}^{2}\right)^{2} d x
$$

$\operatorname{com} \varepsilon_{n} \rightarrow 0$. Portanto

$$
I_{\lambda}^{\infty \prime}\left(w_{n}^{2}\right)\left(w_{n}^{2}\right)=\sigma^{-N}\left[\left(\sigma^{2}-1\right) \int\left|\nabla u_{n}^{2}\right|^{2} d x+\varepsilon_{n}\right]
$$

Para que $M_{\lambda}^{\infty}$ seja não-vazio, é suficiente que $\int\left|\nabla u_{n}^{2}\right|^{2} d x:=a_{n}>0$, para $n$ grande. De fato, se $a_{n}>0$, para $n$ grande, então tomamos

$$
\sigma_{n}=\left(1-\frac{\varepsilon_{n}}{a_{n}}\right)^{1 / 2}
$$

Se $\varepsilon_{n} \leq 0$, então $1-\frac{\varepsilon_{n}}{a_{n}}>0$ e se $\varepsilon_{n}>0$, como $a_{n}$ é limitada, já que $\left(u_{n}^{2}\right)$ também é em $H^{1}\left(\mathbb{R}^{N}\right)$, é possível tomar $\varepsilon_{n}$ pequeno, tal que $1-\frac{\varepsilon_{n}}{a_{n}}>0$. Logo,

$$
\left(\sigma_{n}^{2}-1\right) \int\left|\nabla u_{n}^{2}\right|^{2} d x+\varepsilon_{n}=0
$$

isto é,

$$
I_{\lambda}^{\infty \prime}\left(w_{n}^{2}\right)\left(w_{n}^{2}\right)=0
$$

e $w_{n}^{2} \in M_{\lambda}^{\infty}$. Logo, se a dicotomia ocorrer, teríamos que $M_{\lambda}^{\infty} \neq \varnothing$.

Precisamos então provar que

$$
\int\left|\nabla u_{n}^{2}\right|^{2} d x \geq C>0
$$

De fato, suponhamos que exista uma subsequência de $\left(u_{n}^{2}\right)$, também denotada por $\left(u_{n}^{2}\right)$, tal que

$$
\int\left|\nabla u_{n}^{2}\right|^{2} d x \rightarrow 0
$$

Como estamos assumindo a dicotomia, temos

$$
\left|\int \hat{\rho}_{n} d x-\left(\alpha-\alpha_{0}\right)\right|=\left|\int_{\left|x-y_{n}\right|>R_{n}}\left(\left|\nabla u_{n}\right|^{2}+\lambda\left(u_{n}\right)^{2}\right) d x-\left(\alpha-\alpha_{0}\right)\right| \leq \varepsilon
$$


Da definição de $u_{n}^{2}$, temos

$$
\int_{\left|x-y_{n}\right| \geq R_{n}}\left[\left(u_{n}^{2}\right)^{2}-\left(u_{n}\right)^{2}\right] d x=\int_{R_{n} \leq\left|x-y_{n}\right| \leq 2 R_{n}}\left(u_{n}^{2}\right)^{2} d x
$$

$\log O$

|| $\int_{\left|x-y_{n}\right| \geq 2 R_{n}}\left|\nabla u_{n}\right|^{2} d x+\int_{\left|x-y_{n}\right| \geq R_{n}} \lambda\left(u_{n}^{2}\right)^{2} d x-\left(\alpha-\alpha_{0}\right)|-| \int_{\left|x-y_{n}\right| \geq R_{n}}\left(\left|\nabla u_{n}\right|^{2}+\lambda u_{n}^{2}\right) d x-\left(\alpha-\alpha_{0}\right)|| \leq \varepsilon$, para $n$ grande, assim

$$
\int_{\left|x-y_{n}\right| \geq 2 R_{n}}\left|\nabla u_{n}\right|^{2} d x+\int_{\left|x-y_{n}\right| \geq R_{n}} \lambda\left(u_{n}^{2}\right)^{2} d x \geq\left(\alpha-\alpha_{0}\right)-\varepsilon
$$

Como $\varphi_{n}(x)=1$, se $\left|x-y_{n}\right| \geq 2 R_{n}$, obtemos

$$
\begin{aligned}
\int_{\left|x-y_{n}\right| \geq R_{n}}\left|\nabla u_{n}^{2}\right|^{2} d x & =\int_{\left|x-y_{n}\right| \geq R_{n}}\left|\frac{1}{R_{n}} u_{n}\left(\nabla \varphi_{n}\right)+\varphi_{n}\left(\nabla u_{n}\right)\right|^{2} d x \\
& \geq \int_{\left|x-y_{n}\right| \geq 2 R_{n}}\left|\frac{1}{R_{n}} u_{n}\left(\nabla \varphi_{n}\right)+\varphi_{n}\left(\nabla u_{n}\right)\right|^{2} d x \\
& =\int_{\left|x-y_{n}\right| \geq 2 R_{n}}\left|\nabla u_{n}\right|^{2} d x
\end{aligned}
$$

Logo, por (2.52), e o fato que $u_{n}^{2}(x)=0$ se $\left|x-y_{n}\right| \leq R_{n}$, tem-se

$$
\int\left(\left|\nabla u_{n}^{2}\right|^{2}+\lambda\left(u_{n}^{2}\right)^{2}\right) d x \geq \int_{\left|x-y_{n}\right| \geq 2 R_{n}}\left|\nabla u_{n}\right|^{2} d x+\int_{\left|x-y_{n}\right| \geq R_{n}} \lambda\left(u_{n}^{2}\right)^{2} d x \geq\left(\alpha-\alpha_{0}\right)-\varepsilon .
$$

Agora por $f(x, s) s^{2} \leq \varepsilon s^{2}+C(\varepsilon, q) s^{q}$ e $(2.45)$, temos que

$$
\begin{aligned}
\int\left(\left|\nabla u_{n}^{2}\right|^{2}+\lambda\left(u_{n}^{2}\right)^{2}\right) d x & \leq \int f\left(x, u_{n}^{2}\right)\left(u_{n}^{2}\right)^{2} d x+C \varepsilon \\
& \leq \int\left(\varepsilon\left(u_{n}^{2}\right)^{2}+C\left(\varepsilon, 2^{*}\right)\left(u_{n}^{2}\right)^{2^{*}}\right) d x+C \varepsilon \\
& =\varepsilon \int\left(u_{n}^{2}\right)^{2} d x+C\left(\varepsilon, 2^{*}\right) \int\left(u_{n}^{2}\right)^{2^{*}} d x+C \varepsilon
\end{aligned}
$$

e usando a imersão $D^{1,2}\left(\mathbb{R}^{N}\right) \hookrightarrow L^{2^{*}}\left(\mathbb{R}^{N}\right)$, obtemos

$$
\int\left(\left|\nabla u_{n}^{2}\right|^{2}+\lambda\left(u_{n}^{2}\right)^{2}\right) d x \leq \varepsilon \int\left(u_{n}^{2}\right)^{2} d x+C_{1}\left(\varepsilon, 2^{*}\right) \int\left|\nabla u_{n}^{2}\right|^{2} d x+C \varepsilon .
$$

Logo de (2.53) e da desigualdade acima, temos

$$
\left(\alpha-\alpha_{0}\right)-\varepsilon \leq \varepsilon \int\left(u_{n}^{2}\right)^{2} d x+C_{1}\left(\varepsilon, 2^{*}\right) \int\left|\nabla u_{n}^{2}\right|^{2} d x+C \varepsilon
$$

o que é um absurdo, pois de $(2.51)$ e do fato de que $\left(u_{n}^{2}\right)$ é limitada em $L^{2}\left(\mathbb{R}^{N}\right)$, podemos tomar $\varepsilon \rightarrow 0$ e $n \rightarrow \infty$, para concluir que

$$
0<\left(\alpha-\alpha_{0}\right) \leq 0
$$


Assim, temos de fato que

$$
\int\left|\nabla u_{n}^{2}\right|^{2} d x \geq C>0
$$

$\operatorname{logo} M_{\lambda}^{\infty} \neq \varnothing$, isso se a dicotomia ocorrer.

Vamos verificar agora que de fato a dicotomia não pode acontecer. Fazendo uma mudança de variáveis, temos

$$
\begin{aligned}
I_{\lambda}^{\infty}\left(w_{n}^{2}\right) & =\frac{1}{2} \int\left(\left|\nabla w_{n}^{2}\right|^{2}+\lambda\left(w_{n}^{2}\right)^{2}\right) d x-\int H\left(w_{n}^{2}\right) d x \\
& =\frac{1}{2} \int\left(\sigma_{n}^{2}\left|\nabla u_{n}^{2}\left(\sigma_{n} x\right)\right|^{2}+\lambda\left(u_{n}^{2}\left(\sigma_{n} x\right)\right)^{2}\right) d x-\int H\left(u_{n}^{2}\left(\sigma_{n} x\right)\right) d x \\
& =\frac{1}{2} \sigma_{n}^{-N} \int\left(\sigma_{n}^{2}\left|\nabla u_{n}^{2}(x)\right|^{2}+\lambda\left(u_{n}^{2}(x)\right)^{2}\right) d x-\sigma_{n}^{-N} \int H\left(u_{n}^{2}(x)\right) d x \\
& =\frac{1}{2} \sigma_{n}^{-N}\left(\sigma_{n}^{2}-1\right) \int\left|\nabla u_{n}^{2}\right|^{2} d x+\sigma_{n}^{-N}\left[\frac{1}{2} \int\left(\left|\nabla u_{n}^{2}\right|^{2}+\lambda\left(u_{n}^{2}\right)^{2}\right) d x-\int H\left(u_{n}^{2}\right) d x\right] \\
& =\frac{1}{2} \sigma_{n}^{-N}\left(\sigma_{n}^{2}-1\right) \int\left|\nabla u_{n}^{2}\right|^{2} d x+\sigma_{n}^{-N} I_{\lambda}^{\infty}\left(u_{n}^{2}\right) \\
& =\frac{1}{2} \sigma_{n}^{-N}\left(\sigma_{n}^{2}-1\right) \int\left|\nabla u_{n}^{2}\right|^{2} d x+\left(\sigma_{n}^{-N}-1\right) I_{\lambda}^{\infty}\left(u_{n}^{2}\right)+I_{\lambda}^{\infty}\left(u_{n}^{2}\right),
\end{aligned}
$$

isto é,

$$
I_{\lambda}^{\infty}\left(w_{n}^{2}\right)=I_{\lambda}^{\infty}\left(u_{n}^{2}\right)+\frac{1}{2} \sigma_{n}^{-N}\left(\sigma_{n}^{2}-1\right) \int\left|\nabla u_{n}^{2}\right|^{2} d x+\left(\sigma_{n}^{-N}-1\right) I_{\lambda}^{\infty}\left(u_{n}^{2}\right)
$$

Afirmamos que $I_{\lambda}^{\infty}\left(u_{n}^{2}\right)$ é limitado. De fato, usando $(2.48)$ e $F\left(x, u_{n}^{2}\right) \leq C\left(u_{n}^{2}\right)$, obtemos

$$
\begin{aligned}
\left|I_{\lambda}^{\infty}\left(u_{n}^{2}\right)\right| & =\left|\frac{1}{2}\left\|u_{n}^{2}\right\|_{\lambda}^{2}-\int H\left(u_{n}^{2}\right) d x\right| \\
& \leq \frac{1}{2}\left\|u_{n}^{2}\right\|_{\lambda}^{2}+\left|\int\left(F\left(x, u_{n}^{2}\right)-H\left(u_{n}^{2}\right)\right) d x\right|+\left|\int F\left(x, u_{n}^{2}\right) d x\right| \\
& \leq \frac{1}{2}\left\|u_{n}^{2}\right\|_{\lambda}^{2}+C\left\|u_{n}^{2}\right\|_{L^{2}\left(\mathbb{R}^{N}\right)}^{2}+\varepsilon
\end{aligned}
$$

e como $\left(u_{n}^{2}\right)$ é limitado, então está provada a afirmação.

Agora, usando (2.49) e (2.54), tem-se que

$$
\begin{aligned}
I_{\lambda}\left(u_{n}^{2}\right) & \geq I_{\lambda}^{\infty}\left(u_{n}^{2}\right)-\varepsilon \\
& =I_{\lambda}^{\infty}\left(w_{n}^{2}\right)-\frac{1}{2} \sigma_{n}^{-N}\left(\sigma_{n}^{2}-1\right) \int\left|\nabla u_{n}^{2}\right|^{2} d x-\left(\sigma_{n}^{-N}-1\right) I_{\lambda}^{\infty}\left(u_{n}^{2}\right)-\varepsilon
\end{aligned}
$$

Fazendo $\left(\sigma_{n}^{2}-1\right) \int\left|\nabla u_{n}^{2}\right|^{2} d x=-\varepsilon_{n}$, por $(2.50)$, onde $\sigma_{n}=\left(1-\frac{\varepsilon_{n}}{a_{n}}\right)^{1 / 2}$, temos, para $\varepsilon=\left|\varepsilon_{n}\right|$, que

$$
I_{\lambda}\left(u_{n}^{2}\right) \geq I_{\lambda}^{\infty}\left(w_{n}^{2}\right)+\frac{1}{2} \sigma_{n}^{-N} \varepsilon_{n}-\left(\sigma_{n}^{-N}-1\right) I_{\lambda}^{\infty}\left(u_{n}^{2}\right)-\left|\varepsilon_{n}\right|
$$

Desde que $\sigma_{n} \rightarrow 1$ quando $\varepsilon_{n} \rightarrow 0$ e $I_{\lambda}^{\infty}\left(u_{n}^{2}\right)$ é limitado, temos

$$
I_{\lambda}\left(u_{n}^{2}\right) \geq I_{\lambda}^{\infty}\left(w_{n}^{2}\right)-\overline{\varepsilon_{n}}
$$

onde $\overline{\varepsilon_{n}}>0$ e $\overline{\varepsilon_{n}} \rightarrow 0$, ou seja,

$$
I_{\lambda}\left(u_{n}^{2}\right) \geq m_{\lambda}^{\infty}-\overline{\varepsilon_{n}} .
$$


Para $\left(u_{n}^{1}\right)$, usaremos $F(x, s) \leq \frac{1}{2} f(x, s) s^{2}$ e $(2.44)$, assim

$$
\begin{aligned}
I_{\lambda}\left(u_{n}^{1}\right) & =\frac{1}{2}\left\|u_{n}^{1}\right\|_{\lambda}^{2}-\int F\left(x, u_{n}^{1}\right) d x \\
& \geq \frac{1}{2} \int f\left(x, u_{n}^{1}\right)\left(u_{n}^{1}\right)^{2} d x-\overline{\varepsilon_{n}}-\int F\left(x, u_{n}^{1}\right) d x \\
& \geq \frac{1}{2} \int f\left(x, u_{n}^{1}\right)\left(u_{n}^{1}\right)^{2} d x-\int \frac{1}{2} f\left(x, u_{n}^{1}\right)\left(u_{n}^{1}\right)^{2} d x-\overline{\varepsilon_{n}} \\
& =-\overline{\varepsilon_{n}}
\end{aligned}
$$

ou seja,

$$
I_{\lambda}\left(u_{n}^{1}\right) \geq-\overline{\varepsilon_{n}}
$$

Logo, por $(2.55),(2.56)$ e por $I_{\lambda}\left(u_{n}\right) \geq I_{\lambda}\left(u_{n}^{1}\right)+I_{\lambda}\left(u_{n}^{2}\right)-C \varepsilon$, temos

$$
I_{\lambda}\left(u_{n}\right) \geq I_{\lambda}\left(u_{n}^{1}\right)+I_{\lambda}\left(u_{n}^{2}\right)-\overline{\varepsilon_{n}} \geq m_{\lambda}^{\infty}-2 \overline{\varepsilon_{n}},
$$

e assim chegamos em $\lim _{n \rightarrow \infty} I_{\lambda}\left(u_{n}\right) \geq m_{\lambda}^{\infty}$, o que é um absurdo, pois $\lim _{n \rightarrow \infty} I_{\lambda}\left(u_{n}\right)=c<m_{\lambda}^{\infty}$. Portanto, a dicotomia não ocorre.

Caso 2: $\left(y_{n}\right) \subset \mathbb{R}^{N}$ não é limitado.

Passando a uma subsequência se necessário, podemos assumir que $\left|y_{n}\right| \rightarrow \infty$. Pela definição de $\left(u_{n}^{1}\right)$, temos que $u_{n}^{1}(x)=0$ quando $\left|x-y_{n}\right| \geq 2 R$ e segue que $\operatorname{supp}\left(u_{n}^{1}\right) \subset B_{2 R}\left(y_{n}\right)$, onde os centros das bolas vão para o infinito.

Repetindo para os mesmos passos feitos no caso anterior com $u_{n}^{1}$ e $u_{n}^{2}$ trocados geramos novamente uma contradição. Portanto, a dicotomia não ocorre e, pelo Lema 2.5 de Concentração e Compacidade de Lions, a compacidade ocorre.

3. Compacidade: Existe $\left(y_{n}\right) \subset \mathbb{R}^{N}$ tal que para todo $\varepsilon>0$, existe $R>0$, tal que

$$
\int_{B_{R}\left(y_{n}\right)} \rho_{n} d x \geq \alpha-\varepsilon
$$

isto é,

$$
\int_{\mathbb{R}^{N} \backslash B_{R}\left(y_{n}\right)} \rho_{n} d x=\int \rho_{n} d x-\int_{B_{R}\left(y_{n}\right)} \rho_{n} d x \leq \varepsilon,
$$

ou seja,

$$
\int_{\mathbb{R}^{N} \backslash B_{R}\left(y_{n}\right)}\left(\left|\nabla u_{n}\right|^{2}+\lambda\left|u_{n}\right|^{2}\right) d x<\varepsilon .
$$

Como no caso da dicotomia, podemos mostrar que se (para alguma subsequência) $\left|y_{n}\right| \rightarrow \infty$, temos uma contradição com $I_{\lambda}\left(u_{n}\right) \rightarrow c<m_{\lambda}^{\infty}$ (note que se $c=m_{\lambda}^{\infty}$ e $f(x, s)=h(s)$ é independente de $x$, então $\left|y_{n}\right| \rightarrow \infty$ não pode ser descartado ). Portanto, $\left(y_{n}\right) \subset \mathbb{R}^{N}$ é uma sequência limitada e, para todo $\varepsilon>0$, podemos achar $R_{0}>0$ grande, tal que

$$
B_{R}\left(y_{n}\right) \subset B_{R_{0}}(0) .
$$


De $(2.57)$, temos

$$
\int_{\mathbb{R}^{N} \backslash B_{R_{0}}}\left(\left|\nabla u_{n}\right|^{2}+\lambda\left|u_{n}\right|^{2}\right) d x \leq \int_{\mathbb{R}^{N} \backslash B_{R}\left(y_{n}\right)}\left(\left|\nabla u_{n}\right|^{2}+\lambda\left|u_{n}\right|^{2}\right) d x<\varepsilon .
$$

Como $\left(u_{n}\right)$ é limitada em $H^{1}\left(\mathbb{R}^{N}\right)$, podemos assumir que

$$
u_{n} \rightarrow u, \text { em } H^{1}\left(\mathbb{R}^{N}\right)
$$

Mostraremos que $u_{n} \rightarrow u$ em $L^{p}\left(\mathbb{R}^{N}\right)$, para $2 \leq p<2^{*}$. De fato, sendo $|u|^{p}$ integrável, para todo $\varepsilon>0$, tome $\bar{R}(\varepsilon)=\bar{R}>0$ grande de forma que

$$
\int_{\mathbb{R}^{N} \backslash B_{\bar{R}}}|u|^{p} d x \leq \varepsilon
$$

Portanto, se $R^{*}=\max \left\{R_{0}, \bar{R}\right\}$, então por (2.58), (2.59) e as imersões de Sobolev, temos

$$
\begin{aligned}
\int\left|u_{n}-u\right|^{p} d x & =\int_{B_{R^{*}}}\left|u_{n}-u\right|^{p} d x+\int_{\mathbb{R}^{N} \backslash B_{R^{*}}}\left|u_{n}-u\right|^{p} d x \\
& \leq \varepsilon+2^{p}\left(\int_{\mathbb{R}^{N} \backslash B_{R^{*}}}\left|u_{n}\right|^{p} d x+\int_{\mathbb{R}^{N} \backslash B_{R^{*}}}|u|^{p} d x\right) \\
& \leq C \varepsilon .
\end{aligned}
$$

Logo, $u_{n} \rightarrow u$ em $L^{p}\left(\mathbb{R}^{N}\right)$, para $2 \leq p<2^{*}$.

Assim, $u_{n} \rightarrow u$, em quase todo ponto de $\mathbb{R}^{N}$, e existe $\tilde{h} \in L^{2}\left(\mathbb{R}^{N}\right)$ tal que

$$
\left|u_{n}\right| \leq \tilde{h}, \text { em } \mathbb{R}^{N}
$$

Desta forma

$$
f\left(x, u_{n}\right)\left(u_{n}\right)^{2} \leq g \tilde{h}^{2} \in L^{1}\left(\mathbb{R}^{N}\right)
$$

e

$$
\left|f\left(x, u_{n}\right) u_{n} u\right| \leq g \tilde{h}|u| \in L^{1}\left(\mathbb{R}^{N}\right) .
$$

Pelo Teorema 1.10 da Convergência Dominada de Lebesgue, temos

$$
\int f\left(x, u_{n}\right)\left(u_{n}\right)^{2} d x \rightarrow \int f(x, u) u^{2} d x
$$

e

$$
\int f\left(x, u_{n}\right) u_{n} u d x \rightarrow \int f(x, u) u^{2} d x
$$

De maneira análoga, obtemos

$$
\int f(x, u) u_{n} u d x \rightarrow \int f(x, u) u^{2} d x
$$


Desta forma, $u_{n} \rightarrow u$, em $H^{1}\left(\mathbb{R}^{N}\right)$. De fato

$$
\begin{aligned}
I_{\lambda}^{\prime}\left(u_{n}\right)\left(u_{n}-u\right)-I_{\lambda}^{\prime}(u)\left(u_{n}-u\right) & =\int\left(\nabla u_{n} \nabla\left(u_{n}-u\right)+\lambda u_{n}\left(u_{n}-u\right)\right) d x \\
& -\int f\left(x, u_{n}\right) u_{n}\left(u_{n}-u\right) d x-\int\left(\nabla u \nabla\left(u_{n}-u\right)+\lambda u\left(u_{n}-u\right) d x\right. \\
& +\int f(x, u) u\left(u_{n}-u\right) d x \\
& =\int\left(\left|\nabla\left(u_{n}-u\right)\right|^{2}+\lambda\left(u_{n}-u\right)^{2}\right) d x \\
& -\int f\left(x, u_{n}\right)\left(u_{n}\right)^{2} d x+\int f\left(x, u_{n}\right) u_{n} u d x \\
& +\int f(x, u) u u_{n} d x-\int f(x, u) u^{2} d x .
\end{aligned}
$$

Logo

$$
\begin{aligned}
\left\|u_{n}-u\right\|_{\lambda}^{2} & =I_{\lambda}^{\prime}\left(u_{n}\right)\left(u_{n}-u\right)-I_{\lambda}^{\prime}(u)\left(u_{n}-u\right) \\
& +\int f\left(x, u_{n}\right)\left(u_{n}\right)^{2} d x+\int f(x, u) u^{2} d x \\
& -\int f\left(x, u_{n}\right) u_{n} u d x-\int f(x, u) u u_{n} d x .
\end{aligned}
$$

Como $I_{\lambda}^{\prime}\left(u_{n}\right)\left(u_{n}-u\right) \rightarrow 0$ e $I_{\lambda}^{\prime}(u)\left(u_{n}-u\right) \rightarrow 0$, já que $u_{n} \rightarrow u$ em $H^{1}\left(\mathbb{R}^{N}\right)$, então de (2.60) - (2.62) segue que

$$
\left\|u_{n}-u\right\|_{\lambda}^{2} \rightarrow 0
$$

Portanto, a sequência de Cerami $\left(u_{n}\right)$ possui uma subsequência convergente e o resultado segue.

Observação 2.4. Como já foi destacado na prova do Teorema 2.2 acima, a mesma prova implica no seguinte resultado para o caso de $f(x, s)$ ser independente de $x$ : "Se $\left(u_{n}\right)$ é uma sequência de Cerami para $I_{\lambda}$ no nivel $c=m_{\lambda}^{\infty}$, então existe $\left(y_{n}\right) \subset \mathbb{R}^{N}$ tal que $\tilde{u}_{n}(\cdot)=u_{n}\left(\cdot+y_{n}\right)$ tem uma subsequência convergente." 


\section{Capítulo}

\section{Existência de Solução Positiva}

Vamos começar considerando as condições da geometria do Passo da Montanha ( (a) e (b) do Teorema 2.1).

\subsection{Geometria do Passo da Montanha}

Proposição 3.1. Assuma as condições $\left(f_{1}\right)-\left(f_{4}\right)$ e $0<\lambda<|\Lambda|$. Então:

(a) Existem $\rho(\lambda), \alpha(\lambda)>0$ tais que $I_{\lambda}(u) \geq \alpha(\lambda)$ se $\|u\|_{\lambda}=\rho(\lambda)$ e $I_{\lambda}(u) \geq 0$ para $\|u\|_{\lambda} \leq \rho(\lambda)$;

(b) Existe $e_{\lambda} \in H^{1}\left(\mathbb{R}^{N}\right)$ tal que $\left\|e_{\lambda}\right\|_{\lambda}>\rho(\lambda)$ e $I_{\lambda}\left(e_{\lambda}\right) \leq 0$.

Demonstração. (a) Este argumento é padrão. Utilizando (2.21) e (2.22), seja $\varepsilon=\frac{1}{4} \min \{1, \lambda\}$ e tome $C_{\varepsilon}>0$, tal que

$$
0 \leq F(x, s) \leq \frac{1}{2} f(x, s) s^{2} \leq \varepsilon s^{2}+C\left(\varepsilon, 2^{*}\right) s^{2^{*}} .
$$

Segue da imersão de Sobolev $H^{1}\left(\mathbb{R}^{N}\right) \hookrightarrow L^{2^{*}}\left(\mathbb{R}^{N}\right)$ que

$$
\int F(x, u) d x \leq \varepsilon\|u\|_{2}^{2}+C_{\varepsilon}\|u\|_{2^{*}}^{2^{*}} \leq \varepsilon\|u\|_{2}^{2}+C\|u\|_{\lambda}^{2^{*}},
$$

onde $C=C(\varepsilon, N)$. Como $\frac{1}{4} \min \{1, \lambda\}=\varepsilon \leq \frac{1}{4} \lambda$, então chegamos em

$$
\varepsilon\|u\|_{2}^{2} \leq \frac{1}{4}\left[\lambda \int u^{2} d x\right] \leq \frac{1}{4}\left[\int|\nabla u|^{2} d x+\lambda \int u^{2} d x\right]=\frac{1}{4}\|u\|_{\lambda}^{2},
$$

e assim

$$
\int F(x, u) d x \leq \frac{1}{4}\|u\|_{\lambda}^{2}+C\|u\|_{\lambda}^{2^{*}}
$$


Portanto, para qualquer $u \in H^{1}\left(\mathbb{R}^{N}\right)$, obtemos a estimativa inferior

$$
\begin{aligned}
I_{\lambda}(u) & =\frac{1}{2}\|u\|_{\lambda}^{2}-\int F(x, u) d x \\
& \geq \frac{1}{2}\|u\|_{\lambda}^{2}-\frac{1}{4}\|u\|_{\lambda}^{2}-C\|u\|_{\lambda}^{2^{*}} \\
& =\frac{1}{4}\|u\|_{\lambda}^{2}-C\|u\|_{\lambda}^{2^{*}} \\
& =\left(\frac{1}{4}-C\|u\|_{\lambda}^{2^{*}-2}\right)\|u\|_{\lambda}^{2}
\end{aligned}
$$

ou seja,

$$
I_{\lambda}(u) \geq\left(\frac{1}{4}-C\|u\|_{\lambda}^{2^{*}-2}\right)\|u\|_{\lambda}^{2}
$$

e para $\|u\|_{\lambda}=\rho$ suficientemente pequeno, tal que $\left(\frac{1}{4}-C\|u\|_{\lambda}^{2^{*}-2}\right)$ seja positivo, teremos $I_{\lambda}(u) \geq \alpha>0$, o que prova $(\mathrm{a})$.

(b) Dado $0 \neq u \in H^{1}\left(\mathbb{R}^{N}\right)$, vamos considerar a função $p: \mathbb{R}^{+} \rightarrow \mathbb{R}$ definida por

$$
p(t)=I_{\lambda}(t u)=\frac{1}{2} t^{2}\|u\|_{\lambda}^{2}-\int F(x, t u) d x .
$$

Então, temos

$$
p^{\prime}(t)=I_{\lambda}^{\prime}(t u) u=t\left[\|u\|_{\lambda}^{2}-\int f(x, t u) u^{2} d x\right]
$$

Como em (a), temos

$$
p(t) \geq\left(\frac{1}{4}-C\left(t\|u\|_{\lambda}\right)^{2^{*}-2}\right) t^{2}\|u\|_{\lambda}^{2}
$$

$$
p^{\prime}(t) \geq t\|u\|_{\lambda}-\frac{1}{4} t^{2}\|u\|_{\lambda}^{2}-C t^{2}\|u\|_{\lambda}^{2^{*}}
$$

e os mesmos argumentos da parte (a) mostram que $p(t)>0, p^{\prime}(t)>0$ para $t>0$ pequeno. Provaremos a seguir as seguintes afirmações.

Afirmação 1: $\lim _{t \rightarrow \infty} \frac{1}{t^{2}} \int F(x, t u) d x=\frac{1}{2} \int g(x)\left(u^{+}\right)^{2} d x ;$

Afirmação 2: $\lim _{t \rightarrow \infty} \int f(x, t u) u^{2} d x=\int g(x)\left(u^{+}\right)^{2} d x$.

Para provar a Afirmação 2, basta aplicar o Teorema 1.10 da Convergência Dominada de Lebesgue. Vamos provar apenas a Afirmação 1. Para isso, note que

$$
\begin{aligned}
\int F(x, t u) d x & =\int\left(\int_{0}^{t u} f(x, s) s d s\right) d x \\
& =\int\left(t^{2} u^{2} \int_{0}^{1} f(x, \tau t u) \tau d \tau\right) d x
\end{aligned}
$$

onde na última igualdade fizemos a mudança de variáveis $s=\tau t u$, com $d s=t u d \tau$.

Para todo $\varepsilon>0$, pela hipótese $\left(f_{2}\right)$ existe $\bar{t}$, tal que

$$
|f(x, t \tau u(x))-g(x)|<\varepsilon, \quad \forall t>\bar{t} \text { e } x \in \mathbb{R}^{N}
$$


Logo

$$
\left|\int_{0}^{1}[f(x, \tau t u)-g(x)] \tau d \tau\right|<\int_{0}^{1} \varepsilon \tau d \tau=\frac{1}{2} \varepsilon<\varepsilon, \quad \forall t>\bar{t} .
$$

Assim, temos, para $t \rightarrow \infty$, que

$$
\begin{aligned}
u^{2} \int_{0}^{1} f(x, \tau t u(x)) \tau d \tau & \rightarrow\left(u^{+}\right)^{2} \int_{0}^{1} g(x) \tau d \tau \\
& =\frac{1}{2}\left(u^{+}\right)^{2} g(x), \text { em quase todo ponto de } \mathbb{R}^{N}
\end{aligned}
$$

Por outro lado, do fato que $f(x, s) \leq g(x)$, temos

$$
\begin{aligned}
u^{2} \int_{0}^{1} f(x, \tau t u) \tau d \tau & \leq u^{2} \int_{0}^{1} g(x) \tau d \tau \\
& \leq \frac{1}{2} u^{2} g(x) \in L^{1}\left(\mathbb{R}^{N}\right)
\end{aligned}
$$

Logo, pelo Teorema 1.10 da Convergência Dominada de Lebesgue e por $\left(f_{2}\right)$, temos

$$
\begin{aligned}
\lim _{t \rightarrow \infty} \frac{1}{t^{2}} \int F(x, t u) d x & =\lim _{t \rightarrow \infty} \int\left(u^{2} \int_{0}^{1} f(x, \tau t u) \tau d \tau\right) d x \\
& =\int\left(u^{2} \int_{0}^{1} \lim _{t \rightarrow \infty} f(x, \tau t u) \tau d \tau\right) d x \\
& =\int\left(u^{2} g(x) \int_{0}^{1} \tau d \tau\right) d x \\
& =\frac{1}{2} \int g(x)\left(u^{+}\right)^{2} d x
\end{aligned}
$$

Agora, como $\left(f_{2}\right)$ faz com que $f(x, s)$ seja uma função não-decrescente em $s,(3.2)$ nos mostra que $\frac{p^{\prime}(t)}{t}$ é uma função não-crescente em $t$ e a Afirmação 2 implica que

$$
\lim _{t \rightarrow \infty} \frac{p^{\prime}(t)}{t}=\lim _{t \rightarrow \infty}\left[\|u\|_{\lambda}^{2}-\int f(x, t u) u^{2} d x\right]=\|u\|_{\lambda}^{2}-\int g(x)\left(u^{+}\right)^{2} d x
$$

Similarmente, obtemos de (3.1) e da Afirmação 1 que

$$
\lim _{t \rightarrow \infty} \frac{p(t)}{t^{2}}=\lim _{t \rightarrow \infty}\left[\frac{1}{2}\|u\|_{\lambda}^{2}-\frac{1}{t^{2}} \int F(x, t u) d x\right]=\frac{1}{2}\left(\|u\|_{\lambda}^{2}-\int g(x)\left(u^{+}\right)^{2} d x\right) .
$$

Suponha que $\|u\|_{\lambda}^{2}-\int g(x)\left(u^{+}\right)^{2} d x \geq 0, \operatorname{logo} \lim _{t \rightarrow \infty} \frac{p^{\prime}(t)}{t} \geq 0$ e portanto devemos ter $p^{\prime}(t) \geq 0$, para todo $t>0$, pois, caso contrário, existiria um $\tilde{t}>0$ tal que $p^{\prime}(\tilde{t})<0$ e por $\frac{p^{\prime}(t)}{t}$ ser não-crescente, teríamos

$$
\frac{p^{\prime}(t)}{t} \leq \frac{p^{\prime}(\tilde{t})}{\tilde{t}}<0, \quad \text { para todo } t \geq \tilde{t}
$$

e assim $\lim _{t \rightarrow \infty} \frac{p^{\prime}(t)}{t}<0$, um absurdo. Logo $p^{\prime}(t) \geq 0$ e portanto $p(t)$ é não-decrescente. Desde que $p(t)>0$, para todo $t>0$ pequeno, devemos ter $p(t)=I_{\lambda}(t u)>0$, para todo $t>0$.

Suponha agora que $\|u\|_{\lambda}^{2}-\int g(x)\left(u^{+}\right)^{2} d x<0$, e como $p^{\prime}(t)>0$, para $t>0$ pequeno, então $p^{\prime}(t)$ 
muda de sinal. Portanto existe $t_{1}>0$ tal que $p^{\prime}\left(t_{1}\right)=0$. Defina $w_{0}=\left\{t>0 \mid t \leq t_{1}\right.$ e $\left.p^{\prime}(t)=0\right\}$ e $t_{0}=\inf w_{0}$. Sendo $\frac{p^{\prime}(t)}{t}$ não crescente em $t$, temos que, se $t<t_{0}$, então

$$
\frac{p^{\prime}(t)}{t} \geq \frac{p^{\prime}\left(t_{0}\right)}{t_{0}} \geq \frac{p^{\prime}\left(t_{1}\right)}{t_{1}}=0
$$

Portanto $p^{\prime}(t) \geq 0$ para todo $t<t_{0}$. Por outro lado, se $p^{\prime}(t)=0$, teremos $t \in w_{0}$ e $t<t_{0}$, uma contradição, logo $p^{\prime}(t)>0$ para todo $t<t_{0}$.

Agora definimos $t_{2}=\sup w_{1}$, onde $w_{1}=\left\{t>0 \mid t \geq t_{1}\right.$ e $\left.p^{\prime}(t)=0\right\}$, e teremos que $p^{\prime}(t)<0$, para todo $t>t_{2}$. Desta forma, existem $t_{0} \leq t_{2} \operatorname{com} t_{0}=t_{0}(u), t_{2}=t_{2}(u)$, tais que

$$
\begin{cases}p^{\prime}(t)>0, & \text { para } t<t_{0} \\ p^{\prime}(t)=0, & \text { para } t_{0} \leq t \leq t_{2} \\ p^{\prime}(t)<0, & \text { para } t>t_{2}\end{cases}
$$

Em particular, temos

$$
0=t_{0} p^{\prime}\left(t_{0}\right)=I_{\lambda}^{\prime}\left(t_{0} u\right)\left(t_{0} u\right)=\left\|t_{0} u\right\|_{\lambda}^{2}-\int f\left(x, t_{0} u\right)\left(t_{0} u\right)^{2} d x
$$

Além disso,

$$
\max _{0<t<\infty} p(t)=\max _{0<t<\infty} I_{\lambda}(t u)=I_{\lambda}(\bar{t} u), \quad \forall \bar{t} \in\left[t_{0}, t_{2}\right] \quad \text { e } \quad \lim _{t \rightarrow \infty} I_{\lambda}(t u)=-\infty .
$$

Agora vamos considerar os dois possíveis casos:

Caso $1 . \Lambda<-l_{\infty}$.

Neste caso, como visto na demonstração do Lema $2.4, \Lambda$ é o menor autovalor de $S$. Seja $\psi>0$ uma autofunção associada a $\Lambda$, temos

$$
\int|\nabla \psi|^{2} d x-\int g(x) \psi^{2} d x=\Lambda \int \psi^{2} d x
$$

portanto

$$
\int\left(|\nabla \psi|^{2}+\lambda \psi^{2}\right) d x-\int g(x) \psi^{2} d x=(\lambda+\Lambda) \int \psi^{2} d x<0
$$

já que $\lambda+\Lambda<0$, uma vez que $\lambda<|\Lambda|$. Portanto, em vista de (3.4), temos que $\lim _{t \rightarrow \infty} I_{\lambda}(t \psi)=-\infty$ de modo que podemos tomar $e_{\lambda}=t \psi$, para algum $\mathrm{t}$ suficientemente grande, e chegamos em $I_{\lambda}\left(e_{\lambda}\right) \leq 0$.

Caso 2. $\Lambda=-l_{\infty}$.

Neste caso temos $\lambda<|\Lambda|=l_{\infty}$. Agora, se tomarmos $0 \leq \phi \in C_{0}^{\infty}\left(\mathbb{R}^{N} \backslash B_{1}\right)$, onde $B_{1}$ é a bola unitária de $\mathbb{R}^{N}$, e definirmos $\phi_{\sigma}(x)=\sigma^{N / 2} \phi(\sigma x)$, então, usando mudança de variáveis e o Teorema 1.10 da Convergência Dominada de Lebesgue, temos

$$
\begin{aligned}
\int g(x) \phi_{\sigma}^{2}(x) d x & =\int \sigma^{N} g(x) \phi^{2}(\sigma x) d x \\
& =\int_{\mathbb{R}^{N} \backslash B_{1}} g\left(\frac{x}{\sigma}\right) \phi^{2}(x) d x \rightarrow l_{\infty} \int \phi^{2}(x) d x,
\end{aligned}
$$


quando $\sigma \rightarrow 0$. Como

$$
\begin{array}{rlrl}
\frac{\partial \phi_{\sigma}(x)}{\partial x_{i}}=\frac{\partial\left(\sigma^{N / 2} \phi(\sigma x)\right)}{\partial x_{i}} & =\sigma^{N / 2} \frac{\partial \phi(\sigma x)}{\partial x_{i}} \sigma & \Rightarrow \\
\nabla \phi_{\sigma}(x) & =\sigma^{N / 2} \sigma \nabla \phi(\sigma x) & \Rightarrow \\
\left|\nabla \phi_{\sigma}(x)\right|^{2} & =\sigma^{N} \sigma^{2}|\nabla \phi(\sigma x)|^{2} & \Rightarrow \\
\int\left|\nabla \phi_{\sigma}(x)\right|^{2} d x & =\sigma^{N} \sigma^{2} \int|\nabla \phi(\sigma x)|^{2} d x,
\end{array}
$$

e fazendo a mudança de variável $y=\sigma x$, obtemos

$$
\left\|\nabla \phi_{\sigma}\right\|_{2}^{2}=\sigma^{2}\|\nabla \phi\|_{2}^{2} \rightarrow 0, \text { quando } \sigma \rightarrow 0
$$

Além disso, note que

$$
\begin{aligned}
\left\|\phi_{\sigma}\right\|_{2}^{2} & =\int\left(\phi_{\sigma}(x)\right)^{2} d x \\
& =\int\left(\sigma^{N / 2} \phi(\sigma x)\right)^{2} d x \\
& =\sigma^{N} \int(\phi(\sigma x))^{2} d x \\
& =\int(\phi(x))^{2} d x \\
& =\|\phi\|_{2}^{2},
\end{aligned}
$$

e assim, obtemos

$$
\lim _{\sigma \rightarrow 0}\left(\left\|\phi_{\sigma}\right\|_{\lambda}^{2}-\int g(x) \phi_{\sigma}^{2}(x) d x\right)=\left(\lambda-l_{\infty}\right) \int \phi^{2}(x) d x<0 .
$$

Portanto, para $\sigma>0$ suficientemente pequeno, temos $\left\|\phi_{\sigma}\right\|_{\lambda}^{2}-\int g(x) \phi_{\sigma}^{2}(x) d x<0$ de modo que, em vista de (3.4), podemos novamente tomar $e_{\lambda}=t \phi_{\sigma}$, com $t$ grande, para chegar em $I_{\lambda}\left(e_{\lambda}\right) \leq 0$. Isto completa a prova.

Observação 3.1. Se $f(x, s)=h(s)$, ou seja, $f$ independente de $x$, então $\Lambda=-l_{\infty}$. Uma vez que $g(x)=1$ e $S_{0}=-\Delta-1$, logo temos o seguinte problema de autovalor

$$
-\Delta u=(1+\mu) u, \quad \text { em } \mathbb{R}^{N}
$$

que não possui solução. Portanto,

$$
\sigma(S)=\sigma_{e s s}(S)=\left[-l_{\infty}, \infty\right)
$$

e desde que $\Lambda=\inf \sigma(S)$ temos $\Lambda=-l_{\infty}$.

Como consequência da Proposição 3.1, temos os seguintes resultados.

Proposição 3.2. Assuma $\left(f_{1}\right)-\left(f_{4}\right)$ e suponha que $0<\lambda<|\Lambda|$. Defina

$$
M_{\lambda}=\left\{u \neq 0 \mid I_{\lambda}^{\prime}(u) u=\|u\|_{\lambda}^{2}-\int f(x, u) u^{2} d x=0\right\}
$$


e tome $u \in H^{1}\left(\mathbb{R}^{N}\right)$.

(a) $S e\|u\|_{\lambda}^{2}-\int g(x)\left(u^{+}\right)^{2} d x \geq 0$, então $\mathbb{R}^{+} u \cap M_{\lambda}=\varnothing$ e $I_{\lambda}(t u)>0, \quad \forall t>0 ;$

(b) Se $\|u\|_{\lambda}^{2}-\int g(x)\left(u^{+}\right)^{2} d x<0$, então existem $0<t_{0}(u) \leq t_{2}(u)$ tais que $\bar{t} u \in M_{\lambda}$, para $\bar{t} \in\left[t_{0}, t_{2}\right]$ $e$

$$
\max _{0<t<\infty} I_{\lambda}(t u)=I_{\lambda}(\bar{t} u), \quad \forall \bar{t} \in\left[t_{0}, t_{2}\right] \quad e \quad \lim _{t \rightarrow \infty} I_{\lambda}(t u)=-\infty .
$$

Observação 3.2. Ficou claro das provas acima que, caso $f(x, s)=h(s)$ seja independente de $x$, os resultados da Proposição 3.1 e 3.2 ainda são válidos $\operatorname{com} M_{\lambda}, I_{\lambda}$ e $g(x)$ sendo substituídos por $M_{\lambda}^{\infty}, I_{\lambda}^{\infty}$, e $l_{\infty}$, respectivamente.

Agora estamos prontos para mostrar a existência de soluções positivas para $\left(P_{\lambda}\right)$.

\subsection{Existência de Solução Positiva}

Vamos considerar primeiramente o problema no infinito:

$$
\left(P_{\infty}\right) \quad-\Delta u+\lambda u=h(u) u, \quad u \in H^{1}\left(\mathbb{R}^{N}\right), \quad u>0 .
$$

Provamos a existência de uma solução para $\left(P_{\infty}\right)$ aplicando o Teorema do Passo da Montanha (Teorema 2.1) ao funcional correspondente $I_{\lambda}^{\infty}$ definido em (2.23):

$$
I_{\lambda}^{\infty}(u)=\frac{1}{2}\|u\|_{\lambda}^{2}-\int H(u) d x, \quad u \in H^{1}\left(\mathbb{R}^{N}\right) .
$$

De fato, pela Proposição 3.1 (veja Observação 3.2), sabemos que $I_{\lambda}^{\infty}$ satisfaz as condições (a) e (b) do Teorema 2.1 do Passo da Montanha e, pelo Teorema 2.2 (veja Observação 2.4), o funcional satisfaz uma forma adequada da condição de compacidade de Cerami em todos os níveis $c$ tais que $0<c \leq m_{\lambda}^{\infty}$. Vamos considerar o nível

$$
0<c_{\lambda}^{\infty}:=\inf _{\gamma \in \Gamma_{\infty}} \sup _{0 \leq t \leq 1} I_{\lambda}^{\infty}(\gamma(t))
$$

onde

$$
\Gamma_{\infty}=\left\{\gamma \in C\left([0,1], H^{1}\left(\mathbb{R}^{N}\right)\right) \mid \gamma(0)=0, I_{\lambda}^{\infty}(\gamma(1))<0\right\} .
$$

Vamos mostrar em seguida que $c_{\lambda}^{\infty} \leq m_{\lambda}^{\infty}$, de modo que $c_{\lambda}^{\infty}$ é um valor crítico de $I_{\lambda}^{\infty}$. Note que, neste caso, teremos necessariamente $c_{\lambda}^{\infty}=m_{\lambda}^{\infty}$, já que se temos $u_{0}$ solução de $\left(P_{\infty}\right)$ então $u_{0}$ é ponto crítico de $I_{\lambda}^{\infty} \mathrm{e}$ qualquer ponto crítico de $I_{\lambda}^{\infty}$ pertence ao conjunto $M_{\lambda}^{\infty}=\left\{u \neq 0 \mid I_{\lambda}^{\infty \prime}(u) u=\|u\|_{\lambda}^{2}-\int h(u) u d x=0\right\}$, $\log 0$

$$
c_{\lambda}^{\infty}=I_{\lambda}^{\infty}\left(u_{0}\right) \geq \inf _{u \in M_{\lambda}^{\infty}} I_{\lambda}^{\infty}(u)=m_{\lambda}^{\infty} .
$$

Agora, dado $u \in M_{\lambda}^{\infty}$ e pela Proposição 3.2 e a Observação 3.2, temos que

$$
\|u\|_{\lambda}^{2}-l_{\infty} \int\left(u^{+}\right)^{2} d x<0, \quad I_{\lambda}^{\infty}(t u) \leq 0, \quad \text { para } t \geq t_{2}(u),
$$

e

$$
\max _{0<t<\infty} I_{\lambda}^{\infty}(t u)=I_{\lambda}^{\infty}\left(t_{2} u\right) \leq 0
$$


Portanto se considerarmos $\tilde{\gamma}:[0,1] \rightarrow H^{1}\left(\mathbb{R}^{N}\right), \tilde{\gamma}(s)=s t_{2} u$, então $\tilde{\gamma} \in \Gamma_{\infty}$ e

$$
c_{\lambda}^{\infty} \leq \sup _{0 \leq s \leq 1} I_{\lambda}^{\infty}(\tilde{\gamma}(s)) \leq \sup _{0<t<\infty} I_{\lambda}^{\infty}(t u)=I_{\lambda}^{\infty}\left(t_{2} u\right)
$$

Como $t_{2} u \in M_{\lambda}^{\infty}$, e $u$ é arbitrário, concluímos que $c_{\lambda}^{\infty} \leq \inf _{u \in M_{\lambda}^{\infty}} I_{\lambda}^{\infty}(u)=m_{\lambda}^{\infty}$. Logo, aplicando o Teorema 2.1 do Passo da Montanha, temos a existência da solução $u_{0}$ de $\left(P_{\infty}\right)$ no nível $c_{\lambda}^{\infty}=m_{\lambda}^{\infty}$ :

$$
I_{\lambda}^{\infty}\left(u_{0}\right)=c_{\lambda}^{\infty}=m_{\lambda}^{\infty}
$$

Finalmente, mostraremos que $\left(P_{\lambda}\right)$ tem solução positiva para qualquer $0<\lambda<|\Lambda|$.

Teorema 3.1. Assuma $\left(f_{1}\right)-\left(f_{5}\right)$ e $0<\lambda<|\Lambda|$. Então $\left(P_{\lambda}\right)$ tem uma solução positiva.

Demonstração. Como feito acima, usando o Teorema 2.2 e a Proposição 3.1 vemos que $I_{\lambda}$ satisfaz as condições do Teorema 2.1 do Passo da Montanha com a condição de Cerami sendo satisfeita em todos os níveis $c \in\left(0, m_{\lambda}^{\infty}\right)$. Então, seja

$$
\Gamma=\left\{\gamma \in C\left([0,1], H^{1}\left(\mathbb{R}^{N}\right)\right) \mid \gamma(0)=0, I_{\lambda}(\gamma(1))<0\right\}
$$

temos que

$$
0<c_{\lambda}:=\inf _{\gamma \in \Gamma} \sup _{0 \leq t \leq 1} I_{\lambda}(\gamma(t))
$$

é um valor crítico para $I_{\lambda}$ desde que a gente possa mostrar que $c_{\lambda}<m_{\lambda}^{\infty}$. É agora que usamos a condição $\left(f_{5}\right)$ pela primeira vez.

De fato, por $\left(f_{5}\right)$ temos que $h(s) \leq f(x, s), \forall x \in \mathbb{R}^{N}$ e $\forall s \in \mathbb{R}^{+}, \operatorname{logo}$

$$
\begin{array}{rlrl}
h(s) s & \leq f(x, s) s & \Rightarrow \\
\int_{0}^{u} h(\tau) \tau d \tau & \leq \int_{0}^{u} f(x, \tau) \tau d \tau & \Leftrightarrow \\
H(u) & \leq F(x, u) & & \Rightarrow \\
\int H(u) d x & \leq \int F(x, u) d x & & \Rightarrow \\
\frac{1}{2}\|u\|_{\lambda}^{2}-\int F(x, u) d x & \leq \frac{1}{2}\|u\|_{\lambda}^{2}-\int H(u) d x & \Leftrightarrow \\
I_{\lambda}(u) & \leq I_{\lambda}^{\infty}(u), &
\end{array}
$$

para todo $u \in H^{1}\left(\mathbb{R}^{N}\right)$.

Seja $u_{0}$ a solução de $\left(P_{\infty}\right)$, logo $u_{0}$ é ponto crítico de $I_{\lambda}^{\infty}$ e como o conjunto dos pontos críticos de $I_{\lambda}^{\infty}$ está contido em $M_{\lambda}^{\infty}=\left\{u \neq 0 \mid I_{\lambda}^{\infty \prime}(u) u=\|u\|_{\lambda}^{2}-\int h(u) u^{2} d x=0\right\}$, então $u_{0} \in M_{\lambda}^{\infty}$. Além disso, pela Proposição 3.2 (veja Observação 3.2 ), devemos ter $I_{\lambda}^{\infty}\left(t u_{0}\right) \leq 0$, para $t \geq t_{2}\left(u_{0}\right)$, e assim, usando (3.6), teremos

$$
I_{\lambda}\left(t u_{0}\right) \leq I_{\lambda}^{\infty}\left(t u_{0}\right) \leq 0 .
$$

Portanto, se

$$
\begin{aligned}
\bar{\gamma}:[0,1] & \rightarrow H^{1}\left(\mathbb{R}^{N}\right) \\
s & \mapsto \bar{\gamma}=s t_{2} u_{0}
\end{aligned}
$$


teremos $\bar{\gamma}$ contínua, $\bar{\gamma}(0)=0$ e $I_{\lambda}(\bar{\gamma}(1))=I_{\lambda}\left(t_{2} u_{0}\right) \leq 0$ e assim $\bar{\gamma} \in \Gamma$.

Por outro lado, pela Proposição 3.2 (veja Observação 3.2 ), se $u_{0} \in M_{\lambda}^{\infty}$, então necessariamente

$$
\left\|u_{0}\right\|_{\lambda}^{2}-l_{\infty} \int\left(u_{0}^{+}\right)^{2} d x<0
$$

Tomando o limite em $\left(f_{5}\right)$, teremos

$$
g(x)=\lim _{s \rightarrow \infty} f(x, s) \geq \lim _{s \rightarrow \infty} h(s)=l_{\infty}
$$

$\operatorname{logo}$

$$
\begin{array}{rlrl}
-g(x) & \leq-l_{\infty} & \Rightarrow \\
-\int g(x)\left(u_{0}^{+}\right)^{2} d x & \leq-l_{\infty} \int\left(u_{0}^{+}\right)^{2} d x \quad \Leftrightarrow \\
\left\|u_{0}\right\|_{\lambda}^{2}-\int g(x)\left(u_{0}^{+}\right)^{2} d x & \leq\left\|u_{0}\right\|_{\lambda}^{2}-l_{\infty} \int\left(u_{0}^{+}\right)^{2} d x<0
\end{array}
$$

e usando novamente a Proposição 3.2, temos que existe $\bar{t}>0$, tal que $t_{0} \leq \bar{t} \leq t_{2}$ e

$$
\max _{0<t<\infty} I_{\lambda}\left(t u_{0}\right)=I_{\lambda}\left(\bar{t} u_{0}\right)
$$

Ainda usando $\left(f_{5}\right)$ temos que $f(x, s)>h(s)$, para $x \in \omega$, onde $\omega$ tem medida positiva, logo em $\omega$ temos

$$
\begin{aligned}
f(x, t) t & >h(t) t \Rightarrow \\
\int_{0}^{s} f(x, t) t d t & >\int_{0}^{s} h(t) t d t \Leftrightarrow \\
F(x, s) & >H(s) \Rightarrow \\
\int_{\omega} F(x, u) d x & >\int_{\omega} H(u) d x \Rightarrow \\
\int_{\omega} F(x, u) d x+\int_{\mathbb{R}^{N} \backslash \omega} F(x, s) & >\int_{\omega} H(u) d x+\int_{\mathbb{R}^{N} \backslash \omega} H(u) d x \\
\int F(x, u) d x & >\int_{0} H(u) d x \Leftrightarrow \\
-\int F(x, u) d x & <-\int H(u) d x \Leftrightarrow \\
\int_{\lambda}(u) & <I_{\lambda}^{\infty}(u) .
\end{aligned}
$$


Portanto, temos que

$$
\begin{aligned}
c_{\lambda} & \leq \sup _{0 \leq s \leq 1} I_{\lambda}(\bar{\gamma}(s)), & & \text { pela definição de } c_{\lambda} \\
& \leq \sup _{0<t<\infty} I_{\lambda}\left(t u_{0}\right) & & \\
& =I_{\lambda}\left(\bar{t} u_{0}\right), & & \text { por }(3.7) \\
& <I_{\lambda}^{\infty}\left(\bar{t} u_{0}\right), & & \text { por }(3.8) \\
& \leq I_{\lambda}^{\infty}\left(u_{0}\right) & & \\
& =c_{\lambda}^{\infty}=m_{\lambda}^{\infty}, & &
\end{aligned}
$$

onde a última desigualdade segue do fato de que $u_{0} \in M_{\lambda}^{\infty}$. Assim obtemos $c_{\lambda}<m_{\lambda}^{\infty}$, e o Teorema 2.2 juntamente com o Teorema 2.1 do Passo da Montanha provam a existência de uma solução positiva de $\left(P_{\lambda}\right)$.

Encerramos este capítulo com um exemplo de função que satisfaz $\left(f_{1}\right)-\left(f_{5}\right)$.

\subsection{Exemplo de $f$}

Considere a função $f: \mathbb{R}^{N} \times \mathbb{R}^{+} \rightarrow \mathbb{R}$ definida por

$$
f(x, s)=\left(\frac{e^{|x|}+1}{e^{|x|}}\right) \frac{s}{s+1}, \quad x \in \mathbb{R}^{N}, s \in \mathbb{R}^{+},
$$

e vamos mostrar que $f$ satisfaz $\left(f_{1}\right)-\left(f_{5}\right)$.

$\left(f_{1}\right)$ : Claramente $f \in C\left(\mathbb{R}^{N} \times \mathbb{R}^{+}, \mathbb{R}^{+}\right)$. Além disso, como

$$
\begin{aligned}
1 & \leq e^{|x|} \quad \Leftrightarrow \\
e^{|x|}+1 & \leq 2 e^{|x|} \quad \Leftrightarrow \\
\frac{e^{|x|}+1}{e^{|x|}} & \leq 2,
\end{aligned}
$$

então $\frac{e^{|x|}+1}{e^{|x|}}$ é limitado, $\forall x \in \mathbb{R}^{N}$, e assim

$$
|f(x, s)|=\left|\left(\frac{e^{|x|}+1}{e^{|x|}}\right) \frac{s}{s+1}\right| \leq\left|\frac{2 s}{s+1}\right| \rightarrow 0
$$

quando $s \rightarrow 0$. 
$\left(f_{2}\right)$ : Temos, para $x \in \mathbb{R}^{N}$ fixo,

$$
\begin{array}{rlrl}
f(x, s) & \leq f(x, r) & & \Leftrightarrow \\
\left(\frac{e^{|x|}+1}{e^{|x|}}\right) \frac{s}{s+1} & \leq\left(\frac{e^{|x|}+1}{e^{|x|}}\right) \frac{r}{r+1} & \Leftrightarrow \\
\frac{s}{s+1} & \leq \frac{r}{r+1} & & \Leftrightarrow \\
s(r+1) & \leq r(s+1) & \Leftrightarrow \\
s r+s & \leq s r+r & & \Leftrightarrow \\
s & \leq r, &
\end{array}
$$

logo $f(x, \cdot)$ é não-decrescente para $s \in[0, \infty)$. Seja agora

$$
g(x):=\frac{e^{|x|}+1}{e^{|x|}},
$$

$\operatorname{logo} g \in C\left(\mathbb{R}^{N}, \mathbb{R}^{+}\right)$e como $\lim _{s \rightarrow \infty} \frac{s}{s+1}=1$, então

$$
\lim _{s \rightarrow \infty} f(x, s)=\frac{e^{|x|}+1}{e^{|x|}}=g(x) .
$$

$\left(f_{3}\right):$ Seja $h(s):=\frac{s}{s+1}, \log h \in C\left(\mathbb{R}^{+}, \mathbb{R}^{+}\right)$e como $\lim _{|x| \rightarrow \infty} e^{|x|}=\infty$ é equivalente a $\lim _{|x| \rightarrow \infty} e^{-|x|}=0$, então

$$
\begin{aligned}
\lim _{|x| \rightarrow \infty} \frac{e^{|x|}+1}{e^{|x|}} & =\lim _{|x| \rightarrow \infty} \frac{e^{|x|}}{e^{|x|}} \frac{\left(1+e^{-|x|}\right)}{1} \\
& =\lim _{|x| \rightarrow \infty} 1+e^{-|x|}=1,
\end{aligned}
$$

e assim

$$
\lim _{|x| \rightarrow \infty} f(x, s)=\frac{s}{s+1}=h(s) .
$$

$\left(f_{4}\right)$ : Pelos passos acima, temos

$$
\lim _{|x|, s \rightarrow \infty} f(x, s)=1:=l_{\infty} \in(0, \infty)
$$

$\left(f_{5}\right)$ : Como $e^{|x|}+1>e^{|x|}$ se, e somente se, $\frac{e^{|x|}+1}{e^{|x|}}>1$, então

$$
f(x, s)=\left(\frac{e^{|x|}+1}{e^{|x|}}\right) \frac{s}{s+1}>\frac{s}{s+1}=h(s),
$$

para todo $x \in \mathbb{R}^{N}$.

Apresentamos agora os gráficos de $g, h$ e $f$, respectivamente: 


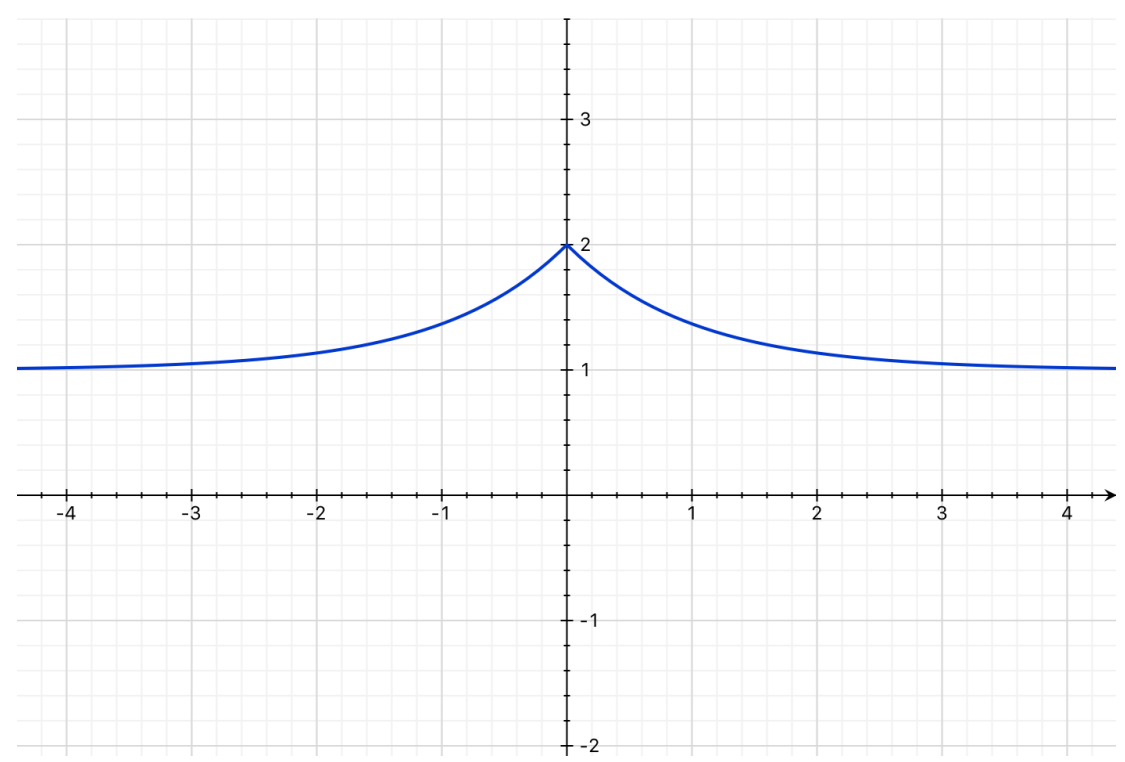

Figura 3.1: Gráfico de $g(x)=\frac{e^{|x|}+1}{e^{|x|}}$.

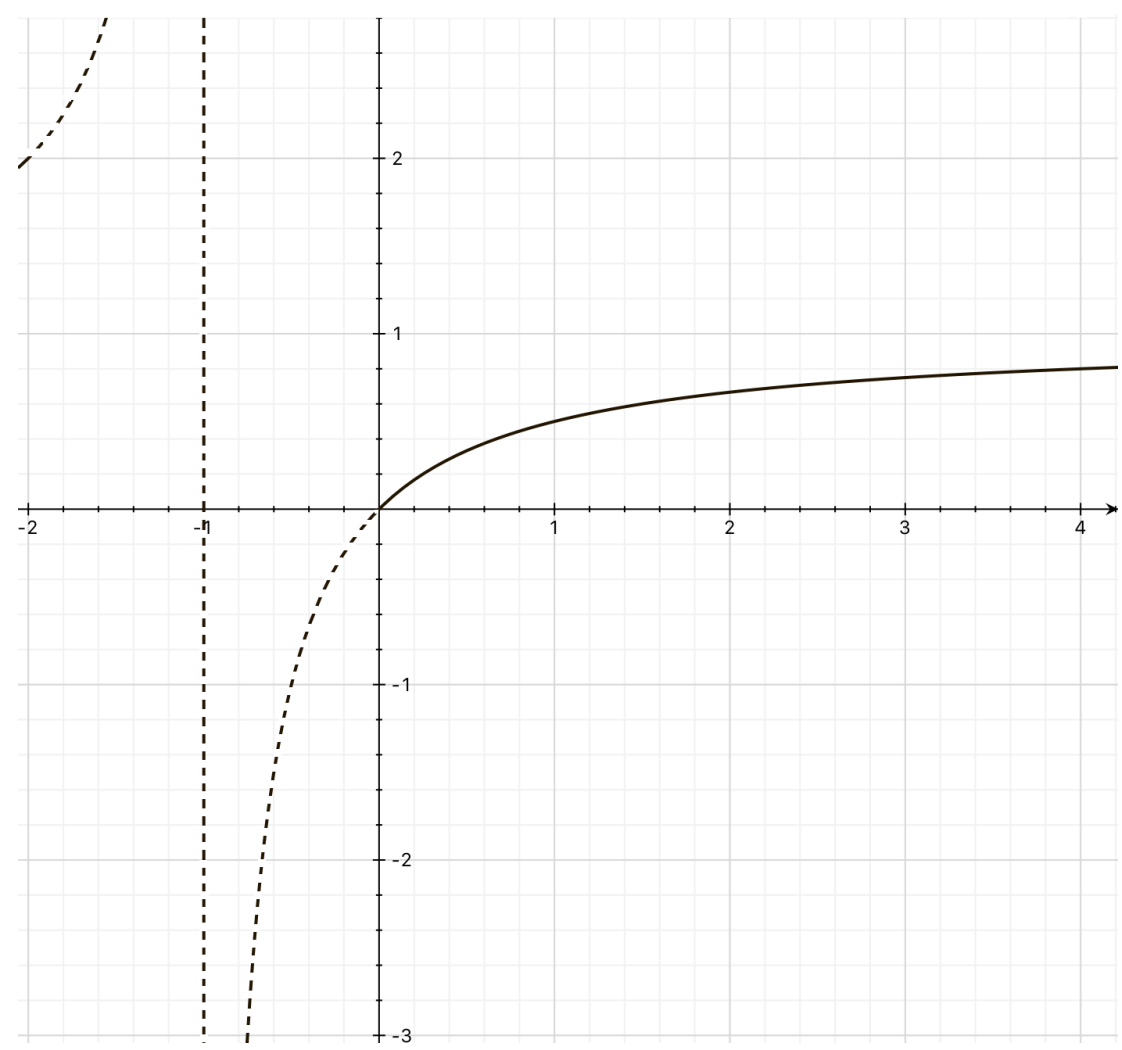

Figura 3.2: Gráfico de $h(s)=\frac{s}{s+1}$. 

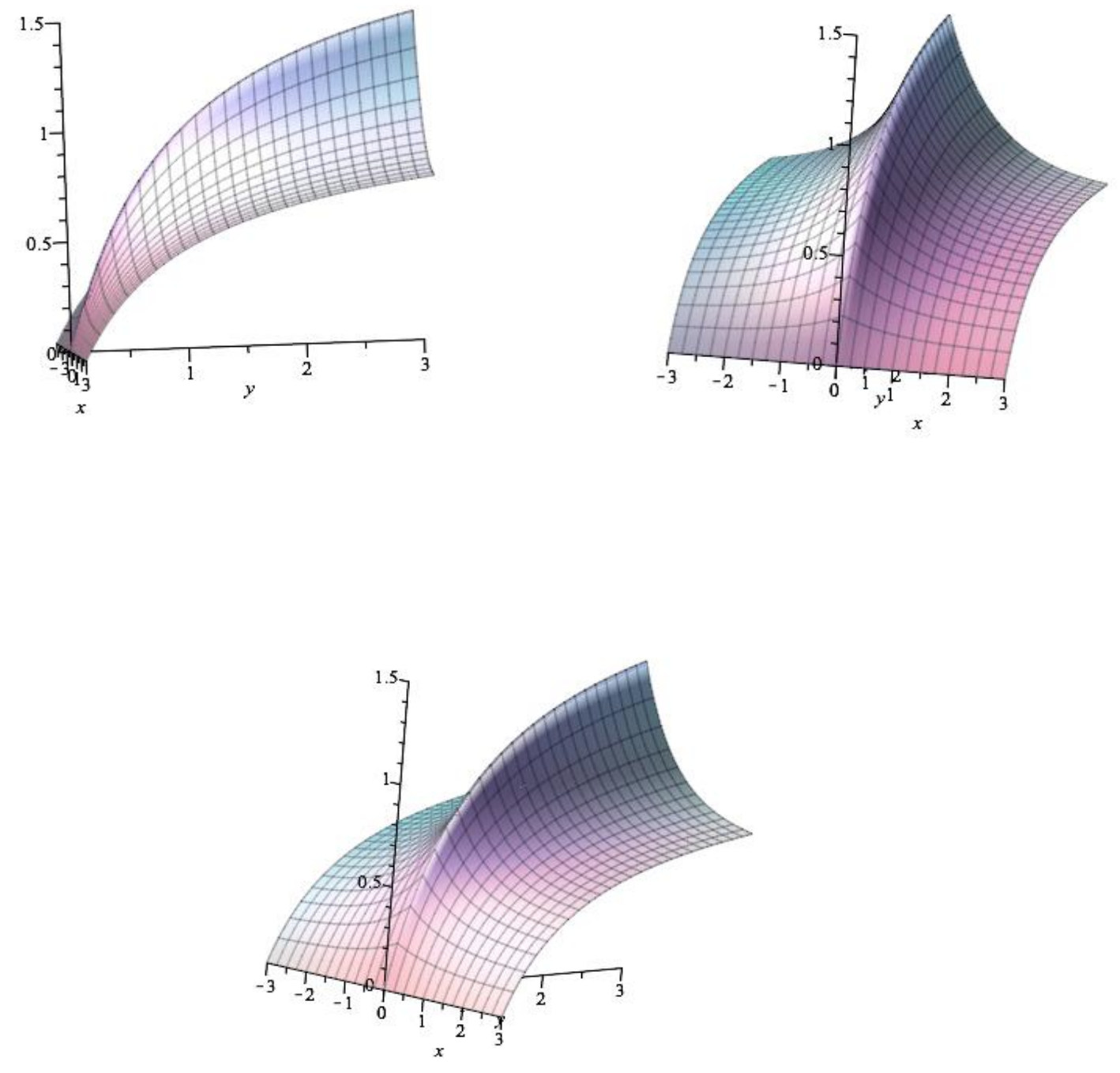

Figura 3.3: Gráfico de $f(x, s)=\left(\frac{e^{|x|}+1}{e^{|x|}}\right) \frac{s}{s+1}$. 


\section{Capítulo}

\section{Existência de Múltiplas Soluções}

Nesta seção, obtemos múltiplos resultados para o problema $\left(P_{\lambda}\right)$, sob uma condição extra de simetria.

\subsection{Simetria na não linearidade}

Vamos assumir que $f \in C\left(\mathbb{R}^{N} \times \mathbb{R}, \mathbb{R}^{+}\right)$satisfaz $\left(f_{1}\right)-\left(f_{4}\right)$ e

$$
\left(f_{6}\right) \quad f(x,-s)=f(x, s), \quad \forall x \in \mathbb{R}^{N}, s \in \mathbb{R}
$$

Então, segue que $h(s) \in C\left(\mathbb{R}, \mathbb{R}^{+}\right)$e $h(-s)=h(s)$, para todo $s \in \mathbb{R}$. Vamos encontrar soluções de $\left(P_{\lambda}\right)$ como pontos críticos do funcional energia $I_{\lambda}$ definido em $(2.1)$. Note que, por $\left(f_{6}\right), I_{\lambda}$ é agora um funcional par em $H^{1}\left(\mathbb{R}^{N}\right)$. De fato, primeiramente note que

$$
\begin{aligned}
F(x,-s) & =\int_{0}^{-s} f(x, t) t d t \\
& =-\int_{0}^{-s} f(x,-t)(-t) d t \\
& =\int_{0}^{s} f(x, \tau) \tau d \tau \\
& =F(x, s),
\end{aligned}
$$

onde fizemos a mudança $\tau=-t$. Assim, temos que

$$
\begin{aligned}
I_{\lambda}(-u) & =\frac{1}{2}\|-u\|_{\lambda}^{2}-\int F(x,-u) d x \\
& =\frac{1}{2}\|u\|_{\lambda}^{2}-\int F(x, u) d x \\
& =I_{\lambda}(u) .
\end{aligned}
$$


Agora, lembramos de um teorema de multiplicidade para funcionais pares que será usado na prova do nosso resultado de multiplicidade. Para isso precisamos da seguinte definição:

Definição 4.1. Seja $E$ um espaço de Banach real e denote por $\boldsymbol{X}$ a família de conjuntos $A \subset E \backslash\{0\}$ tais que $A$ é fechado em $E$ e simétrico com respeito a 0 , ou seja, $x \in A$ se, e somente se, $-x \in A$. Para $A \in \boldsymbol{X}$, defina o gênero de $A$ como $n$, denotado por $\gamma(A)=n$, se existe uma aplicação ímpar $\varphi \in C\left(A, \mathbb{R}^{n} \backslash\{0\}\right)$ onde $n$ é o menor inteiro positivo com essa propriedade.

Por exemplo, se considerarmos $B \subset E$ fechado com $B \cap(-B)=\varnothing$ e $A=B \cup(-B)$. Então $A \in \mathbf{X}$ e $\gamma(A)=1$, já que a função

$$
\varphi(x)=\left\{\begin{array}{lll}
1, & \text { para } B, \\
-1, & \text { para }(-B),
\end{array}\right.
$$

é ímpar e pertence a $C(A, \mathbb{R} \backslash\{0\})$.

Para nosso teorema de multiplicidade, seja $I \in C^{1}(E, \mathbb{R})$ um funcional par em um espaço de Banach de dimensão infinita E. Assuma que

(i) $I>0$, em $B_{\rho} \backslash\{0\}$ e $I \geq \alpha$, em $\partial B_{\rho}$, para algum $\alpha, \rho>0$;

(ii) Existe um subespaço k-dimensional $X_{k}$ de E, tal que

$$
X_{k} \cap A_{0} \text { é limitado e } \sup _{u \in X_{k}} I(u)=M<\infty
$$

onde

$$
A_{0}:=\{u \in E \mid I(u) \geq 0\}
$$

Denotando a bola unitária de $E$ por $B_{1}$ e $S_{1}$ sua fronteira, sejam

$$
\begin{aligned}
\Gamma & :=\left\{h \in C(E, E) \mid h \text { é um homeomorfismo ímpar, } h(0)=0, h\left(B_{1}\right) \subset A_{0}\right\} \quad \text { e } \\
\Gamma_{m} & :=\left\{K \subset E \mid K \text { compacto, }-K=K, \gamma\left(K \cap h\left(S_{1}\right)\right) \geq m, \forall h \in \Gamma\right\},
\end{aligned}
$$

onde $\gamma(K)$ é o gênero de um subconjunto simétrico $K \subset E$.

Teorema 4.1. Seja $E$ um espaço de Banach e $I \in C^{1}(E, \mathbb{R})$ satisfazendo as condições (i) e (ii) acima. Além disso assuma que I satisfaz $(C e)_{c}$, para todo $\alpha \leq c \leq M$. Seja

$$
b_{m}:=\inf _{K \in \Gamma_{m}} \sup _{u \in K} I(u), \quad m=1, \ldots, k
$$

Então

(1) $0<\alpha \leq b_{1} \leq \ldots \leq b_{k}<\infty$ e $b_{1}, \ldots, b_{k}$ são valores crítico de $I$;

(2) Se $b_{m}=b_{m+1}$ para algum $m \in\{1, \ldots, k\}$, então $I$ possui infinitos (pares de) pontos críticos correspondentes a $b_{m}$.

Quando o funcional $I$ satisfaz a condição de Palais-Smale $(P S)_{c}$, para $\alpha \leq c \leq M$, uma prova do Teorema 4.1 pode ser vista em Rabinowitz [16]. Contudo, a mesma prova funciona sob a condição, mais fraca, de Cerami $(\mathrm{Ce})_{c}$, com $\alpha \leq c \leq M$. Assim nossa tarefa nessa nova configuração é determinar os valores de $c>0$ para os quais $(C e)_{c}$ vale para nosso funcional $I_{\lambda}$. Na verdade, uma análise mais cuidadosa das provas do Lema 2.3, Lema 2.4, Proposição 2.1 e Teorema 2.2 nos mostram que, pelos mesmos argumentos usados nesses resultados, podemos provar o seguinte análogo ao Teorema 2.2: 
Teorema 4.2. Assuma $\left(f_{1}\right)-\left(f_{4}\right)$ e $\left(f_{6}\right)$. Se $0<\lambda<|\Lambda|$ e $\lambda \notin \sigma_{p}(S)$, então o funcional $I_{\lambda}$ satisfaz a condição de Cerami $(\mathrm{Ce})_{c}$, para todo $0<c<m_{\lambda}^{\infty}$.

Observação 4.1. Aqui, $\sigma_{p}(S)$ denota o espectral pontual do operador $S$ definido em (2.5). Em outras palavras,

$$
\sigma_{p}(S)=\left\{\lambda \in \mathbb{R} \mid-\Delta u-g(x) u=\lambda u, \text { para algum } 0 \neq u \in H^{2}\left(\mathbb{R}^{N}\right)\right\} .
$$

Observação 4.2. Os seguintes fatos são conhecidos sobre o espectro $\sigma(S)$ (veja [4]):

(a) $S$ tem um espectro discreto (não essencial) em $\left(-\infty,-l_{\infty}\right)$, ou seja, para qualquer $l>l_{\infty}$, o espectro de $S$ em $(-\infty,-l)$ consiste num número finito de autovalores de multiplicidade finita;

(b) Se $\lim _{|x| \rightarrow \infty}\left(g(x)-l_{\infty}\right)|x|=0$, então $\sigma_{p}(S) \cap\left(-l_{\infty}, \infty\right)=\varnothing$.

Note que, sob a hipótese $(b)$ acima, segue que $\sigma_{p}(S)$ é um subconjunto enumerável de $\left[\Lambda,-l_{\infty}\right]$.

Estamos agora prontos para enunciar e provar nosso resultado de multiplicidade.

\subsection{Resultado de Multiplicidade}

Teorema 4.3. Assuma as condições $\left(f_{1}\right)-\left(f_{4}\right),\left(f_{6}\right)$ e $0<\lambda<|\Lambda|, \lambda \notin \sigma_{p}(S)$. Assuma também que existem $k$ funções de suporte disjuntos $\phi_{1}, \ldots, \phi_{k} \in H^{1}\left(\mathbb{R}^{N}\right)$, tais que

$$
\begin{gathered}
I_{\lambda}\left(\phi_{i}\right)<0 \\
\left\|\phi_{i}\right\|_{\lambda}^{2}<\frac{2 m_{\lambda}^{\infty}}{k},
\end{gathered}
$$

para todo $1 \leq i \leq k$, onde $I_{\lambda}$ é o funcional energia definido em (2.1), ou seja,

$$
I_{\lambda}(u)=\frac{1}{2}\|u\|_{\lambda}^{2}-\int F(x, u) d x, \quad u \in H^{1}\left(\mathbb{R}^{N}\right) .
$$

Então o problema $\left(P_{\lambda}\right)$ tem pelo menos $k$ pares de soluções não triviais.

Demonstração. Vamos aplicar o Teorema 4.1 em $I_{\lambda} \operatorname{com} E=H^{1}\left(\mathbb{R}^{N}\right)$. Primeiramente, a Proposição 3.1 implica que a condição (i) do Teorema 4.1 é satisfeita por $I_{\lambda}$. Em seguida, definimos

$$
X_{k}:=\operatorname{span}\left\{\phi_{i} \mid 1 \leq i \leq k\right\} \subset H^{1}\left(\mathbb{R}^{N}\right)
$$

e mostraremos que $\sup _{u \in X_{k}} I_{\lambda}(u)<m_{\lambda}^{\infty}$. Como as funções $\phi_{i}$ possuem suportes disjuntos, temos que

$$
I_{\lambda}\left(\sum_{i=1}^{k} t_{i} \phi_{i}\right)=\sum_{i=1}^{k} I_{\lambda}\left(t_{i} \phi_{i}\right)
$$

onde

$$
I_{\lambda}\left(t_{i} \phi_{i}\right)=\frac{1}{2} t_{i}^{2}\left\|\phi_{i}\right\|_{\lambda}^{2}-\int F\left(x, t_{i} \phi_{i}\right) d x .
$$

De fato, note que $\phi_{i} \phi_{j}=0$, para $i \neq j$, já que os suportes são disjuntos. Em particular, note que

$$
\begin{aligned}
\int\left(\phi_{i}+\phi_{j}\right)^{2} d x & =\int\left(\phi_{i}^{2}+2 \phi_{i} \phi_{j}+\phi_{j}^{2}\right) d x \\
& =\int \phi_{i}^{2} d x+\int \phi_{j}^{2} d x
\end{aligned}
$$


logo, concluímos que

$$
\int\left(\sum_{i=1}^{k} t_{i} \phi_{i}\right)^{2} d x=\sum_{i=1}^{k} \int\left(t_{i} \phi_{i}\right)^{2} d x
$$

e com o mesmo argumento, temos

$$
\int\left|\nabla\left(\sum_{i=1}^{k} t_{i} \phi_{i}\right)\right|^{2} d x=\sum_{i=1}^{k} \int\left|\nabla t_{i} \phi_{i}\right|^{2} d x
$$

desta forma, obtemos

$$
\left\|\sum_{i=1}^{k} t_{i} \phi_{i}\right\|_{\lambda}^{2}=\sum_{i=1}^{k}\left\|t_{i} \phi\right\|_{\lambda}^{2} .
$$

Seja agora $x \in \operatorname{supp}\left\{\phi_{i}\right\}, \operatorname{logo} F\left(x, \phi_{j}\right)=F(x, 0)=0$, para $j \neq i$. Assim temos, para $x \in \operatorname{supp}\left\{\phi_{i}\right\}$, que

$$
F\left(x, \sum_{i=1}^{k} t_{i} \phi_{i}\right)=F\left(x, t_{i} \phi_{i}\right)=\sum_{i=1}^{k} F\left(x, t_{i} \phi_{i}\right),
$$

e $\operatorname{assim}(4.4)$ e (4.5) provam (4.3).

Agora estudaremos as seguintes possibilidades:

(1) $\left|t_{i}\right| \leq 1$ : Neste caso, note que de $\left(f_{1}\right)$ sabemos que $f(x, t) \geq 0, \forall t \in \mathbb{R}$, logo para $t \geq 0$, temos

$$
F(x, s)=\int_{0}^{s} f(x, t) t d t \geq 0
$$

para $s \geq 0$ e por $\left(f_{6}\right)$ temos que $F(x,-s)=F(x, s) \geq 0$, para todo $x \in \mathbb{R}^{N}$ e $s \in \mathbb{R}$. Teremos então

$$
\begin{array}{rlrl}
I_{\lambda}\left(t_{i} \phi_{i}\right) & =\frac{1}{2} t_{i}^{2}\left\|\phi_{i}\right\|_{\lambda}^{2}-\int F\left(x, t_{i} \phi_{i}\right) d x \\
& \leq \frac{1}{2} t_{i}^{2}\left\|\phi_{i}\right\|_{\lambda}^{2}, & & \text { pois } F(x, s) \geq 0 \\
& \leq \frac{1}{2}\left\|\phi_{i}\right\|_{\lambda}^{2}, & & \text { pois }\left|t_{i}\right| \leq 1 \\
& <\frac{m_{\lambda}^{\infty}}{k}, & & \text { por }(4.2),
\end{array}
$$

ou seja,

$$
I_{\lambda}\left(t_{i} \phi_{i}\right)<\frac{m_{\lambda}^{\infty}}{k}
$$

(2) $\left|t_{i}\right|>1$ : Neste caso, de $\left(f_{2}\right)$, obtemos

$$
\begin{aligned}
F(x, t s) & =\int_{0}^{t s} f(x, \tau) \tau d \tau \\
& =\int_{0}^{s} f(x, t \alpha)(t \alpha) t d \alpha \\
& =t^{2} \int_{0}^{s} f(x, t \alpha) \alpha d \alpha \\
& \geq t^{2} \int_{0}^{s} f(x, \alpha) \alpha d \alpha \\
& =t^{2} F(x, s)
\end{aligned}
$$


para $|t| \geq 1$ e $s \geq 0$, onde fizemos a mudança $\alpha=\frac{\tau}{t}$. Por $\left(f_{6}\right)$ e (4.7), temos

$$
F(x,-t s)=F(x, t s) \geq t^{2} F(x, s)=t^{2} F(x,-s),
$$

e assim concluímos que

$$
F(x, t s) \geq t^{2} F(x, s)
$$

para $|t| \geq 1$ e $\forall s \in \mathbb{R}$. Obtemos então

$$
\begin{array}{rlrl}
I_{\lambda}\left(t_{i} \phi_{i}\right) & =\frac{1}{2} t_{i}^{2}\left\|\phi_{i}\right\|_{\lambda}^{2}-\int F\left(x, t_{i} \phi_{i}\right) d x & \\
& \leq t_{i}^{2}\left[\frac{1}{2}\left\|\phi_{i}\right\|_{\lambda}^{2}-\int F\left(x, \phi_{i}\right) d x\right], & & \text { por }(4.8) \\
& =t_{i}^{2} I_{\lambda}\left(\phi_{i}\right)<0, & & \text { por }(4.1)
\end{array}
$$

ou seja,

$$
I_{\lambda}\left(t_{i} \phi_{i}\right) \leq t_{i}^{2} I_{\lambda}\left(\phi_{i}\right)<0
$$

Portanto (4.6) e (4.9) nos dá

$$
\sup _{t_{i} \in \mathbb{R}} I_{\lambda}\left(t_{i} \phi_{i}\right)<\frac{m_{\lambda}^{\infty}}{k} \quad \text { e } \quad \lim _{|t| \rightarrow \infty} I_{\lambda}\left(t \phi_{i}\right)=-\infty, \quad 1 \leq i \leq k .
$$

Seja $u \in X_{k}$, logo $u=\sum_{i=1}^{k} t_{i} \phi_{i}$ e assim

$$
\begin{array}{rlrl}
\sup _{u \in X_{k}} I_{\lambda}(u) & =\sup _{\left(t_{1}, \ldots, t_{k}\right) \in \mathbb{R}^{k}} I_{\lambda}\left(\sum_{i=1}^{k} t_{i} \phi_{i}\right) & \\
& =\sum_{i=1}^{k} \sup _{\left(t_{1}, \ldots, t_{k}\right) \in \mathbb{R}^{k}} I_{\lambda}\left(t_{i} \phi_{i}\right), & & \text { por }(4.3) \\
& <k \cdot \frac{m_{\lambda}^{\infty}}{k}=m_{\lambda}^{\infty}, & &
\end{array}
$$

ou seja,

$$
\sup _{u \in X_{k}} I_{\lambda}(u)<m_{\lambda}^{\infty}
$$

Assim, fica claro de (4.10) e (4.11) que a condição (ii) do Teorema 4.1 também é satisfeita pela nossa escolha de $X_{k}$. Finalmente, o Teorema 4.2 fornece a condição de Cerami necessária, de modo que o Teorema 4.1 pode ser aplicado para obtermos o resultado de multiplicidade desejado.

Em seguida, vamos apresentar uma grande classe de tais problemas assintoticamente lineares possuindo múltiplas soluções.

Sejam $a(x) \in C\left(\mathbb{R}^{N}, \mathbb{R}^{+}\right), b(x) \in C\left(\mathbb{R}^{N}, \mathbb{R}^{+}\right)$e $p(s) \in C\left(\mathbb{R}, \mathbb{R}^{+}\right)$funções satisfazendo as seguintes condições:

$\left(h_{1}\right)$ Existe $a_{\infty}>0$ tal que $\lim _{|x| \rightarrow \infty}|x|\left(a(x)-a_{\infty}\right)=0$. Note que neste caso $\lim _{|x| \rightarrow \infty} a(x)=a_{\infty} ;$

$\left(h_{2}\right) b(x) \neq 0$ e $\lim _{|x| \rightarrow \infty}|x| b(x)=0$. Neste caso, $\lim _{|x| \rightarrow \infty} b(x)=0$; 
$\left(h_{3}\right) p(s)$ é uma função par, não-decrescente para $0 \leq s<\infty$ e satisfaz as três seguintes condições

$$
\begin{array}{rlrl}
p(s) & >0, & \text { para } s \neq 0, \\
\lim _{s \rightarrow 0} p(s) & =0 \quad \mathrm{e} \\
\lim _{|s| \rightarrow \infty} p(s) & =p_{\infty}>0 .
\end{array}
$$

Definimos agora

$$
f_{\mu}(x, s)=(a(x)+\mu b(x)) p(s), \quad \mu>0
$$

e consideramos o problema

$$
\left(\hat{P}_{\mu}\right) \quad-\Delta u+\lambda u=f_{\mu}(x, u) u .
$$

Note que, em vista de $\left(h_{1}\right)-\left(h_{3}\right)$, a função $f_{\mu}(x, s)$ satisfaz todas as condições $\left(f_{1}\right)-\left(f_{4}\right)$ e $\left(f_{6}\right)$. De fato,

$\left(f_{1}\right)$ : Como $a(x), b(x)$ e $p(s)$ são contínuas, devemos ter $f_{\mu} \in C\left(\mathbb{R}^{N} \times \mathbb{R}, \mathbb{R}^{+}\right)$e

$$
\lim _{s \rightarrow 0} f_{\mu}(x, s)=(a(x)+\mu b(x)) \lim _{s \rightarrow 0} p(s)=0, \quad \text { uniformemente em } x ;
$$

$\left(f_{2}\right)$ : Para $x \in \mathbb{R}^{N}$ fixo temos, por $\left(h_{3}\right)$, que $f(x, \cdot)$ é não-decrescente para $s \in[0, \infty)$. Definindo $g(x):=g_{\mu}(x)=p_{\infty}(a(x)+\mu b(x))$, teremos $g \in C\left(\mathbb{R}^{N}, \mathbb{R}^{+}\right) \mathrm{e}$

$$
\begin{aligned}
\lim _{s \rightarrow \infty} f(x, s) & =(a(x)+\mu b(x)) \lim _{s \rightarrow \infty} p(s) \\
& =p_{\infty}(a(x)+\mu b(x)) \\
& =g(x)
\end{aligned}
$$

uniformemente em $x$.

$\left(f_{3}\right)$ : Seja $h(s)=p(s) a_{\infty}, \operatorname{logo} h \in C\left(\mathbb{R}, \mathbb{R}^{+}\right) \mathrm{e}$

$$
\begin{aligned}
\lim _{|x| \rightarrow \infty} f(x, s) & =p(s) \lim _{|x| \rightarrow \infty}(a(x)+\mu b(x)) \\
& =p(s) a_{\infty} \\
& =h(s),
\end{aligned}
$$

uniformemente em $s$.

$\left(f_{4}\right)$ : Temos

$$
\begin{aligned}
\lim _{|x|, s \rightarrow \infty} f(x, s) & =\lim _{|x| \rightarrow \infty}(a(x)+\mu b(x)) \lim _{s \rightarrow \infty} p(s) \\
& =a_{\infty} p_{\infty} \\
& :=l_{\infty} \in(0, \infty) .
\end{aligned}
$$


$\left(f_{6}\right)$ : Como $p(s)$ é uma função par, então

$$
\begin{aligned}
f(x,-s) & =(a(x)+\mu b(x)) p(-s) \\
& =(a(x)+\mu b(x)) p(s) \\
& =f(x, s) .
\end{aligned}
$$

Lembramos agora das definições dadas em $(2.23)$ - (2.25), são elas:

$$
\begin{gathered}
I_{\lambda}^{\infty}(u)=\frac{1}{2}\|u\|_{\lambda}^{2}-\int H(u) d x, \\
M_{\lambda}^{\infty}=\left\{u \neq 0 \mid I_{\lambda}^{\infty \prime}(u) u=\|u\|_{\lambda}^{2}-\int h(u) u d x=0\right\}, \\
0<m_{\lambda}^{\infty}=\inf _{u \in M_{\lambda}^{\infty}} I_{\lambda}^{\infty}(u), \quad \text { se } M_{\lambda}^{\infty} \neq \varnothing \quad \text { e } \\
m_{\lambda}^{\infty}=\infty, \quad \text { se } M_{\lambda}^{\infty} .
\end{gathered}
$$

Já que $\lim _{|x| \rightarrow \infty} f_{\mu}(x, s)=h(s)$ é independente de $\mu$, então podemos ver que $m_{\lambda}^{\infty}$ é independente de $\mu$. Além disso, como $\left(h_{1}\right)$ e $\left(h_{2}\right)$ implicam que

$$
\begin{aligned}
|x|\left(g_{\mu}(x)-a_{\infty} p_{\infty}\right) & =|x|\left(p_{\infty}(a(x)+\mu b(x))-a_{\infty} p_{\infty}\right) \\
& =|x|\left(a(x) p_{\infty}+\mu b(x) p_{\infty}-a_{\infty} p_{\infty}\right) \\
& =|x|\left(\left(a(x)-a_{\infty}\right) p_{\infty}+a_{\infty} p_{\infty}+\mu b(x) p_{\infty}-a_{\infty} p_{\infty}\right) \\
& =|x|\left(a(x)-a_{\infty}\right) p_{\infty}+\mu p_{\infty}|x| b(x) \rightarrow 0,
\end{aligned}
$$

quando $|x| \rightarrow \infty$, temos então que

$$
\lim _{|x| \rightarrow \infty}|x|\left(g_{\mu}(x)-l_{\infty}\right)=0, \quad \text { para todo } \mu>0 .
$$

Concluímos da Observação 4.2 que, se $0<\lambda<l_{\infty}=a_{\infty} p_{\infty}$, então $\lambda \notin \sigma_{p}\left(S_{\mu}\right)$ para todo $\mu>0$, onde

$$
S_{\mu}: H^{2}\left(\mathbb{R}^{N}\right) \subset L^{2}\left(\mathbb{R}^{N}\right) \rightarrow L^{2}\left(\mathbb{R}^{N}\right) \text { e } S_{\mu}(u):=-\Delta u-g_{\mu}(x) u .
$$

Agora, dado $k \in \mathbb{N}$ podemos escolher $k$ funções de suporte disjuntos $0 \leq \phi_{1}, \ldots, \phi_{k} \in C_{0}^{\infty}\left(\mathbb{R}^{N}\right)$ tais que, para $1 \leq i \leq k$,

$$
\left\|\phi_{i}\right\|_{\lambda}^{2}<\frac{2 m_{\lambda}^{\infty}}{k} \quad \text { e } \quad \int b(x) P\left(\phi_{i}\right) d x>0
$$


onde $P(s)=\int_{0}^{s} p(t) t d t$. Note que

$$
\begin{aligned}
F_{\mu}(x, s) & =\int_{0}^{s} f_{\mu}(x, t) t d t \\
& =\int_{0}^{s}(a(x)+\mu b(x)) p(t) t d t \\
& =(a(x)+\mu b(x)) \int_{0}^{s} p(t) t d t \\
& =(a(x)+\mu b(x)) P(s) .
\end{aligned}
$$

Então, como $a(x) \geq 0$, segue que

$$
\begin{aligned}
I_{\lambda}\left(\phi_{i}\right) & =\frac{1}{2}\left\|\phi_{i}\right\|_{\lambda}^{2}-\int F_{\mu}\left(x, \phi_{i}\right) d x \\
& =\frac{1}{2}\left\|\phi_{i}\right\|_{\lambda}^{2}-\int(a(x)+\mu b(x)) P\left(\phi_{i}\right) d x \\
& \leq \frac{1}{2}\left\|\phi_{i}\right\|_{\lambda}^{2}-\mu \int b(x) P\left(\phi_{i}\right) d x
\end{aligned}
$$

de modo que, para $\mu>0$ suficientemente grande, tenhamos $I_{\lambda}\left(\phi_{i}\right)<0$, para $1 \leq i \leq k$. Provamos o seguinte resultado.

Teorema 4.4. Assuma que $a(x) \in C\left(\mathbb{R}^{N}, \mathbb{R}^{+}\right), b(x) \in C\left(\mathbb{R}^{N}, \mathbb{R}^{+}\right)$e $p(s) \in C\left(\mathbb{R}, \mathbb{R}^{+}\right)$satisfazem as condições $\left(h_{1}\right)-\left(h_{3}\right)$. Então, para qualquer $0<\lambda<a_{\infty} p_{\infty}$, o número de soluções do problema $\left(\hat{P}_{\mu}\right)$ tende ao infinito quando $\mu \rightarrow \infty$. 


\section{Referências Bibliográficas}

[1] Adams, R., Sobolev Spaces, Academic Press, 1975.

[2] Ambrosetti, A. and Rabinowitz, P. H., Dual variational methods in critical point theory and applications, J. Functional Analysis 14 (1973), 349-381.

[3] Bartle, R. G., The Elements of Integration, New York - London - Sydney, 1966.

[4] Berezin, F. A. e Shubin, M. A., The Schrodinger Equation, Kluwer, Dordrecht, 1991.

[5] Brezis, H., Functional Analysis, Sobolev Spaces and Partial Differential Equations, Springer, Rutgers University, 2010.

[6] Costa, D. G. e Tehrani, H., On a Class of Assimptotically Linear Elliptic Problems in $\mathbb{R}^{N}$, Journal of Differential Equations 173 (2001), 470-494.

[7] Dautray, R. e Lions, J.L., Analyse Mathématique et Calcul Numérique pour les Sciences et les Techniques, Vol 1, Masson, Paris, 1984.

[8] Evans, L. C., Partial Differential Equations, American Math. Soc., 1998.

[9] Figueiredo, G., Uma Introdução à Teoria dos Pontos Críticos, Universidade Federal do Pará, 2015.

[10] Furtado, M., Notas de EDP 2, versão 1.2, Universidade de Brasília, Brasília, 2012.

[11] Gilbarg, D. e Trudinger, N. S., Elliptic Partial Differential Equations of Second Order, Springer, Berlin-New York, 1983.

[12] Kavian, O., Introduction à la Théorie des Points Critiques, Springer-Verlag, France, Paris, 1993.

[13] Kreyszig, E., Introductory Functional Analysis with Applications, Wiley Classics Library, University of Windsor, 1978.

[14] Lions, P. L., The Concentration-Compactness Principle in the Calculus of Variations. The Locally Compact Case, Non Linéaire 1, 1984, nº 2, 109-145.

[15] Oliveira, C. R. de, Intermediate Spectral Theory and Quantum Dynamics, Birkhäuser, Progress in Mathematical Physics, volume 54, 2009. 
[16] Rabinowitz, P. H., Minimax Methods in Critical Point Theory with Applications to Differential Equations, CBMS Regional Conf. Ser. in Math, Vol. 65, Amer. Math. Soc. Providence, RI, 1986.

[17] Stein, E., Singular Integrals and Differentiability Properties of Functions, Princeton Univ. Press, Princieton, NJ, 1970.

[18] Stuart, C.A. e Zhou, H.S., Applying the mountain pass theorem to an asymptotically linear elliptic equation on $\mathbb{R}^{N}$, Comm. Partial Differential Equations 24 (1999), 1731-1758.

[19] Willem, M., Minimax Theorems, Birkhäuser, Boston, 1996. 